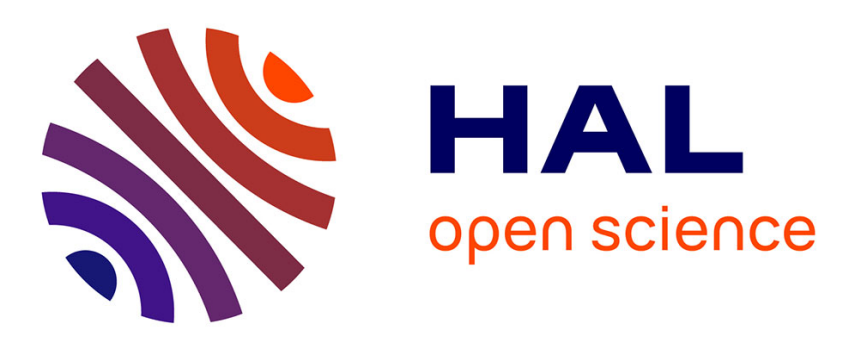

\title{
Numerical characterization and computation of dynamic instabilities for frictional contact problems
}

\author{
Michel Raous, Serge Barbarin, Didier Vola
}

\section{To cite this version:}

Michel Raous, Serge Barbarin, Didier Vola. Numerical characterization and computation of dynamic instabilities for frictional contact problems. J.A.C. Martins and M. Raous. CISM Course: Friction and instabilities, Coll. CISM Courses and Lectures n457, Springer Verlag, pp.233-292, 2002, 457. hal-00166137

\section{HAL Id: hal-00166137 https://hal.science/hal-00166137}

Submitted on 3 Dec 2020

HAL is a multi-disciplinary open access archive for the deposit and dissemination of scientific research documents, whether they are published or not. The documents may come from teaching and research institutions in France or abroad, or from public or private research centers.
L'archive ouverte pluridisciplinaire HAL, est destinée au dépôt et à la diffusion de documents scientifiques de niveau recherche, publiés ou non, émanant des établissements d'enseignement et de recherche français ou étrangers, des laboratoires publics ou privés. 
Reference :

M. RAOUS, S. BARBARIN, D.VOLA, Numerical characterization and computation of dynamic instabilities for frictional contact problems, in "Friction and instabilities", J.A.C. Martins- M. Raous (Eds), CISM Courses and Lectures, $n$ 457, Springer Verlag, Wien-New York, 2002, pp.233-292. 


\title{
Numerical characterization and computation of dynamic instabilities for frictional contact problems
}

\author{
Michel Raous, Serge Barbarin, Didier Vola ${ }^{\star}$ \\ Laboratoire de Mécanique et d'Acoustique, CNRS, Marseille, France
}

\begin{abstract}
This chapter focuses on the numerical aspects of the characterization of frictioninduced instabilities and their dynamic computation for linear and nonlinear problems. We begin by presenting briefly basic formulations and several computational methods for solving unilateral frictional contact problems, in quasi-statics and dynamics, and in elasticity and hyper-elasticity. The above specific dynamic formulations will be used to compute the flutter solutions presented in the last sections. Numerical schemes are then given for computing the various sufficient or necessary conditions for instability established together with Professor J.A.C. Martins. Finally, the stability analysis and the computation of flutter solutions are carried out for two examples : the sliding of a Polyurethane block on a plane and the squeal of a rubber waist seal sliding on a car window.

In Section 1, a variational inequality formulation and numerical methods for solving quasistatic problems in elasticity are briefly recalled. Details can be found in a previous CISM course volume (see Raous (1999)).

This approach is extended to dynamic problems in Section 2 . The formulation is written in terms of differential measures in order to deal with the non-smooth character of the solutions. It is an extension of those developed by J.J. Moreau and M. Jean.

In Section 3, the above formulations are extended to hyper-elastic problems and a method for computing directly the steady sliding solution is given.

Numerical analysis of the stability of quasistatic solutions in the context of linear elasticity is carried out in Section 4. The example of the sliding of a Polyurethane block is studied.

In Section 5, the stability analysis is carried out for a steady sliding solution in the context of hyper-elasticity and used to characterize the squeal of a waist seal sliding on a car window.
\end{abstract}

\footnotetext{
^ This work has been conducted within an International Program of Scientific Collaboration (PICS) between CNRS and JNICT with Professor J.A.C. Martins (IST - Lisbon) involving the theses of S. Barbarin, D. Vola in Marseille and the one of A. Pinto da Costa in Lisbon. The study on the waist seal presented in sections 3 and 5 has been supported by Renault.
} 


\section{Formulation and numerical methods for solving quasistatic frictional unilateral problems in small deformations}

In this section, we briefly recall the formulation of unilateral contact problems involving Coulomb friction. The variational formulation is given in terms of implicit variational inequalities and various solvers are presented. Details can be found in Raous (1999), where an extensive bibliography is also given.

\subsection{The Signorini problem with Coulomb friction}

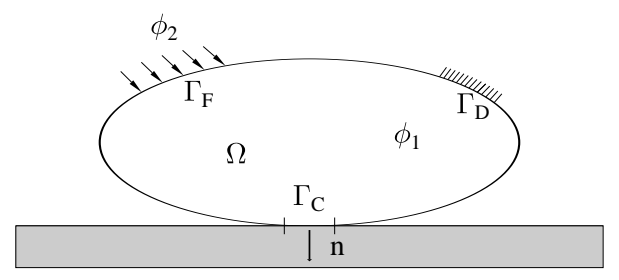

Figure 1. Contact with a rigid obstacle.

\section{a - Unilateral conditions (Signorini problem)}

The displacements and the stresses exerted on the part of the boundary where contact is expected to occur are written as follows, where $n$ denotes the outwards normal to the solid :

$$
\begin{aligned}
& u=u_{N} n+u_{T}, \\
& r=r_{N} n+r_{T} .
\end{aligned}
$$

The unilateral conditions are then written :

$$
\left.\begin{array}{l}
u_{N} \leq 0, \\
r_{N} \leq 0, \\
u_{N} r_{N}=0 .
\end{array}\right\}
$$

We avoid the use of compliance models and penalty techniques (for the computations) in order to obtain solutions which do not depend on mechanical or numerical parameters which may influence the stability analysis. This is then a non smooth model (multivalued application).

\section{b - The Coulomb friction}

$$
\left.\begin{array}{l}
\left\|r_{T}\right\| \leq \mu\left|r_{N}\right| \text { with : } \\
\text { if }\left\|r_{T}\right\|<\mu\left|r_{N}\right| \text { then } \dot{u}_{T}=0, \\
\text { if }\left\|r_{T}\right\|=\mu\left|r_{N}\right| \text { then } \dot{u}_{T} \text { is colinear and opposite to } r_{T} .
\end{array}\right\}
$$

It should be noted that : 
- this law is not associated (no normality rule for the sliding direction),

- it is a velocity formulation whereas the unilateral contact has displacement formulation,

- the graph is also a multivalued application (see Fig. 2).

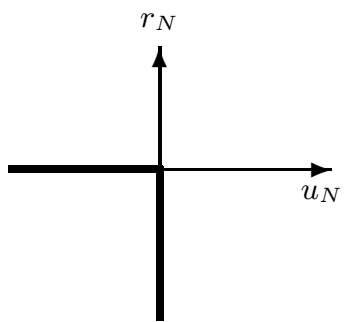

Unilateral contact

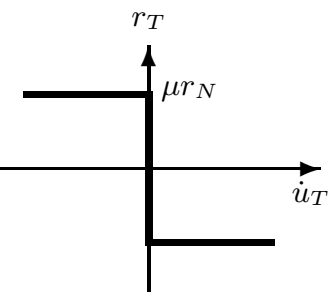

Coulomb friction

Figure 2. Graphs of the contact and of the friction law.

\section{c - The problem}

Problem $\left(\boldsymbol{P}_{\boldsymbol{c}}\right)$ : Let $\phi_{1}, \phi_{2}$ be the given forces, find the displacement $u$, the strain $\varepsilon$, the stress $\sigma$ and the contact stress $r$ such that :

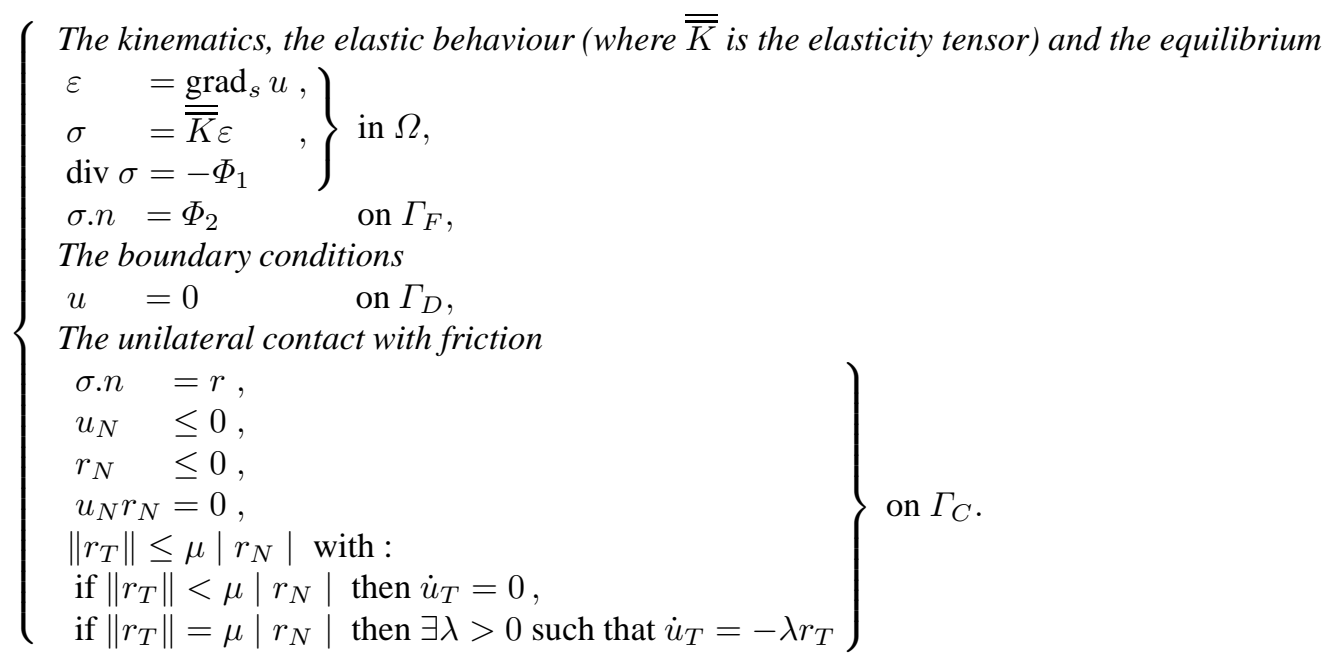

In what follows, the "static" frictional problem will be obtained by replacing $\dot{u}$ by $u$ in Problem $\left(\boldsymbol{P}_{\boldsymbol{c}}\right)$. This makes sense only in some very specific cases, but it will be helpful when used as an intermediate problem with some solvers.

\subsection{Various formulations}

Various types of formulation are briefly outlined below (see also Raous (2001)). The first two types will be used in the present study. 


\section{a - Variational inequalities, fixed point and minimization under constraints}

Cocu-Pratt-Raous (1996) have shown that, by eliminating the contact stresses, a system of two variational inequalities (one of which is implicit) can be obtained (see Problem $(P)$ ). After performing a time discretization $\left(t^{i+1}=t^{i}+\Delta t\right)$, an incremental form $\left(\boldsymbol{P}_{\boldsymbol{i n c 1} 1}\right)$ is obtained and the problem is reduced to a single implicit variational inequality $\left(\boldsymbol{P}_{\boldsymbol{i n c 2} 2}\right)$. At each time step, we have to solve a "static" problem including extra terms depending on the previous history of the solution. By using a fixed point method on the sliding limit, the problem is set as a sequence of problems consisting in a minimization under constraints $\left(\boldsymbol{P}_{\boldsymbol{o p t} \mathbf{1}}\right)$.

The variational problem $(\boldsymbol{P})$ : Find $u(t) \in \mathcal{K}$ such that :

$$
\left\{\begin{array}{c}
a(u(t), v-\dot{u}(t))+J_{1}(u(t), v)-J_{1}(u(t), \dot{u}(t)) \geq L(v-\dot{u}(t)) \\
+<r_{N}(u(t)), v_{N}-\dot{u}_{N}(t)>\quad \forall v \in \mathcal{V}, \\
\left.<r_{N}(u(t)), z_{N}-u_{N}(t)\right)>\geq 0 \quad \forall z \in \mathcal{K} .
\end{array}\right.
$$

where :

- $a(u, v)$ is a bilinear form associated with the elasticity mapping,

- $J_{1}(u, v)=\int_{\Gamma_{C}}-\mu r_{N}(u)\left\|v_{T}\right\| d s$,

- $\mathcal{K}=\left\{v \in \mathcal{U} / v_{N} \leq 0\right.$ on $\left.\Gamma_{C}\right\}$,

- $L(v)$ is a linear form associated with the loading $\phi_{1}$ and $\phi_{2}$,

The incremental problem $\left(\boldsymbol{P}_{\text {inc1 } 1}\right)$ : Find $u^{i+1} \in \mathcal{K}$ such that :

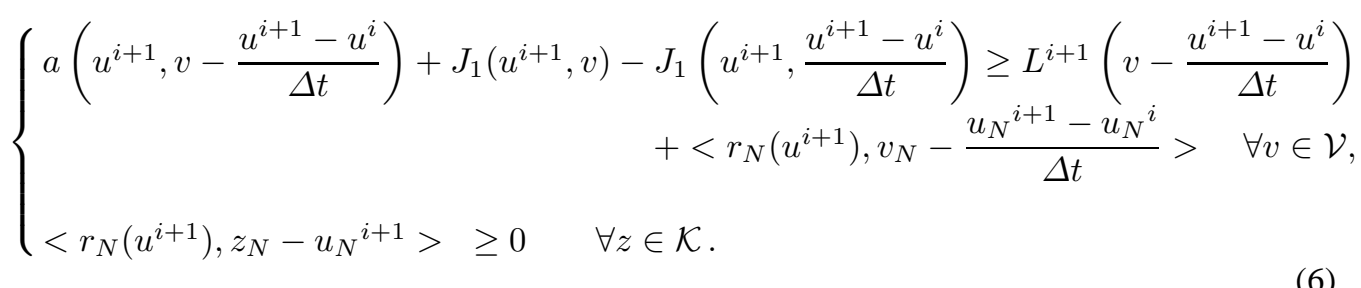

Equivalence with the following problem has been established (see Cocu-Pratt-Raous (1996)) :

The reduced incremental problem $\left(\boldsymbol{P}_{\text {inc2 } 2}\right)$ : Find $u^{i+1} \in \mathcal{K}$ such that :

$$
a\left(u^{i+1}, w-u^{i+1}\right)+J_{1}\left(u^{i+1}, w-u^{i}\right)-J_{1}\left(u^{i+1}, u^{i+1}-u^{i}\right) \geq L^{i+1}\left(w-u^{i+1}\right) \forall w \in \mathcal{K} .
$$

A fixed point method is introduced on the sliding threshold $g$ in order to ensure that : $g=-\mu r_{N}$. At every fixed point iteration, a friction problem with a given threshold $g$ (Tresca friction problem) has to be solved. In this case, the problem can be written as a minimization one. Either problem $\left(\boldsymbol{P}_{\text {opt1 } 1}\right)$ (set on the displacements) or problem $\left(\boldsymbol{P}_{\text {opt } 2}\right)$ (written on the displacement increments) can be obtained :

The minimization problem $\left(\boldsymbol{P}_{\text {opt } 1}\right)$ : For a given $g$, find $u_{g}{ }^{i+1} \in \mathcal{K}$ such that

$$
J\left(u_{g}{ }^{i+1}\right) \leq J(v) \quad \forall v \in \mathcal{K},
$$

with:

$$
J(v)=\frac{1}{2} a(v, v)+j\left(v-u^{i}\right)-L^{i+1}(v),
$$


$-j(v)=\int_{\Gamma_{C}} g\left\|v_{T}\right\| d s$,

- the convex does not change,

- the term $u^{i}$ contains the memory of the loading path.

The minimization problem $\left(\boldsymbol{P}_{\text {opt } 2}\right)$ : For a given $g$, find $\Delta u_{g}{ }^{i+1} \in \mathcal{K}^{i}$ such that

$$
J\left(\Delta u_{g}{ }^{i+1}\right) \leq J(v) \quad \forall v \in \mathcal{K}^{i},
$$

with :

$$
J(v)=\frac{1}{2} a(v, v)+j(v)-\Delta L^{i+1}(v)-r^{i}(v),
$$

- the convex depends on the previous step : $\mathcal{K}^{i}=\left\{v \in \mathcal{U} / u_{i}+v_{N} \leq 0\right.$ on $\left.\Gamma_{C}\right\}$,

- the memory of the loading path is in $r^{i}$ (contact stress of the previous step computed as the defect of equilibrium).

\section{b - A complementarity problem (discretized form)}

The formulation is here given for a static problem after finite element discretization (in the following, vectors of $\mathbb{R}^{n}$ associated to the discretization are denoted by bold characters). It can be extended to a velocity formulation for solving quasi-static problems. The contact stresses are kept as variables and new variables are introduced in order to discriminate left and right sliding (see Klarbring (1999)) :

- $\lambda_{1}$ and $\lambda_{2}$ (respectively the positive and negative parts of the tangential displacements of the contact nodes),

- $\boldsymbol{\Phi}_{1}=-\mathbf{r}_{T}+\mu \mathbf{r}_{N}$ and $\boldsymbol{\Phi}_{2}=\mathbf{r}_{T}+\mu \mathbf{r}_{N}$.

The three inequations $\boldsymbol{\Phi}_{1} \geq 0, \boldsymbol{\Phi}_{2} \geq 0$ and $-\boldsymbol{r}_{N} \geq 0$ define the Coulomb cone.

A condensation is performed in order to eliminate the non contact variables and to reduce the size of the finite element problem. It consists in a partial inversion of the linear parts. Details can be found in Raous (1999). Then the complementarity problem is written as follows :

The complementary problem $\left(\boldsymbol{P}_{\text {Compl }}\right):$ Find $\boldsymbol{r} \in \mathbb{R}^{3 N_{c}}, \boldsymbol{u} \in \mathbb{R}^{3 N_{c}}$ such that

$$
\left.\begin{array}{l}
\widehat{M} \boldsymbol{u}=\widehat{\boldsymbol{F}}+\boldsymbol{r}, \\
\boldsymbol{r}_{i} \leq 0, \mathbf{u}_{i} \leq 0 \quad i=1 \ldots 3 N_{c} \\
\boldsymbol{r}_{i} \mathbf{u}_{i}=0 \quad i=1 \ldots 3 N_{c} .
\end{array}\right\}
$$

- $\widehat{M}$ and $\widehat{\boldsymbol{F}}$ are respectively a non symmetric matrix and a loading vector deduced from the finite element problem by condensation (taking also into account the change of variables associated to the choice of new variables),

- $\boldsymbol{r}$ and $\boldsymbol{u}$ are the contact forces $\left(\boldsymbol{r}_{N}, \boldsymbol{\Phi}_{1}, \boldsymbol{\Phi}_{2}\right)$ and the contact displacements $\left(\boldsymbol{u}_{N}, \lambda_{1}, \lambda_{2}\right)$

- $N_{c}$ is the number of contact particles.

This 2D formulation can be extended to 3D problems by performing a polyhedralization of the Coulomb cone (see Klarbring (1999)).

\section{c - Lagrange multipliers}

Another alternative is based, as with the previous formulation, on keeping the contact forces $r$ 
in the form of variables (Lagrange multipliers). This time, the unilateral conditions are kept in the form (3), but the friction conditions are written under the Kuhn-Tucker conditions (which is analogous to plasticity). This is written as problem $\left(\boldsymbol{P}_{\mathbf{L a g r}}\right)$, where $\widehat{K}$ is the condensed regular finite element matrix (see Chabrand-Dubois-Raous (1998)).

The Lagrangian problem $\left(\boldsymbol{P}_{\text {Lagr }}\right)$ : Find $r \in \mathbb{R}^{2 N_{c}}, \Delta \xi \in \mathbb{R}^{N_{c}}$ such that

$$
\left.\begin{array}{l}
\widehat{K} \mathbf{u}=\widehat{\boldsymbol{F}}+\boldsymbol{r} \\
\boldsymbol{u}_{N j} \leq 0, \quad \mathbf{r}_{N j} \leq 0, \quad \mathbf{u}_{N j} \mathbf{r}_{N j}=0, \quad j=1 \ldots N_{c}, \\
f_{s} \leq 0, \boldsymbol{\Delta} \boldsymbol{u}_{T}=-\boldsymbol{\Delta} \xi \partial f_{s} / \partial \mathbf{r}_{T}, \boldsymbol{\Delta} \xi \geq 0, \boldsymbol{\Delta} \xi f_{s}=0 \\
f_{s}=\left\|\mathbf{r}_{T}\right\|-\mu\left|r_{N}\right|
\end{array}\right\}
$$

\subsection{Solvers}

\section{a - The minimization problem under constraints}

Details about the following solvers can be found in Raous (1999).

To solve problem $\left(\boldsymbol{P}_{\text {opt } 1}\right)$ (or possibly $\left(\boldsymbol{P}_{\text {opt } 2}\right)$ ), the following algorithms have been developed. The minimization problem under constraints $(\forall v \in \mathcal{K})$ of a non differentiable functional can be solved using :

\section{- Successive over-relaxation method (SSOR) with projection}

An optimum relaxation parameter has to be determined using a trial procedure. This method is very robust, but it turns out to be costly when one attempts to extend it to problems involving nonlinear materials.

\section{- Gauss-Seidel method accelerated with an Aitken procedure}

No numerical parameters are required here. The method is a very robust and efficient one, specially with the recent computer processors very efficient on basic computations.

\section{- Conjugate gradient method with projection and regularization}

A special procedure is used to preserve the conjugation relationship between the descent directions (see Raous-Barbarin (1992)). To compute the gradients, a regularization of the friction law is needed ; this introduces a numerical parameter. Specific pre-conditioning procedures have been developed.

\section{b - The complementarity problem}

The complementarity problem $\left(\boldsymbol{P}_{\boldsymbol{C o m p l}}\right)$ can be solved using a mathematical programming method : the Lemke's method. This is a direct method based on pivoting techniques which is similar to Simplex methods. It is a very powerful method. No numerical parameter is required (see Klarbring (1999), Raous (1999)).

\section{c - Lagrangian formulation}

The problem $\left(\boldsymbol{P}_{\boldsymbol{L a g r}}\right)$ can be solved using the augmented Lagrangian method with radial return. The augmented Lagrangian procedure introduces a penalty by defining :

$$
\begin{aligned}
\boldsymbol{r}_{N} & =\left(\mathbb{\lambda}_{N}-\epsilon_{N} \boldsymbol{u}_{N}\right), \\
\boldsymbol{\Delta} \boldsymbol{r}_{T} & =\boldsymbol{\Delta} \mathbb{\lambda}_{T}-\epsilon_{T}\left(\mathbf{u}_{T}+\boldsymbol{\Delta} \xi \partial f_{s} / \partial \mathbf{r}_{T}\right) .
\end{aligned}
$$


A Newton Raphson algorithm is associated with a radial return procedure. This method is similar to procedures used in plasticity problems (even with finite deformations). It is a very powerful method. The accuracy of this method (as well as the computational cost) depends on the choice of $\epsilon_{T}, \epsilon_{N}$, and on the number of augmentations performed.

\subsection{Application to a block sliding on a plane}

A Polyurethane block is pressed (prescribed normal displacement) on an Araldite plane on which a tangential displacement is prescribed. The vertical displacement is applied in order to prescribe a constant normal force. The geometry is $80 \mathrm{~mm} \times 40 \mathrm{~mm}$. The elastic constants are $E=5 \mathrm{MPa}$ and $\nu=0.48$. The velocity of the plate is $v=0.103 \mathrm{~mm} / \mathrm{s}$ (see Zeghloul-Villechaise (1996)).

It can be noted from Fig. 6 and Fig. 7 that the evolution of the contact condition varies significantly depending on the values of $\mu$.

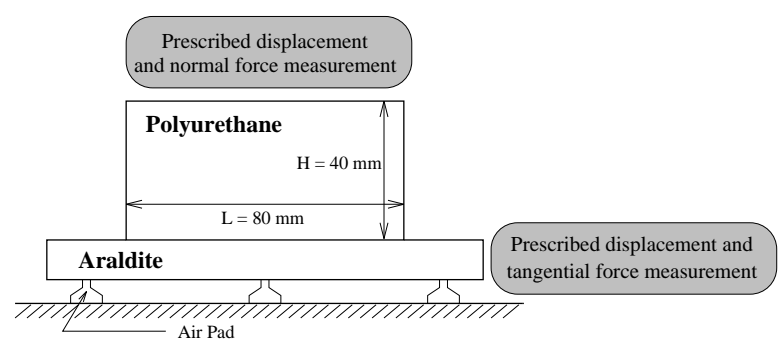

Figure 3. The model.

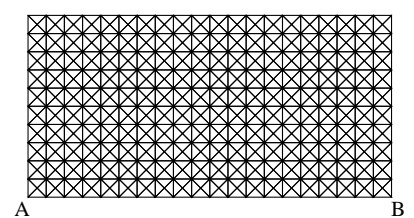

Figure 4. Mesh with 21 contact nodes.

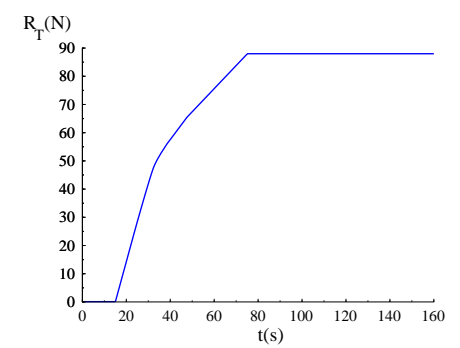

Figure 5. Evolution of the total friction force versus time (the tangential displacement speed is $0.103 \mathrm{~mm} / \mathrm{s}, \mu=1.1$ ). 

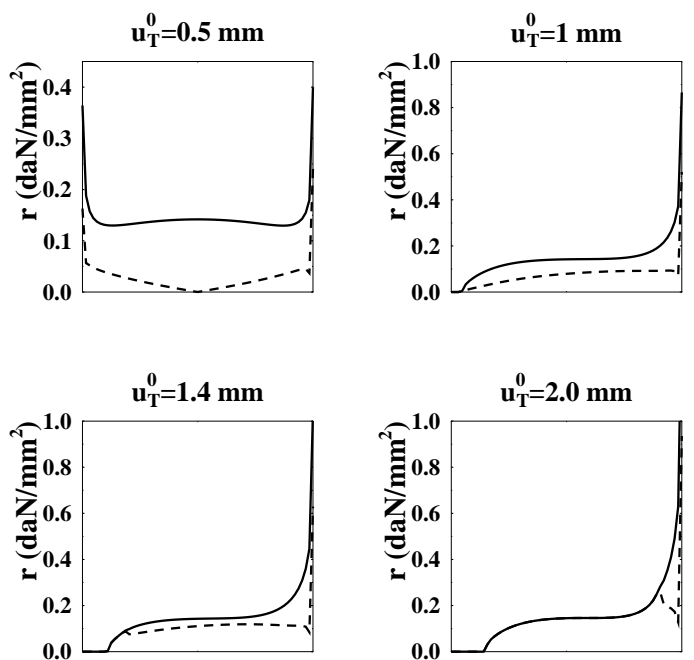

Figure 6. Evolution of the contact stresses $r_{N}(-)$ and $r_{T} / \mu(--)$ along the contact zone with $\mu=0.5$ (sticking, sliding and separated zones).
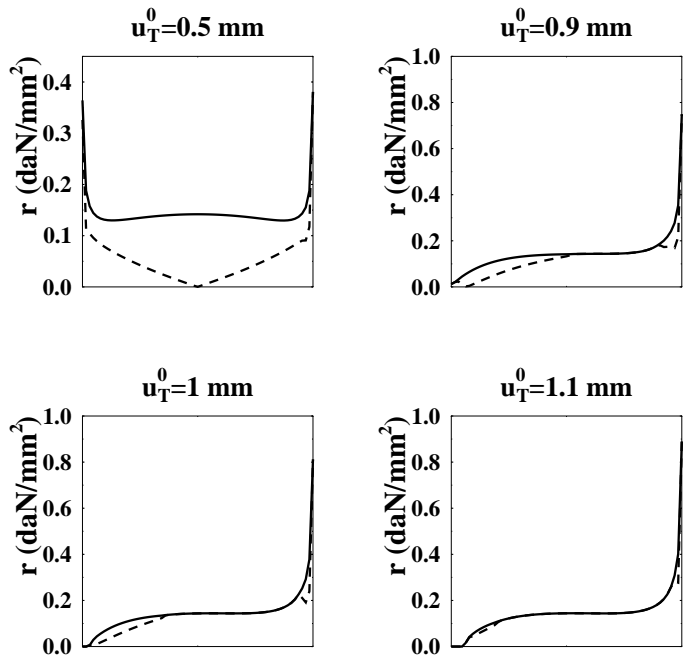

Figure 7. Evolution of the contact stresses $r_{N}(-)$ and $r_{T} / \mu(--)$ along the contact zone with $\mu=1$ (sticking, sliding and separated zones). 


\section{Dynamics for frictional contact problems involving small deformations}

In this section, dynamic problems involving unilateral contact and Coulomb friction will be briefly discussed. Focusing on the discretized problem, a formulation and an algorithm coupled with a mathematical programming method presented in Vola-Pratt-Jean-Raous (1998) are described. This approach is based on previous studies by J.J. Moreau and M. Jean (see JeanMoreau (1987), Jean (1999), Moreau (1988a), Moreau (1988b), Moreau (1994)).

\subsection{Viscoelastic contact problem with Coulomb friction in dynamics}

The dynamic Signorini problem with Coulomb friction is written as follows, where $\overline{\bar{C}}$ is the viscosity tensor and $\overline{\bar{K}}$ the elasticity tensor. The general formulation is given here but in the application the viscosity will not be considered.

Problem $\left(\boldsymbol{P}_{\boldsymbol{d y n}}\right)$ : Let $\Phi_{1}$ and $\Phi_{2}$ be given, find $u, \sigma$ and $r$ such that:

$$
\begin{aligned}
& \rho \ddot{u}(t)=\operatorname{div} \sigma(u(t))+\Phi_{1}(t), \quad \text { in } \Omega \\
& \sigma(u(t))=\overline{\bar{K}} \cdot \epsilon(u(t))+\overline{\bar{C}} \cdot \dot{\varepsilon}(u(t)), \quad \text { in } \Omega \\
& \varepsilon(u(t))=\frac{1}{2}\left(\nabla_{x}(u(t))+\nabla_{x}^{T}(u(t))\right), \quad \text { in } \Omega \\
& \dot{u}(t)=\dot{u}_{D}, \quad \text { on } \Gamma_{D} \\
& \sigma(u(t)) \cdot n=\Phi_{2}, \quad \text { on } \Gamma_{F} \\
& \sigma(u(t)) \cdot n=r(t), \quad \text { on } \Gamma_{C} \\
& u_{N}(t) \leq 0, r_{N}(t) \leq 0 \text { and } r_{N}(t) u_{N}(t)=0, \quad \text { on } \Gamma_{C} \\
& \left\|r_{T}(t)\right\| \leq \mu\left|r_{N}(t)\right| \text { and } \\
& \left\{\text { if }\left\|r_{T}(t)\right\|<\mu\left|r_{N}(t)\right| \text { then } \dot{u}_{T}(t)=0\right. \text {, } \\
& \text { if }\left\|r_{T}(t)\right\|=\mu\left|r_{N}(t)\right| \text { then } \exists \lambda>0 \text { such that } \dot{u}_{T}(t)=-\lambda r_{T}(t) \text {. }
\end{aligned}
$$

\subsection{The discrete problem (finite element discretization)}

In order to take impacts into account correctly, the formulation is given in terms of differential measures.

$\operatorname{Problem}\left(\boldsymbol{P}_{\boldsymbol{h}}\right)$ : Find $\boldsymbol{u}$ such that $\forall t \in[0, T], \boldsymbol{u}(t) \in \mathcal{V}_{h}, \boldsymbol{u}(0)=\boldsymbol{u}_{0}, \dot{\boldsymbol{u}}(0)=\boldsymbol{v}_{0}$ and :

$$
M . d \dot{\mathbf{u}}+K . \boldsymbol{u}+C \cdot \dot{\mathbf{u}}=\boldsymbol{\Phi}+\mathbf{r} d \nu,
$$

and for the contact nodes, $j=1 \ldots N_{c}$ ( $N_{c}$ is the number of contact nodes) :

$$
\begin{aligned}
& u_{N j}(t) \leq 0, r_{N j}(t) \leq 0 \text { and } r_{N j}(t) u_{N j}(t)=0 \\
& \left\|\mathbf{r}_{T j}(t)\right\| \leq \mu\left|r_{N j}(t)\right| \text { and }\left\{\begin{array}{l}
\text { if }\left\|\mathbf{r}_{T j}(t)\right\|<\mu\left|r_{N j}(t)\right| \text { then } \dot{\boldsymbol{u}}_{T j}(t)=0, \\
\text { if }\left\|\mathbf{r}_{T j}(t)\right\|=\mu\left|r_{N j}(t)\right| \text { then } \exists \lambda>0, \text { such that } \dot{\boldsymbol{u}}_{T j}(t)=-\lambda \boldsymbol{r}_{T j}(t),
\end{array}\right.
\end{aligned}
$$


where :

- $d \dot{\boldsymbol{u}}$ is a differential measure standing for the discretized acceleration

$$
\int_{] a, b]} d \dot{\boldsymbol{u}}=\dot{\boldsymbol{u}}^{+}(b)-\dot{\boldsymbol{u}}^{+}(a),
$$

with right continuity : we shall set $\dot{\boldsymbol{u}}=\dot{\boldsymbol{u}}^{+}$in the following,

$-d \nu$ is a non-negative real measure with respect to which $d \dot{\boldsymbol{u}}$ possesses a density function.

\subsection{Time discretization and algorithms}

The Newmark method and its variants are generally used to solve classical smooth problems (usual differential equations). In this section, for solving the non smooth problem $\left(\boldsymbol{P}_{\boldsymbol{h}}\right)$, a time discretization of (25) is introduced and $\theta$-methods will be used.

The system of differential measures (25) can be written in the following equivalent form : $\forall t \in[0, T]$

$$
\begin{gathered}
M(\dot{\boldsymbol{u}}(t)-\dot{\boldsymbol{u}}(0))=\int_{0}^{t}(\boldsymbol{\Phi}-K . \boldsymbol{u}-C . \dot{\boldsymbol{u}}) d s+\int_{[0, t]} \boldsymbol{r} d \nu, \\
\mathbf{u}(t)=\mathbf{u}(0)+\int_{0}^{t} \dot{\mathbf{u}} d s,
\end{gathered}
$$

where $d s$ is a Lebesgue measure.

A time discretization of (27), with $i=0 \ldots N$ and $t_{i}=i . h$, yields :

$$
\begin{gathered}
M\left(\dot{\boldsymbol{u}}\left(t_{i+1}\right)-\dot{\boldsymbol{u}}\left(t_{i}\right)\right)=\int_{t_{i}}^{t_{i+1}}(\boldsymbol{\Phi}-K . \boldsymbol{u}-C \cdot \dot{\boldsymbol{u}}) d s+\int_{] t_{i}, t_{i+1}\right]} \mathbf{r} d \nu \\
\overline{\mathbf{r}}^{i+1}=\frac{1}{h} \int_{] t_{i}, t_{i+1}\right]} \mathbf{r} d \nu .
\end{gathered}
$$

To complete the time discretization, the two following Lebesgue integrals must be approximated :

$$
\begin{gathered}
\int_{t_{i}}^{t_{i+1}}(\boldsymbol{\Phi}-K . \mathbf{u}-C . \dot{\boldsymbol{u}}) d s \\
\int_{t_{i}}^{t_{i+1}} \dot{\boldsymbol{u}} d s .
\end{gathered}
$$

The choice of integration methods is influenced by the fact that the velocity is discontinuous. The three following methods have been implemented and tested :

$\theta$-Method : both integrals (31) and (32) are approximated by the classical $\theta$-method

$$
\int_{t_{i}}^{t_{i}+1} f d s \approx h\left(\theta f\left(t_{i+1}\right)+(1-\theta) f\left(t_{i}\right)\right),
$$


$\theta$-Euler-Method : the first integral is approximated by the $\theta$-method and the second one by the implicit Euler method,

modified $\theta$-Method : both integrals are approximated by the $\theta$-method, but in the contact relations, the displacement $u\left(t_{i+1}\right)$ is replaced by $\widehat{u}\left(t_{i+1}\right)=u\left(t_{i+1}\right)+h(1-\theta) \dot{u}\left(t_{i+1}\right)$.

These three methods have been compared to the Newmark method and to the exact solution in a simple test, and we will comment on their respective advantages.

\subsection{Formulation in terms of complementarity}

Complementarity formulations for the frictional contact problem and mathematical programming algorithms can be found, for quasistatic problems, in Klarbring (1999) and Raous (1999).

Let us focus here on the extension of the complementarity formulation to the dynamic problem in order to combine the Lemke method with the previous integration methods.

As described in the previous section, we introduce the new variables :

$-\lambda_{1}$ and $\lambda_{2}$ (respectively, the positive and negative part of the tangential velocity),

$-\phi_{1}=-r_{T}+\mu r_{N}$,

$-\phi_{2}=+r_{T}+\mu r_{N}$.

The problem can be then written :

Problem $\left(\boldsymbol{P}_{\text {DynComp }}\right)$ : Find $\dot{\boldsymbol{u}}^{i+1} \in \mathcal{V}_{h}$ such that :

$$
\widetilde{M} \dot{\boldsymbol{u}}^{i+1}=h \widetilde{\boldsymbol{\Phi}}^{i+1}+h \boldsymbol{r}^{i+1},
$$

and for the contact nodes, $j=1 \ldots N_{c}$ :

$$
\begin{gathered}
r_{N j}{ }^{i+1} \leq 0, \quad \dot{u}_{N j}^{i+1}-\widetilde{G}_{j}^{i} \leq 0, r_{N j}{ }^{i+1} \cdot\left(\dot{u}_{N j}^{i+1}-\widetilde{G}_{j}^{i}\right)=0, \\
\phi_{1 j}{ }^{i+1} \leq 0, \quad \lambda_{1 j}{ }^{i+1} \leq 0, \phi_{1 j}{ }^{i+1} \cdot \lambda_{1 j}{ }^{i+1}=0 \\
\phi_{2 j}{ }^{i+1} \leq 0, \quad \lambda_{2 j}{ }^{i+1} \leq 0, \phi_{2 j}{ }^{i+1} \cdot \lambda_{2 j}{ }^{i+1}=0 .
\end{gathered}
$$

The matrix $\widetilde{M}$ and the vectors $\widetilde{\boldsymbol{\Phi}}^{i+1}$ and $\widetilde{\boldsymbol{G}}^{i}$ depend on the integration method adopted. For example :

- for the $\theta$-method, $\widetilde{M}=M+h \theta C+h^{2} \theta^{2} K$,

- whereas for the $\theta$-Euler-method, $\widetilde{M}=M+h \theta C+h^{2} \theta K$.

\subsection{Numerical example}

The classical benchmark of the impact of two bars is briefly presented. Fig. 9 gives the time evolution of the normal velocity of a central node of the contact zone, computed with the various integration methods (and the Lemke method). It can be observed that the $\theta$-Euler-method and the modified- $\theta$-method give much better results than the classical Newmark method. Oscillations can be observed with the Newmark method (see Fig. 9 where the exact solution is plotted as a dotted line). 

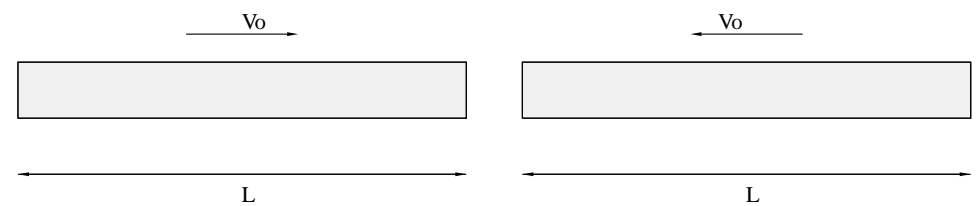

Figure 8. Impact between two bars.
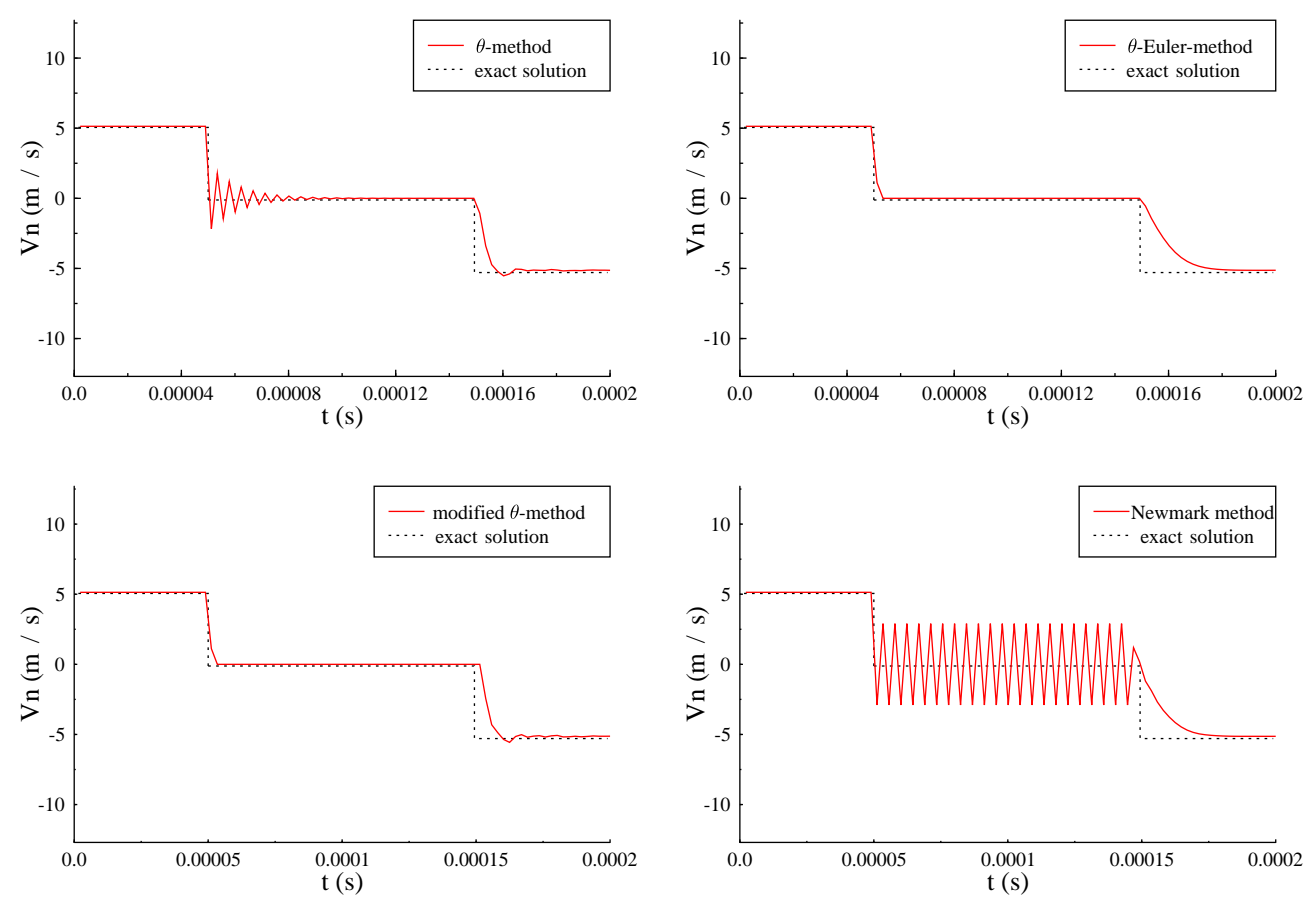

Figure 9. Normal velocity at the center of the contact zone. 


\section{Dynamics for frictional contact problems in hyper-elasticity}

The dynamic formulation presented in the previous section is now extended to finite deformations in the case of hyper-elastic problems (see Vola-Raous-Martins (1999)). Here, we outline the main points, as follows:

- the variational formulation in the current configuration,

- the discrete formulation in terms of differential measures,

- the nonlinear complementarity problem,

- the linearization : Newton-Raphson method, linear complementarity problem and Lemke solver.

\subsection{Formulation of the problem}

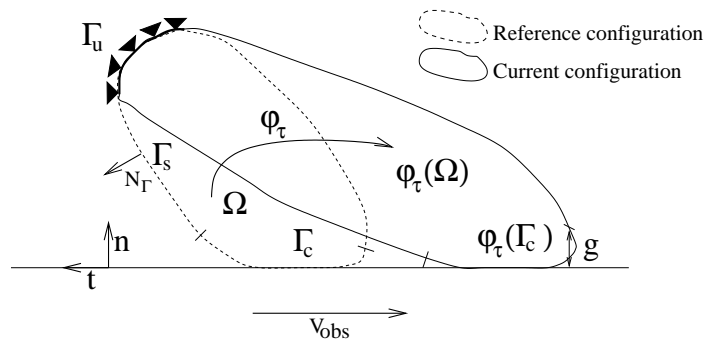

Figure 10. Contact of a deformable body with a flat obstacle.

\section{a - The problem}

\section{Equilibrium equations and frictional contact}

The equilibrium equations given below are based either on the reference configuration or on the current one.

$$
\begin{array}{llll}
\rho_{0} \ddot{\varphi}-\operatorname{Div}(F S)=f_{v} & \text { in } \Omega, & \rho \ddot{x}-\operatorname{div} \sigma=f_{v}^{\tau} \text { in } \varphi_{\tau}(\Omega), \\
(F S) N_{\Gamma}=f_{s} & \text { on } \Gamma_{s}, & \sigma n_{\varphi(\Gamma)}=f_{s}^{\tau} & \text { on } \varphi_{\tau}\left(\Gamma_{s}\right), \\
\varphi=\varphi_{\text {prescribed }} & \text { on } \Gamma_{u}, & x=x_{\text {prescribed }} & \text { on } \varphi_{\tau}\left(\Gamma_{u}\right), \\
(F S) N_{\Gamma}=R & \text { on } \Gamma_{c}, & \sigma n_{\varphi(\Gamma)}=r & \text { on } \varphi_{\tau}\left(\Gamma_{c}\right),
\end{array}
$$

where : $S$ is the second Piola-Kirchoff tensor, $\varphi$ the kinematic mapping, $F=\operatorname{grad} \varphi, J=\operatorname{det} F$, $C=F \cdot \bar{F}$ is the Cauchy-Green strain tensor and $\sigma$ the Cauchy stress tensor.

\section{Unilateral contact and Coulomb friction in the current configuration}

$$
\begin{gathered}
r_{n} \geq 0 \text { and } \forall r_{n}^{*} \geq 0, \quad\left(r_{n}^{*}-r_{n}\right) \omega_{n} \geq 0 \\
\left|r_{t}\right| \leq \mu r_{n} \text { and } \forall r_{t}^{*}\left|r_{t}^{*}\right| \leq \mu r_{n}, \quad\left(r_{t}^{*}-r_{t}\right) \dot{\omega}_{t} \geq 0
\end{gathered}
$$


where $\omega_{n}$ is the gap and $\dot{\omega}_{t}$ is the relative sliding velocity ( $n$ denotes now the outwards normal to the obstacle).

\section{Behaviour of rubber-like materials (hyper-elasticity)}

A Mooney-Rivlin behaviour is used :

$$
\widetilde{W}\left(\widetilde{I}_{1}, \widetilde{I}_{2}\right)=a_{1}\left(\widetilde{I}_{1}-3\right)+a_{2}\left(\widetilde{I}_{2}-3\right) .
$$

The constitutive law is written as follows in the reference configuration :

$$
S: \dot{E}=\frac{\partial W}{\partial E}: \dot{E} \quad \longrightarrow \quad S=2 \frac{\partial W}{\partial C} .
$$

The volumic deformation and the associated pressure are expressed with the two auxiliary fields $\theta$ and $p$ (see Simo-Taylor (1991)).

$$
\Pi_{\text {int }}=\int_{\Omega}\left(\widetilde{W}\left(\widetilde{I}_{1}, \widetilde{I}_{2}\right)+U(\theta)+p(J-\theta)\right) d V,
$$

denotes an augmented potential of the internal forces, where :

- $U(\theta)$ is a penalty function associated to the volumic deformation ( $\kappa$ is the bulk modulus)

$$
U(\theta)=\frac{1}{2} \kappa\left(\theta^{2}-1\right)-\ln (\theta),
$$

$-p$ is the pressure (Lagrange multiplier).

This is completed with the initial conditions and the mass conservation equation.

\section{b - Variational formulation}

The problem can be written in form of the coupling between a differential inclusion related to the motion and the friction, two variational equalities related to the auxiliary fields and a variational inequality related to the unilateral contact conditions.

In the reference configuration, the differential inclusion is written :

$$
-\Pi_{, \varphi}\left(\varphi_{\tau}, \theta, p, R_{n}\right)-\mathcal{F}_{\text {inertial }} \in \partial_{2} D\left(R_{n}, \dot{\varphi}_{\tau}-\Phi\right),
$$

where :

- the potential $\Pi$ is composed of three parts (internal stresses, external loading, normal contact force),

$$
\Pi\left(\varphi_{\tau}, \theta, p, R_{n}\right)=\Pi_{i n t}\left(\varphi_{\tau}, \theta, p\right)-\Pi_{e x t}\left(\varphi_{\tau}\right)-\int_{\Gamma_{c}} R_{n} W_{n}\left(\varphi_{\tau}\right) d \Gamma,
$$

with $\left(\varphi_{\tau}, \theta, p, R_{n}\right) \in \mathcal{W} \times \mathcal{V}^{2} \times \mathcal{C}_{n}$, where :

- $\mathcal{W}$ is the set of admissible configurations

$$
\mathcal{W}=\left\{\varphi_{\tau}: \Omega \longrightarrow \mathbb{R}^{2} \text { such that } \varphi_{\tau}(X)=\varphi_{\text {prescribed }} \quad \forall X \in \Gamma_{u}\right\} .
$$


- $\mathcal{V}$ is the set of admissible hydrostatic pressures and volume changes

$$
\mathcal{V}=\left\{\theta^{*}: \Omega \longrightarrow \mathbb{R}\right\}
$$

- $\mathcal{C}_{n}$ is the set of admissible normal contact stresses

$$
C_{n}=\left\{R_{n}^{*}: \Gamma_{c} \longrightarrow \mathbb{R} \text { such that } R_{n}^{*} \geq 0\right\} .
$$

- $D$ is the pseudo-potential associated with the friction dissipation (in the case of a plane obstacle),

$$
D\left(R_{n}, \dot{\varphi_{\tau}}-\Phi\right)=\int_{\Gamma_{c}} \mu R_{n}\left|\left(\dot{\varphi_{\tau}}-\Phi\right) \cdot t\right| d \Gamma,
$$

- and $\mathcal{F}_{\text {inertial }}$ are the inertial forces.

This is equivalent to the following variational form of the elasto-dynamic problem with frictional contact written now in the current configuration at the time $\tau$.

$\operatorname{Problem}\left(\boldsymbol{P}_{\boldsymbol{D} y \boldsymbol{n}}\right):$ Find $\left(\varphi_{\tau}, \dot{\varphi}_{\tau}, \ddot{\varphi_{\tau}}, \theta, p, r_{n}, r_{t}\right) \in \mathcal{W}^{3} \times \mathcal{V}^{2} \times \mathcal{C}_{n}^{\tau} \times \mathcal{C}_{t}^{\tau}\left(r_{n}\right)$ such that we have :

\section{Equation of motion}

$$
\begin{aligned}
\forall \eta^{\tau} \in T^{\tau} \mathcal{W}, & \int_{\varphi_{\tau}(\Omega)} \rho \ddot{\varphi}_{\tau} \eta^{\tau} d v+\int_{\varphi_{\tau}(\Omega)} \bar{\sigma}: \nabla_{x}^{S} \eta^{\tau} d v-\int_{\varphi_{\tau}(\Omega)} f_{V}^{\tau} \eta^{\tau} d v \\
& -\int_{\varphi_{\tau}\left(\Gamma_{S}\right)} f_{S}^{\tau} \eta^{\tau} d \Gamma-\int_{\varphi_{\tau}\left(\Gamma_{c}\right)}\left(r_{n} n+r_{t} t\right) \cdot \eta^{\tau} d \Gamma=0,
\end{aligned}
$$

Incompressibility (with the two auxiliary fields $(\theta, p)$ )

$$
\begin{gathered}
\forall \theta^{*} \in \mathcal{V}, \quad \int_{\varphi_{\tau}(\Omega)} \frac{1}{J}\left(U^{\prime}(\theta)-p\right) \theta^{*} d v=0, \\
\forall p^{*} \in \mathcal{V}, \quad \int_{\varphi_{\tau}(\Omega)}(J-\theta) \frac{p^{*}}{J} d v=0,
\end{gathered}
$$

Unilateral contact

$$
\forall r_{n}^{*} \in \mathcal{C}_{n}^{\tau}, \quad \int_{\varphi_{\tau}\left(\Gamma_{c}\right)}\left(r_{n}^{*}-r_{n}\right) \omega_{n} d \Gamma \geq 0
$$

Frictional contact (maximum dissipation principle)

$$
\forall r_{t}^{*} \in \mathcal{C}_{t}^{\tau}\left(r_{n}\right), \quad \int_{\varphi_{\tau}\left(\Gamma_{c}\right)}\left(r_{t}^{*}-r_{t}\right) \dot{\omega}_{t} d \Gamma \geq 0
$$

where $T^{\tau} \mathcal{W}$ is the set of admissible variations

$$
T^{\tau} \mathcal{W}=\left\{\eta^{\tau}: \varphi_{\tau}(\Omega) \longrightarrow \mathbb{R}^{2} \text { such that } \eta^{\tau}\left(\varphi_{\tau}(X)\right)=0 \forall X \in \Gamma_{u}\right\},
$$

and $C_{n}^{\tau}$ and $C_{t}^{\tau}\left(r_{n}\right)$ are the sets of admissible normal and tangential contact reactions

$$
\begin{aligned}
C_{n}^{\tau} & =\left\{r_{n}^{*}: \varphi_{\tau}\left(\Gamma_{c}\right) \longrightarrow \mathbb{R} \text { such that } r_{n}^{*} \geq 0\right\} \\
C_{t}^{\tau}\left(r_{n}\right) & =\left\{r_{t}^{*}: \varphi_{\tau}\left(\Gamma_{c}\right) \longrightarrow \mathbb{R} \text { such that }\left|r_{t}^{*}\right| \leq \mu r_{n}\right\}
\end{aligned}
$$

The present formulation with seven unknown fields is reduced to a three fields formulation in the case of the discrete problem. 


\subsection{The discrete problem}

\section{a - Space discretization}

Auxiliary fields $p$ and $\theta$ are eliminated at the finite element level. We use $Q_{4} P_{0}$ elements with four nodes for the displacements and one node for $p$ and $\theta$. We have :

$$
\theta_{e}^{h}=\operatorname{Vol}\left(\varphi_{\tau}\left(\Omega_{e}^{h}\right)\right) / \operatorname{Vol}\left(\Omega_{e}^{h}\right), \quad p_{e}^{h}=U^{\prime}\left(\theta_{e}^{h}\right) .
$$

After finite element discretization, the problem can be written as follows :

$\operatorname{Problem}\left(P_{D y n}^{h}\right):$

$$
\begin{gathered}
M \ddot{\boldsymbol{u}}+\boldsymbol{F}_{\text {int }}(\mathbf{u})-\boldsymbol{F}_{\text {ext }}(\mathbf{u})=H^{T} \boldsymbol{r}, \\
\forall \mathbf{r}_{n}^{*} \in \mathcal{C}_{n}^{h} \quad\left(\mathbf{r}_{n}^{*}-\boldsymbol{r}_{n}\right)^{T}\left(H_{n} \mathbf{u}+\boldsymbol{G}\right) \geq 0, \\
\forall \mathbf{r}_{t}^{*} \in \mathcal{C}_{t}^{h}\left(\boldsymbol{r}_{n}\right) \quad\left(\boldsymbol{r}_{t}^{*}-\boldsymbol{r}_{t}\right)^{T}\left(H_{t} \dot{u}-\boldsymbol{v}_{o b s}\right) \geq 0,
\end{gathered}
$$

where $H$ is the matrix which associates the local displacement vector (normal-tangential components $(n, t))$ with the global displacement vector :

$$
\left[\begin{array}{l}
r_{1} \\
r_{2}
\end{array}\right]_{i}=\underbrace{\left[\begin{array}{ll}
n_{1} t_{1} \\
n_{2} t_{2}
\end{array}\right]}_{H_{i}^{T}}\left[\begin{array}{l}
r_{n} \\
r_{t}
\end{array}\right]_{i},
$$

and where

$$
\begin{aligned}
\mathcal{C}_{n}^{h} & =\left\{\boldsymbol{r}_{n} \in \mathbb{R}^{N_{c}} \text { such that } r_{n_{i}} \geq 0 \forall i=1, . ., N_{c}\right\}, \\
\mathcal{C}_{t}^{h}\left(\mathbf{r}_{n}\right) & =\left\{\boldsymbol{r}_{t} \in \mathbb{R}^{N_{c}} \text { such that }\left|r_{t_{i}}\right| \leq \mu r_{n_{i}} \forall i=1, . ., N_{c}\right\} .
\end{aligned}
$$

\section{b - Time discretization}

As in the previous section, a formulation in terms of differential measures is used in order to take into account the velocity discontinuities induced by the impacts.

Equation of motion (50) is then written as follows (Pandit-Deo (1982)) :

$$
\forall \tau \in[0, T], \quad M d \dot{u}+\boldsymbol{F}_{\text {int }}(\mathbf{u}) d \tau-\boldsymbol{F}_{\text {ext }}(\mathbf{u}) d \tau-H^{T} \overline{\mathbf{r}} d \nu=0
$$

This nonlinear problem is a generalization of the dynamic problem set in linear elasticity in the previous section.

The previous differential equation (65) is approximated by using three different $\theta$-methods (Vola-Pratt-Jean-Raous (1998)) and the gap $\omega$ and the impulse term will be approximated by an implicit Euler method in order to prevent numerical oscillations after impact.

- Method $1: \theta$-method for the equation of motion and for the displacement.

- Method 2: Implicit Euler method for the equation of motion and the displacement.

- Method $3: \theta$-method for the equation of motion and implicit Euler method for the displacement 
In the following, method 1 will be used (with a single $\theta$ ).

Now, at each time step, we have to solve the non-linear complementarity problem written with the contact velocities and the contact forces.

$\operatorname{Problem}\left(\boldsymbol{P}_{\boldsymbol{m}}^{\boldsymbol{h}}\right):$ Find $\dot{\boldsymbol{u}}^{m+1} \in \mathbb{R}^{n_{d l}}, \overline{\mathbf{r}}_{n}^{m+1} \in \mathcal{C}_{n}^{h}$ and $\overline{\boldsymbol{r}}_{t}^{m+1} \in \mathcal{C}_{t}^{h}\left(\overline{\mathbf{r}}_{n}^{m+1}\right)$ such that :

$$
\begin{gathered}
M \dot{\boldsymbol{u}}^{m+1}+\Delta \tau \theta \boldsymbol{F}_{i n t}\left(l\left(\dot{\boldsymbol{u}}^{m+1}\right)\right)=\Delta \tau \theta \boldsymbol{F}_{\text {ext }}^{m+1}+\Delta \tau \boldsymbol{F}_{r e s}^{m}+\Delta \tau \overline{\mathbf{r}}^{m+1}, \\
\forall \overline{\mathbf{r}}_{n}^{*} \in \mathcal{C}_{n}^{h} \quad\left(\overline{\mathbf{r}}_{n}^{*}-\overline{\mathbf{r}}_{n}^{m+1}\right)^{T}\left(H_{n} \dot{\boldsymbol{u}}^{m+1}+\widetilde{\boldsymbol{G}}^{m}\right) \geq 0, \\
\forall \overline{\boldsymbol{r}}_{t}^{*} \in \mathcal{C}_{t}^{h}\left(\overline{\mathbf{r}}_{n}^{m+1}\right) \quad\left(\overline{\mathbf{r}}_{t}^{*}-\overline{\mathbf{r}}_{t}^{m+1}\right)^{T}\left(H_{t} \dot{\boldsymbol{u}}^{m+1}-\mathbf{v}_{o b s}\right) \geq 0,
\end{gathered}
$$

with :

$$
\begin{gathered}
l\left(\dot{\boldsymbol{u}}^{m+1}\right)=1 /(\theta \Delta \tau)\left(\boldsymbol{u}^{m+1}-\boldsymbol{u}^{m}\right)-(1-\theta) / \theta \dot{u}^{m} \\
\boldsymbol{F}_{r e s}^{m}=1 / \Delta \tau M \dot{\boldsymbol{u}}^{m}+(1-\theta)\left[\boldsymbol{F}_{\text {ext }}^{m}-\boldsymbol{F}_{\text {int }}\left(l\left(\dot{\boldsymbol{u}}^{m}\right)\right)\right] .
\end{gathered}
$$

\section{c - Solvers}

The nonlinear problem $\left(\boldsymbol{P}_{\boldsymbol{m}}^{\boldsymbol{h}}\right.$ ) is linearized (see Josephy (1979)) and a Newton-Raphson method is used. At each Newton iterate, the following linearized complementarity problem will be solved with the Lemke solver.

Problem $\left(\boldsymbol{P}_{\text {linearized }}\right)$ : Let $\left(\dot{\boldsymbol{u}}, \overline{\mathbf{r}}_{n}, \overline{\boldsymbol{r}}_{t}\right)^{m}$ the solution previously computed up to and including the time $\tau^{m}$, find $\left(\dot{\boldsymbol{u}}, \overline{\mathbf{r}}_{n}, \overline{\mathbf{r}}_{t}\right)^{m+1}$, the limit of the sequence $\left(\dot{\boldsymbol{u}}_{k+1}, \overline{\mathbf{r}}_{n_{k+1}}, \overline{\mathbf{r}}_{t_{k+1}}\right) \in \mathbb{R}^{n_{d l}} \times \mathcal{C}_{n}^{h} \times$ $\mathcal{C}_{t}^{h}\left(\overline{\boldsymbol{r}}_{n_{k}}\right)$ where :

$$
\begin{gathered}
H \hat{K}_{T_{k}} H^{T} H d \dot{\boldsymbol{u}}_{k+1}=H \boldsymbol{F}_{R e s}^{m}-\frac{1}{\Delta \tau} H M \dot{\boldsymbol{u}}_{k}+\theta H\left[\boldsymbol{F}_{\text {ext }}-\boldsymbol{F}_{\text {int }}\left(l\left(\dot{\boldsymbol{u}}_{k}\right)\right)\right]+\overline{\mathbf{r}}_{k+1} \\
\forall \overline{\mathbf{r}}_{n}^{*} \in \mathcal{C}_{n}^{h} \quad\left(\overline{\boldsymbol{r}}_{n}^{*}-\overline{\mathbf{r}}_{n_{k+1}}\right)^{T}\left(H_{n} \dot{\boldsymbol{u}}_{k}+\widetilde{\boldsymbol{G}}^{m}+H_{n} d \dot{\boldsymbol{u}}_{k+1}\right) \geq 0 \\
\forall \overline{\boldsymbol{r}}_{t}^{*} \in \mathcal{C}_{t}^{h}\left(\overline{\mathbf{r}}_{n_{k+1}}\right) \quad\left(\overline{\mathbf{r}}_{t}^{*}-\overline{\boldsymbol{r}}_{t_{k+1}}\right)^{T}\left(H_{t} \dot{\boldsymbol{u}}_{k}+H_{t} d \dot{\boldsymbol{u}}_{k+1}-\boldsymbol{v}_{o b s}\right) \geq 0
\end{gathered}
$$

where $\widehat{K}_{T_{k}}$ is the tangent stiffness matrix and $k$ denotes the Newton iterate.

This form of the dynamic problem leads to a sequence of problems very similar to those obtained for a static problem but including some extra terms related to the solution at the previous time step. This is very important for the solvers.

These problems are solved using the Lemke method presented in the first section.

\subsection{The quasistatic problem : a particular case of the dynamic problem}

For the quasistatic problem, the acceleration terms are neglected. It is then easy to adapt the formulations and the solvers presented in the previous section for solving the quasistatic problem.

$\operatorname{Problem}\left(\boldsymbol{P}_{\mathbf{Q u a s i s t a t i c}}\right):$ Find $\left(\varphi_{\tau}, \dot{\varphi_{\tau}}, \theta, p, r_{n}, r_{t}\right) \in \mathcal{W}^{2} \times \mathcal{V}^{2} \times \mathcal{C}_{n}^{\tau} \times \mathcal{C}_{t}^{\tau}\left(r_{n}\right)$ such that :

$$
\begin{aligned}
\forall \eta^{\tau} \in T^{\tau} \mathcal{W}, & \int_{\varphi_{\tau}(\Omega)} \bar{\sigma}: \nabla_{x}^{S} \eta^{\tau} d v-\int_{\varphi_{\tau}(\Omega)} f_{V}^{\tau} \eta^{\tau} d v-\int_{\varphi_{\tau}\left(\Gamma_{S}\right)} f_{S}^{\tau} \eta^{\tau} d \Gamma \\
& -\int_{\varphi_{\tau}\left(\Gamma_{c}\right)}\left(r_{n} n+r_{t} t\right) \cdot \eta^{\tau} d \Gamma=0,
\end{aligned}
$$




$$
\begin{gathered}
\forall \psi \in \mathcal{V}, \quad \int_{\varphi_{\tau}(\Omega)} \frac{1}{J}\left(U^{\prime}(\theta)-p\right) \psi d v=0, \\
\forall q \in \mathcal{V}, \quad \int_{\varphi_{\tau}(\Omega)}(J-\theta) \frac{q}{J} d v=0, \\
\forall r_{n}^{*} \in \mathcal{C}_{n}^{\tau}, \quad \int_{\varphi_{\tau}\left(\Gamma_{c}\right)}\left(r_{n}^{*}-r_{n}\right) \omega_{n} d \Gamma \geq 0, \\
\forall r_{t}^{*} \in \mathcal{C}_{t}^{\tau}\left(r_{n}\right), \quad \int_{\varphi_{\tau}\left(\Gamma_{c}\right)}\left(r_{t}^{*}-r_{t}\right) \dot{\omega}_{t} d \Gamma \geq 0 .
\end{gathered}
$$

After time and space discretization and linearization, the discrete quasistatic problem can be written in the following linear form (79).

Problem $\left(\boldsymbol{P}_{\text {Quasistatic-Linearized }}\right):$ Let $\left(\boldsymbol{u}, \overline{\boldsymbol{r}}_{n}, \overline{\boldsymbol{r}}_{t}\right)^{m}$ the quasistatic solution is known up to time $\tau^{m}$, find $\left(\boldsymbol{u}, \overline{\mathbf{r}}_{n}, \overline{\mathbf{r}}_{t}\right)^{m+1}$ limit of the sequence $\left(\boldsymbol{u}_{k+1}, \overline{\mathbf{r}}_{n_{k+1}}, \overline{\mathbf{r}}_{t_{k+1}}\right) \in \mathbb{R}^{n_{d l}} \times \mathcal{C}_{n}^{h} \times \mathcal{C}_{t}^{h}\left(\overline{\mathbf{r}}_{n_{k}}\right)$ where:

$$
\begin{gathered}
\left.H \widehat{K}_{T_{k}} H^{T} H d \boldsymbol{u}_{k+1}=H \boldsymbol{F}_{e x t}-H \boldsymbol{F}_{i n t}\left(\mathbf{u}_{k}\right)\right)+\overline{\mathbf{r}}_{k+1} \\
\overline{\boldsymbol{r}}_{n_{k+1}} \in \mathcal{C}_{n}^{h} \text { and } \forall \overline{\mathbf{r}}_{n}^{*} \in \mathcal{C}_{n}^{h}\left(\overline{\mathbf{r}}_{n}^{*}-\overline{\mathbf{r}}_{n_{k+1}}\right)^{T}\left(H_{n} \mathbf{u}_{k}+H_{n} d \mathbf{u}_{k+1}+\boldsymbol{G}^{m}\right) \geq 0, \\
\overline{\boldsymbol{r}}_{t_{k+1}} \in \mathcal{C}_{t}^{h}\left(\overline{\boldsymbol{r}}_{n_{k+1}}\right) \text { and } \forall \overline{\boldsymbol{r}}_{t}^{*} \in \mathcal{C}_{t}^{h}\left(\overline{\mathbf{r}}_{n_{k+1}}\right)\left(\overline{\mathbf{r}}_{t}^{*}-\overline{\boldsymbol{r}}_{t_{k+1}}\right)^{T}\left(H_{t} \Delta \mathbf{u}_{k}+H_{t} d \mathbf{u}_{k+1}\right) \geq 0,
\end{gathered}
$$

with $d \boldsymbol{u}_{k+1}=\Delta \boldsymbol{u}_{k+1}-\Delta \boldsymbol{u}_{k}$, where $\Delta \boldsymbol{u}_{k}$ is the displacement increment between the previous step and the iterate $k$ of the Newton-Raphson algorithm.

\subsection{Steady sliding solution for a set of values of the friction coefficient}

The steady sliding problem is more simple because the particles candidate for contact are either separated, or sliding with relative velocity opposite to that of the obstacle $\left(v_{o b s}\right)$. The Coulomb law is then simply written as follows :

$$
\mathbf{r}_{t}=\mu \operatorname{sgn}\left(v_{o b s}\right) \mathbf{r}_{n} .
$$

The discrete steady sliding problem is therefore written as follows :

Problem $\left(P_{\text {Steady-Sliding }}\right):$ Find $\boldsymbol{u} \in \mathbb{R}^{n_{d l}}$ and $\boldsymbol{r}_{n} \in \mathcal{C}_{n}^{h}$ such that :

$$
\begin{aligned}
& \boldsymbol{F}_{\text {int }}(\boldsymbol{u})=H_{n}^{T} \boldsymbol{r}_{n}+\mu \operatorname{sgn}\left(v_{o b s}\right) H_{t}^{T} \boldsymbol{r}_{n}, \\
& \forall \mathbf{r}_{n}^{*} \in \mathcal{C}_{n}^{h} \quad\left(\mathbf{r}_{n}^{*}-\boldsymbol{r}_{n}\right)^{T}\left(\boldsymbol{G}+H_{n} \mathbf{u}\right) \geq 0,
\end{aligned}
$$

and a fixed point procedure can be used on $\boldsymbol{r}_{t}$.

When stability analysis of steady sliding solutions will be conducted, the influence of the friction coefficient will be of great relevance. The following algorithm is therefore used to compute directly the steady sliding solutions with a set of various friction coefficient values. Each solution is then used as an initial condition for solving the next problem. 


\section{Algorithm}

- Incrementation of the friction coefficient.

- The problem is solved by using a fixed point method on the friction force :

$$
\begin{gathered}
\left(\boldsymbol{u}^{0}, \boldsymbol{r}_{n}^{0}\right), . .,\left(\mathbf{u}^{l-1}, \boldsymbol{r}_{n}^{l-1}\right) \text { being known, find } \boldsymbol{u}^{l} \in \mathbb{R}^{n_{d l}} \text { and } \boldsymbol{r}_{n}^{l} \in \mathcal{C}_{n}^{h} \text { such that : } \\
\boldsymbol{F}_{\text {int }}\left(\mathbf{u}^{l}\right)=H_{n}^{T} \boldsymbol{r}_{n}^{l}+H_{t}^{T} \boldsymbol{r}_{t}^{l-1} \\
\forall \boldsymbol{r}_{n}^{*} \in \mathcal{C}_{n}^{h} \quad\left(\boldsymbol{r}_{n}^{*}-\boldsymbol{r}_{n}^{l}\right)^{T}\left(\boldsymbol{G}^{m}+H \boldsymbol{u}\right) \geq 0
\end{gathered}
$$

with $\boldsymbol{r}_{t}^{l-1}=\mu \operatorname{sgn}\left(v_{o b s}\right) H_{t}^{T} \mathbf{r}_{n}^{l-1}$.

- Initial condition : the previous steady sliding solution obtained with the previous value of the friction coefficient.

The validity of the algorithm has been confirmed by computing the complete quasistatic solution until the the steady sliding solution was obtained (see Section 3.6).

\subsection{Validation on test examples}

\section{a - Quasistatic computation of the compression of a cylinder}

This benchmark was described by Simo-Taylor (1991), Sussman-Bathe (1987) and Liu-HofstetterMang (1994). The following computations were conducted by Didier Vola (Vola (1998)). Results obtained for frictionless and frictional $(\mu=0.2)$ cases are presented in Fig. 11, 12 and 13. The results show an excellent agreement with those obtained by Sussman-Bathe (displacement/hydrostatic pressure formulation) and Simo-Taylor.

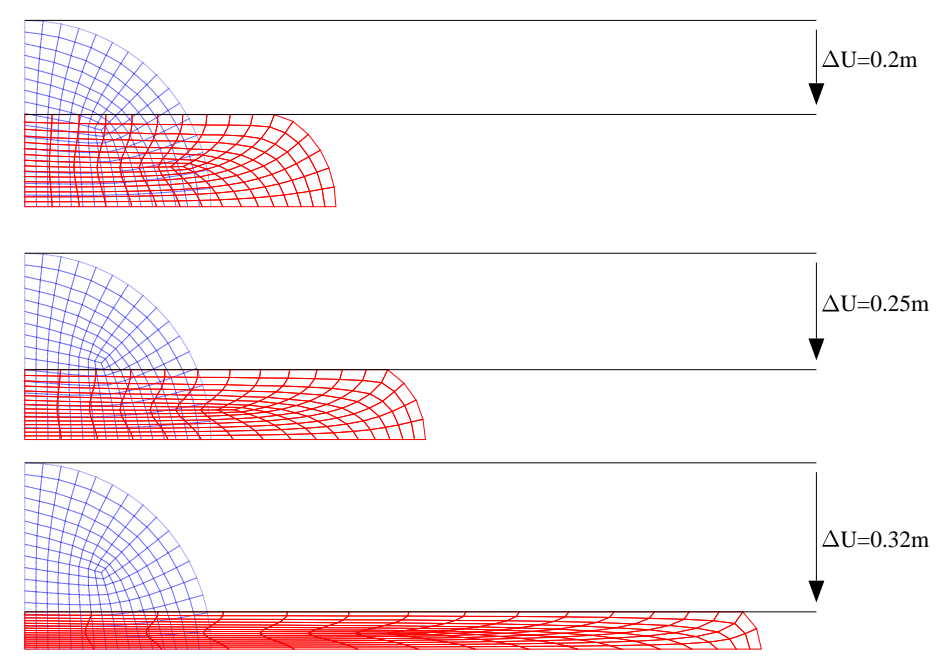

Figure 11. Deformations in the frictionless case. 


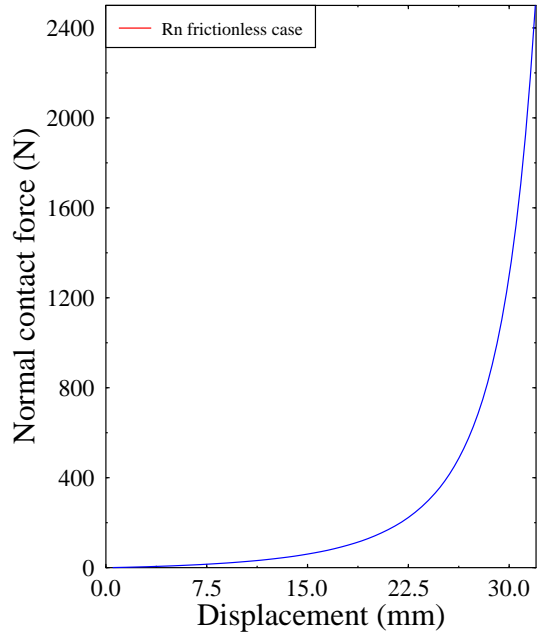

Figure 12. Total contact forces at $\mu=0$.

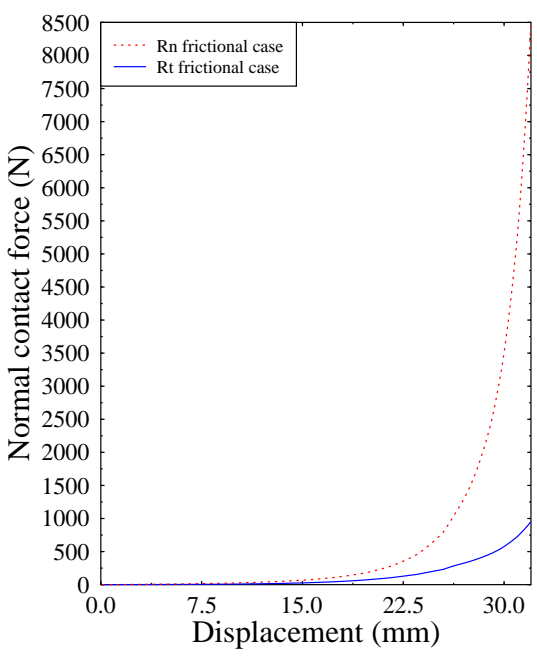

Figure 13. Total contact forces at $\mu=0.2$.

\section{b - Dynamic impact of a cylinder into an angle}

This benchmark was proposed by Wriggers-Vu Van-Stein (1990). The model is given in Fig. 15. Results obtained with various friction coefficient values are presented in Fig. 16. The velocitiy oscillations presented in Fig. 14 characterize the vibrations of the cylinder when it jumps back out from the angle (except for $\mu=0.5$, where it remains stuck).

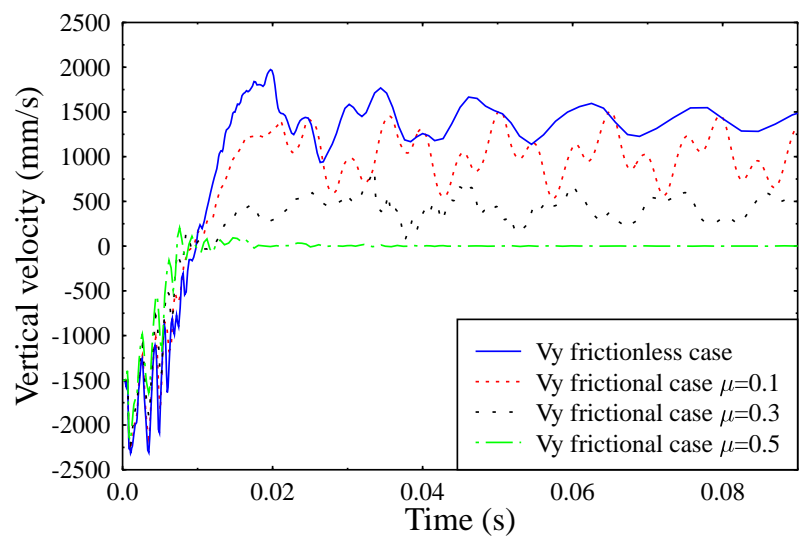

Figure 14. Evolution of the tangential velocity of node 1 with $\mu=0, \mu=0.1, \mu=0.3$ and $\mu=0.5$. 


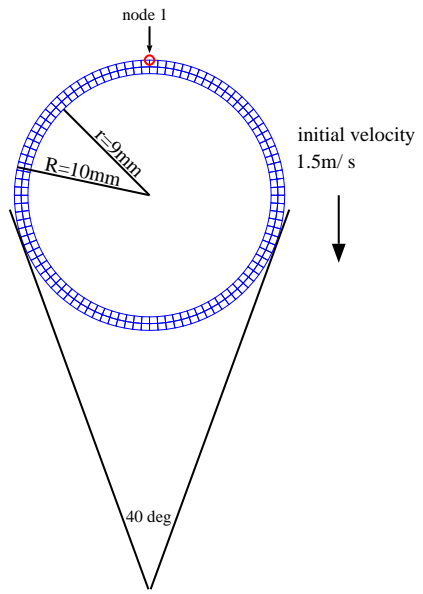

Figure 15. The model.
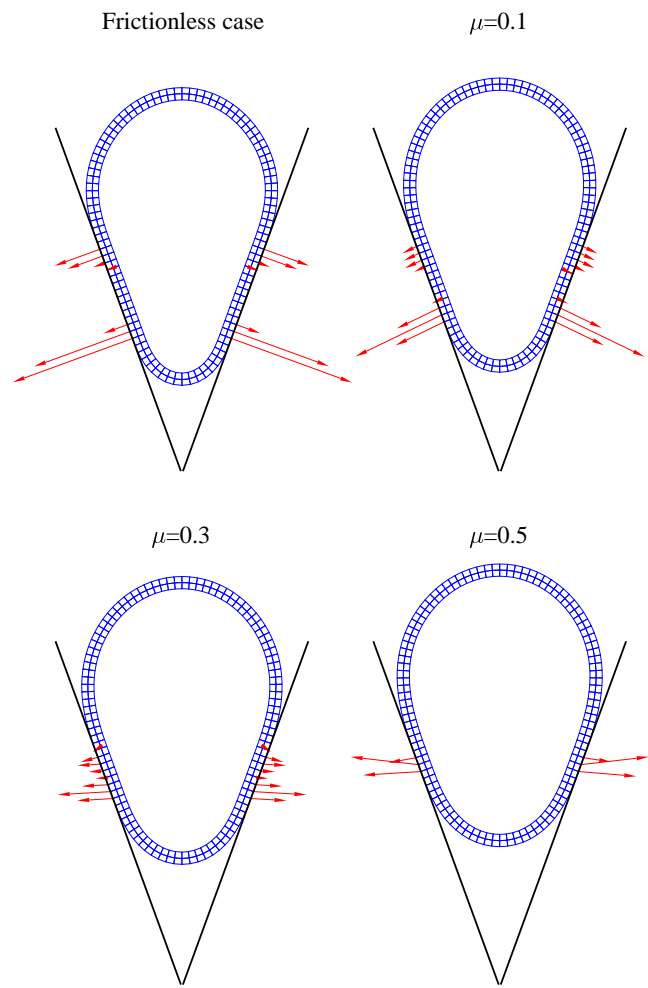

Figure 16. Deformations and contact forces with $\mu=0, \mu=0.1, \mu=0.3$ and $\mu=0.5$ at $\mathrm{t}=0.085 \mathrm{~s}$. 


\subsection{Efficiency of the algorithm when computing the steady sliding solution}

In this example, which is a simplified waist seal geometry, the steady sliding solution was computed either using the direct algorithm presented in Section 3.4 or as the asymptotic solution of the quasistatic problem, when the algorithm presented in Section 3.3 was used. Comparisons on the accuracy given on Fig. 19 and Fig. 20 and on the CPU times given on Table 2 (where $\epsilon$ is the value for the convergence test on the fixed point iterations on the friction forces), show that the direct method for computing the steady sliding solution is the most efficient. It is worth mentioning that the steady sliding solutions were computed taking various friction coefficient values, whereas the quasistatic solution was computed with only one value of the friction coefficient.

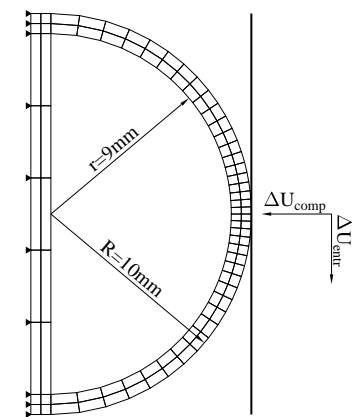

Figure 17. The model.

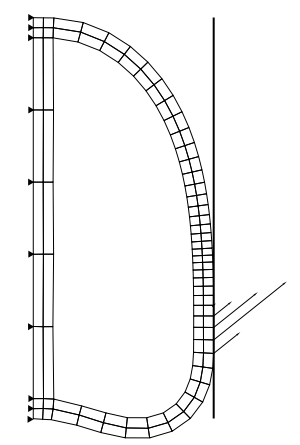

Figure 18. Steady sliding solution for $\mu=0.8$.

\begin{tabular}{|l|c|}
\hline First Mooney-Rivlin coefficient & $0.293 \mathrm{MPa}$ \\
\hline Second Mooney-Rivlin coefficient & $0.177 \mathrm{MPa}$ \\
\hline Compressibility modulus & $1200 \mathrm{MPa}$ \\
\hline Volume variation tolerance & $0.01 \%$ \\
\hline Plane strain hypothesis
\end{tabular}

Table 1. Material characteristics.

\begin{tabular}{|l|l|l|l|}
\hline Method & $\begin{array}{l}\text { Number of displacement } \\
\text { increments of the window }\end{array}$ & $\begin{array}{l}\text { Number of steady sliding } \\
\text { solutions (various } \mu \text { ) }\end{array}$ & CPU time \\
\hline \hline Quasistatic (reference) & 1920 of $0.110^{-2} \mathrm{~mm}$ & 1 & 756.7 sec. \\
\hline Quasistatic & 10 of $0.3 \mathrm{~mm}$ & 1 & 31.6 sec. \\
\hline \hline Direct $\epsilon=10^{-3}$ & & 10 with $\Delta \mu=0.08$ & 37.2 sec. \\
\hline Direct $\epsilon=10^{-5}$ & & 10 with $\Delta \mu=0.08$ & 63 sec. \\
\hline Direct $\epsilon=10^{-5}$ & & 320 with $\Delta \mu=0.2510^{-2}$ & 574,5 sec. \\
\hline
\end{tabular}

Table 2. Comparison between the computational times required by the two methods 


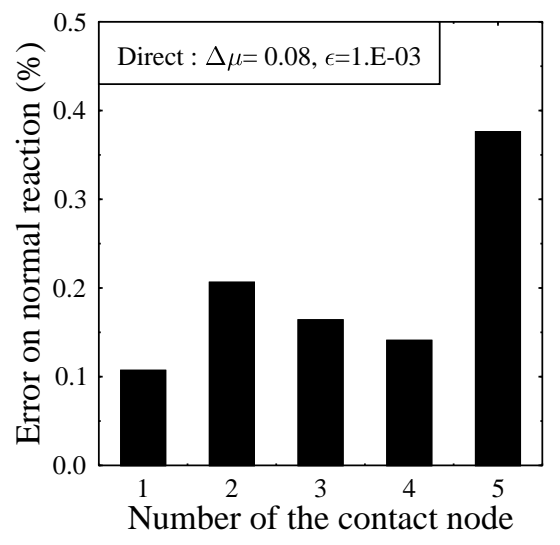

Figure 19. Error between the steady sliding solution and a reference asymptotic quasistatic one ( 1920 increments) : normal reactions.

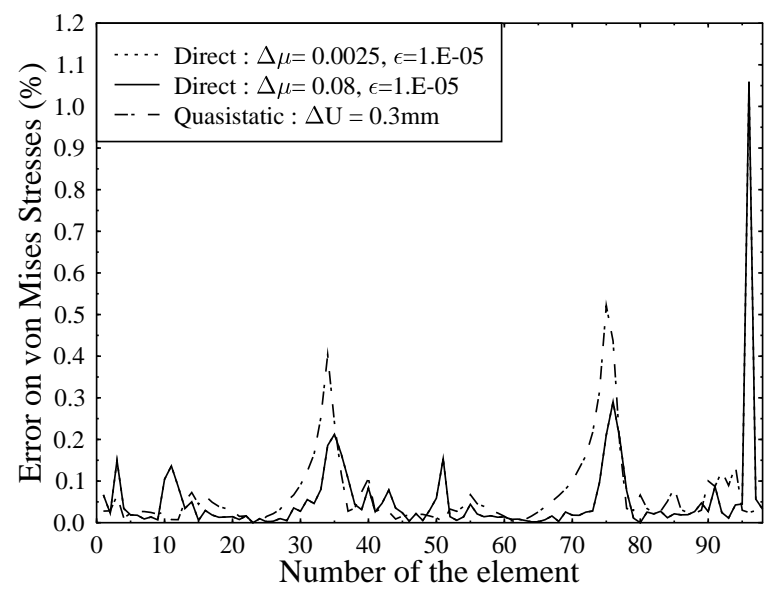

Figure 20. Error on the mean value of the Von Mises stresses by elements, influence of the number of increments

\subsection{The steady sliding solution for the waist seal}

In this section, the steady sliding solution of two kinds of waist seals sliding on a glass is presented. The stability of these solutions will be analyzed in the last section. The Mooney-Rivlin constitutive parameters are $a_{1}=0.293 \mathrm{MPa}, a_{2}=0.177 \mathrm{MPa}$ and $\kappa=1200 \mathrm{MPa}$. For small deformation, in linear elasticity, it corresponds to $E=4.58 \mathrm{MPa}$ and $\nu=0.4995$. The behaviour is quasi-incompressible (the volumic deformation will be under $0.01 \%$ with this value of $\kappa$ ). The 
volumic mass is $\rho=1200 \mathrm{Kg} / \mathrm{m}^{3}$ and the velocity of the glass is $V_{\text {glass }}=80 \mathrm{~mm} / \mathrm{s}$. The meshes of the two models of waist seals are given in Fig. 21 and Fig. 22. The steady sliding solutions for the glass moving up and the glass moving down are given in Fig. 23 for geometry 1, and in Fig. 24 for geometry 2 .

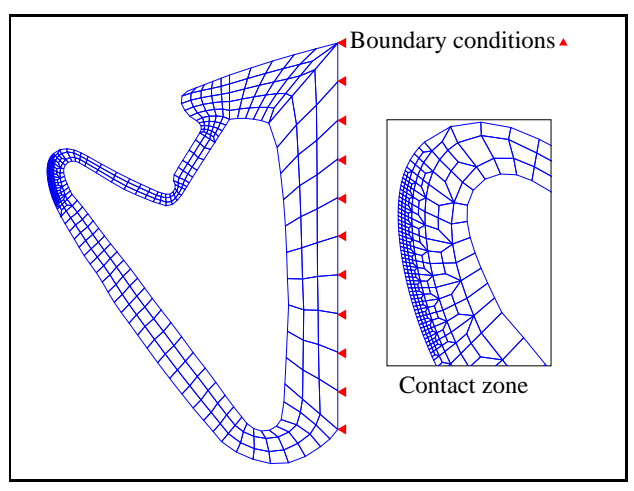

Figure 21. Mesh of the waist seal $n^{\circ} 1$ and zoom of the contact zone.

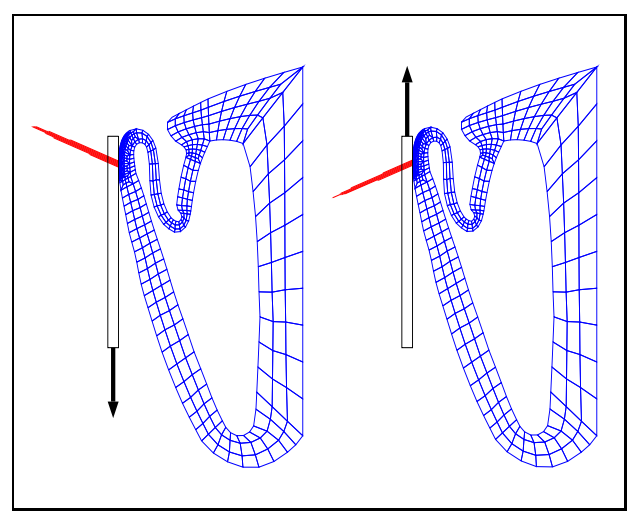

Figure 23. Steady sliding solution and contact forces, geometry 1 with $\mu=0.4$.

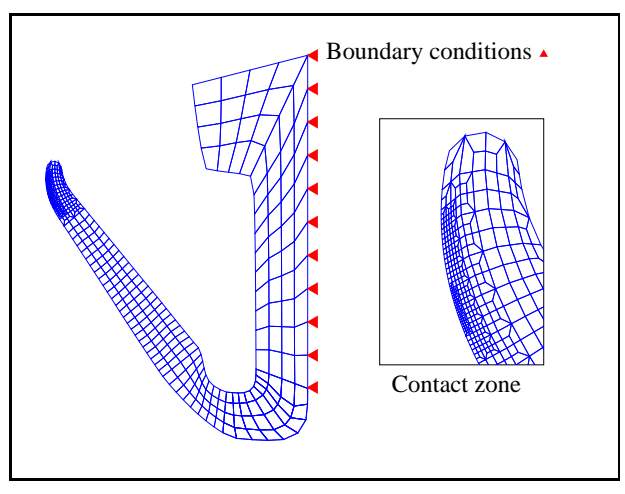

Figure 22. Mesh of the waist seal $\mathrm{n}^{\circ} 2$ and zoom of the contact zone.

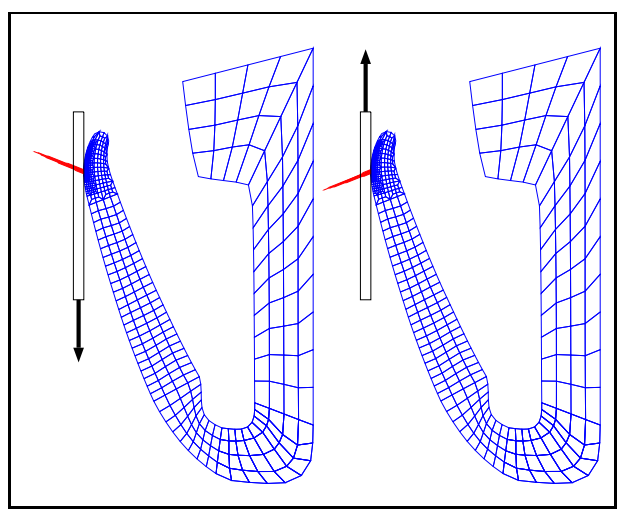

Figure 24. Steady sliding solution and contact forces, geometry 2 with $\mu=0.4$. 


\section{Numerical analysis of the stability for quasistatic solutions of frictional contact problems in linear elasticity}

\subsection{Condition for dynamic growth in the neighborhood of quasi-static paths}

\section{a - Introduction}

First, we recall the main results established in Martins et al (1999) and Barbarin (1997), for finite dimensional problems, on the stability of quasistatic solutions of linear elastic problems involving frictional contact. We will focus here on the computation of the various stability conditions and on their application to the example of a Polyurethane block sliding on a plane given in Section 1.

In general, a quasistatic evolution is not a solution of the dynamic problem, except if the velocity $\dot{\boldsymbol{u}}^{0}(t)$ is constant.

We now consider straight portions of quasistatic evolutions. Thus, we assume that the variation of the applied forces is linear (see (87)), and that the quasistatic evolution $\left(\boldsymbol{u}^{0}(t), \mathbf{r}^{0}(t)\right)$ has the linear variation given by (88) and (89) in some time interval $\left[\tau, \tau+\Delta^{0} \tau[\right.$ :

$$
\begin{gathered}
f(t)=f(\tau)+\dot{f}(\tau)(t-\tau), \text { for } t \geq \tau, \\
\boldsymbol{u}^{0}(t)=\boldsymbol{u}^{0}(\tau)+\dot{\boldsymbol{u}}^{0+}(\tau)(t-\tau), \\
\boldsymbol{r}^{0}(t)=\mathbf{r}^{0}(\tau)+\dot{\boldsymbol{r}}^{0+}(\tau)(t-\tau) .
\end{gathered}
$$

As commented in Martins et al (1999) (Remark 4.5) the following results apply then also to steady sliding solutions.

In some right neighborhood $\left[\tau, \tau+\Delta \tau\left[\right.\right.$ of $\tau$, with $0<\Delta \tau \leq \Delta^{0} \tau$, we look for dynamic solutions having the form

$$
\mathbf{u}(t)=\mathbf{u}^{0}(t)+\alpha(t) \boldsymbol{A}, \quad \boldsymbol{r}(t)=\mathbf{r}^{0}(t)+\beta(t) \boldsymbol{B},
$$

with

$$
\begin{gathered}
\boldsymbol{A} \in \mathcal{K}_{\ddot{\boldsymbol{u}}}\left(\mathbf{u}^{0}(\tau), \boldsymbol{r}^{0}(\tau), \dot{\boldsymbol{u}}^{0+}(\tau), \dot{\boldsymbol{r}}^{0+}(\tau)\right), \\
\boldsymbol{B} \in \mathcal{K}_{\ddot{\boldsymbol{r}}}\left(\boldsymbol{u}^{0}(\tau), \boldsymbol{r}^{0}(\tau), \dot{\boldsymbol{u}}^{0+}(\tau), \dot{\boldsymbol{r}}^{0+}(\tau), \boldsymbol{A}\right),
\end{gathered}
$$

where $\mathcal{K}_{\ddot{\mathbf{u}}}$ and $\mathcal{K}_{\ddot{\mathbf{r}}}$ are defined by (97) and (98), and :

- $\alpha$ is a twice continuously differentiable function such that $\alpha$ and $\dot{\alpha}$ are non-negative and non-decreasing in the interval defined above,

- $\beta$ is continuous, non-negative and non-decreasing in the same interval,

- the initial values $\alpha(\tau) \geq 0, \dot{\alpha}(\tau) \geq 0$ are arbitrarily small.

\section{b - Admissible directions}

The admissible directions, in which the perturbed solution can possibly evolve, are defined by the following sets (see Martins et al (1999) where details on the notations will be found): 
- for the displacements and the contact forces :

$$
\begin{aligned}
& \mathcal{V}_{\boldsymbol{u}} \stackrel{\text { def }}{=}\left\{\boldsymbol{u} \in \mathbb{R}^{N}: u_{i}=0, \text { for all } i \in \mathcal{S}_{D}\right\}, \\
& \mathcal{V}_{\mathbf{r}} \stackrel{\text { def }}{=}\left\{\boldsymbol{r} \in \mathbb{R}^{N}: r_{i}=0, \text { for all } i \in \mathcal{S}_{F}\right\}
\end{aligned}
$$

$$
\begin{aligned}
& \mathcal{K}_{\boldsymbol{u}}=\left\{\boldsymbol{u} \in \mathcal{V}_{\mathbf{u}} \text { such that } u_{n_{i}} \geq 0 \quad \forall i \in \mathcal{P}_{C}\right\} \\
& \mathcal{K}_{\boldsymbol{r}}(\mathbf{u})=\left\{\boldsymbol{r} \in \mathcal{V}_{\mathbf{r}} \text { such that } r_{n_{i}}=r_{t_{i}}=0 \quad \forall i \in \mathcal{P}_{f}(\mathbf{u})\right. \\
& \left.r_{n_{i}} \leq 0 \text { and }\left|r_{t_{i}}\right| \leq \mu r_{n_{i}} \quad \forall i \in \mathcal{P}_{c}(\mathbf{u})\right\}
\end{aligned}
$$

where :

- $\mathcal{S}_{D}$ and $\mathcal{S}_{F}$ are respectively the set of degrees of freedom with prescribed displacement and the one of free displacement,

- $\mathcal{P}_{C}=\mathcal{P}_{f}(\boldsymbol{u}) \cup \mathcal{P}_{c}(\boldsymbol{u})$ is the set of contact particles,

- $\mathcal{P}_{c}(\boldsymbol{u})=\left\{i \in \mathcal{P}_{C}: u_{n_{i}}=0\right\}$ is the set of particles currently in contact,

- $\mathcal{P}_{f}(\boldsymbol{u})=\left\{i \in \mathcal{P}_{C}: u_{n_{i}}>0\right\}$ is the set of particles currently separated,

- for the velocities and the contact force rates :

$$
\begin{aligned}
\mathcal{K}_{\dot{u}}(\boldsymbol{u}, \boldsymbol{r})=\{ & \left\{\boldsymbol{v} \in \mathcal{V}_{\boldsymbol{u}}\right. \text { such that } \\
& v_{n_{i}} \geq 0 \quad \forall i \in \mathcal{P}_{z}(\boldsymbol{u}, \boldsymbol{r}) \\
& v_{n_{i}}=v_{t_{i}}=0 \quad \forall i \in \mathcal{P}_{d}(\boldsymbol{u}, \boldsymbol{r}) \\
& \left.v_{n_{i}}=0,-\operatorname{sgn}\left(r_{t_{i}}\right) v_{t_{i}} \geq 0 \quad \forall i \in \mathcal{P}_{s}(\boldsymbol{u}, \boldsymbol{r})\right\},
\end{aligned}
$$

$$
\begin{aligned}
& \mathcal{K}_{\dot{\mathbf{r}}}(\boldsymbol{u}, \boldsymbol{r}, \mathbf{v})=\left\{\boldsymbol{w} \in \mathcal{V}_{\mathbf{r}}\right. \text { such that } \\
& w_{n_{i}} \geq 0 \quad w_{n_{i}} v_{n_{i}}=0 \quad w_{t_{i}} \in \mu w_{n_{i}} \sigma\left(v_{t_{i}}\right) \quad \forall i \in \mathcal{P}_{z}(\boldsymbol{u}, \boldsymbol{r}) \\
& w_{n_{i}}=w_{t_{i}}=0 \quad \forall i \in \mathcal{P}_{f}(\boldsymbol{u}) \\
& -w_{t_{i}} \operatorname{sgn}\left(r_{t_{i}}\right)+\mu w_{n_{i}} \geq 0 \text {, } \\
& \left.\left(-w_{t_{i}} \operatorname{sgn}\left(r_{t_{i}}\right)+\mu w_{n_{i}}\right) \operatorname{sgn}\left(r_{t_{i}}\right) v_{t_{i}}=0 \quad \forall i \in \mathcal{P}_{s}(\boldsymbol{u}, \boldsymbol{r})\right\} .
\end{aligned}
$$

where :

- $\mathcal{P}_{z}(\boldsymbol{u}, \boldsymbol{r})=\left\{i \in \mathcal{P}_{c}(\boldsymbol{u}): r_{n_{i}}=r_{t_{i}}=0\right\}$ is the set of particles in contact with zero reaction,

- $\mathcal{P}_{d}(\boldsymbol{u}, \boldsymbol{r})=\left\{i \in \mathcal{P}_{c}(\boldsymbol{u}): r_{n_{i}}<0\right.$ and $\left.\left|r_{t_{i}}\right|<-\mu r_{n_{i}}\right\}$ is the set of particles in contact currently stuck,

- $\mathcal{P}_{s}(\boldsymbol{u}, \boldsymbol{r})=\left\{i \in \mathcal{P}_{c}(\boldsymbol{u}): r_{n_{i}}<0\right.$ and $\left.\left|r_{t_{i}}\right|=-\mu r_{n_{i}}\right\}$ is the set of particles in contact currently sliding,

- for the accelerations and the second-order contact force rates :

$$
\begin{aligned}
K_{\ddot{\mathbf{u}}}(\mathbf{u}, \boldsymbol{r}, \mathbf{v}, \mathbf{w})=\left\{\mathbf{a} \in \mathcal{V}_{\mathbf{u}}:\right. & a_{N p} \leq 0, \text { for } p \in \mathcal{P}_{z z} ; \\
& a_{N p}=0, \text { for } p \in \mathcal{P}_{d} \cup \mathcal{P}_{s} \cup \mathcal{P}_{z s} \cup \mathcal{P}_{z d} ; \\
& a_{T p} \sigma\left(r_{T p}\right) \leq, \text { for } p \in \mathcal{P}_{s s} \cap \mathcal{P}_{0} ; \\
& a_{T p} \sigma\left(w_{T p}\right) \leq 0, \text { for } p \in \mathcal{P}_{z s} \cap \mathcal{P}_{0} ; \\
& \left.a_{T p}=0, \text { for } p \in \mathcal{P}_{d} \cup \mathcal{P}_{s d} \cup \mathcal{P}_{z d}\right\}
\end{aligned}
$$




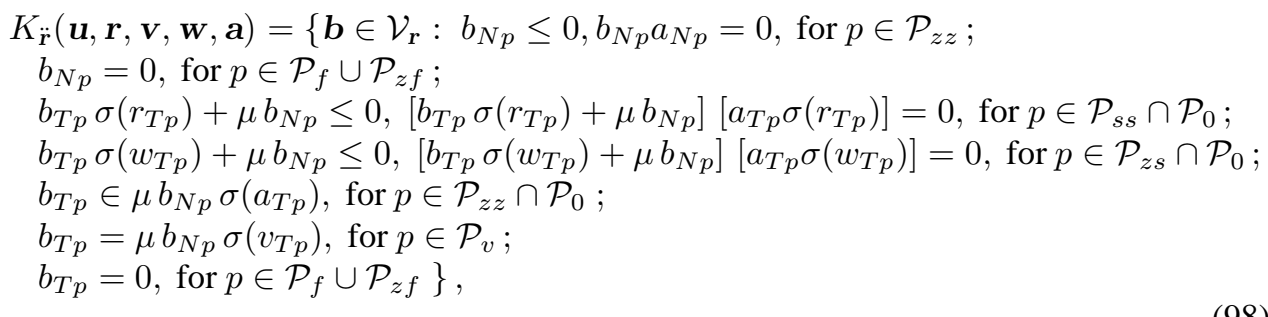

where

- $\mathcal{P}_{0}=\mathcal{P}_{0}\left(u^{0}(\tau), \dot{u}^{0+}(\tau)\right)$ and $\mathcal{P}_{v}=\mathcal{P}_{v}\left(u^{0}(\tau), \dot{u}^{0+}(\tau)\right)$ are the two complementary sets of particles in contact with zero normal velocity. $\mathcal{P}_{0}$ is the set of particles with tangential velocity equal to 0 and $\mathcal{P}_{v}$ the set of particle with non-vanishing tangential velocity,

- $\mathcal{P}_{z z}, \mathcal{P}_{z d}, \mathcal{P}_{z s}$, and $\mathcal{P}_{z f}$ are the set of particles in grazing contact (vanishing contact forces) that are respectively the one staying in the same situation, the one evolving to stuck situation, the one beginning to slide and the one going to be separated in the next future,

- $\mathcal{P}_{s s}$ and $\mathcal{P}_{s d}$ are the sets of sliding particles that are respectively remaining so and evolving to stuck situation.

Hereafter, the above sets of admissibility will be denoted by $\mathcal{K}_{\ddot{u}}(\cdots)$ and $\mathcal{K}_{\ddot{r}}(\cdots, \boldsymbol{A})$ for simplicity's sake.

\section{c - Conditions for growth of dynamic solutions or instability}

Let us summarize here the results established in Martins et al (1999) where the various proofs can be found.

For each $(\boldsymbol{A}, \mathbf{v}) \in \mathcal{K}_{\ddot{u}}(\cdots) \times \mathcal{K}_{\ddot{u}}(\cdots)$, we use the notations :

$$
\begin{gathered}
m^{* *}(\boldsymbol{A}, \mathbf{v}) \stackrel{\text { def }}{=} M \boldsymbol{A} . \mathbf{v}-\sum_{p \in \mathcal{P}_{v}} \mu \sigma\left(\dot{u}_{T p}^{0+}(\tau)\right)[M \boldsymbol{A}]_{N p} v_{T p}-\sum_{p \in \mathcal{P}_{0}} \mu[M \boldsymbol{A}]_{N p}\left|v_{T p}\right|, \\
a^{* *}(\boldsymbol{A}, \mathbf{v}) \stackrel{\text { def }}{=} K \boldsymbol{A} . \mathbf{v}-\sum_{p \in \mathcal{P}_{v}} \mu \sigma\left(\dot{u}_{T p}^{0+}(\tau)\right)[K \boldsymbol{A}]_{N p} v_{T p}-\sum_{p \in \mathcal{P}_{0}} \mu[K \boldsymbol{A}]_{N p}\left|v_{T p}\right| .
\end{gathered}
$$

where

$$
\sigma(x) \stackrel{\text { def }}{=} \begin{cases}x /|x|, & \text { if } x \neq 0 \\ {[-1,1],} & \text { if } x=0 .\end{cases}
$$

and where $M$ and $K$ denote respectively the mass and stiffness matrices.

The following result has been given in (Martins et al (1999)) :

\section{Proposition 1.}

If $\quad \exists \lambda \geq 0$ and $\boldsymbol{A} \in \mathcal{K}_{\ddot{u}}(\cdots), \boldsymbol{A} \neq 0$,

such that 


$$
\left(\lambda^{2} M+K\right) \boldsymbol{A} \in \mathcal{K}_{\ddot{\mathbf{r}}}(\cdots, \boldsymbol{A}),
$$

i.e., such that

$$
\lambda^{2}\left[m^{* *}(\boldsymbol{A}, \mathbf{v})-m^{* *}(\boldsymbol{A}, \mathbf{v})\right]+\left[a^{* *}(\boldsymbol{A}, \boldsymbol{A})-a^{* *}(\boldsymbol{A}, \boldsymbol{A})\right] \geq 0, \quad \forall \mathbf{v} \in \mathcal{K}_{\ddot{\boldsymbol{u}}}(\cdots),
$$

then there exists a dynamic solution of the form (90) in $[\tau, \tau+\Delta \tau[$, with

$$
\begin{gathered}
\boldsymbol{B}=\left(\lambda^{2} M+K\right) \boldsymbol{A}, \\
\beta(t)=\alpha(t)=\left\{\begin{array}{l}
\alpha(\tau) \cosh [\lambda(t-\tau)]+\left[\frac{\dot{\alpha}(\tau)}{\lambda}\right] \sinh [\lambda(t-\tau)], \text { if } \lambda>0, \\
\alpha(\tau)+\dot{\alpha}(\tau)(t-\tau), \text { if } \lambda=0,
\end{array}\right.
\end{gathered}
$$

with $\Delta \tau \leq \Delta^{0} \tau$, positive and sufficiently small, for all arbitrary, sufficiently small $\alpha(\tau) \geq 0$, $\dot{\alpha}(\tau) \geq 0$.

We now assume that the following restriction holds (details on the exact sets definitions can be found in Martins et al (1999))

$$
\mathcal{P}_{z z} \cup\left[\left(\mathcal{P}_{z s} \cup \mathcal{P}_{s s}\right) \cap \mathcal{P}_{0}\right]=\emptyset
$$

which means that :

- particles in grazing contact could not stay in the same situation $\left(\mathcal{P}_{z z}=\emptyset\right)$,

- particles in contact for which the current or the near future reactions do not vanish, and are on the boundary of the Coulomb friction cone, could not have a vanishing tangential velocity $\left(\mathcal{P}_{z s} \cup \mathcal{P}_{s s}\right) \cap \mathcal{P}_{0}=\emptyset$.

Consequently, no inequality restriction remains on the second-order displacement and reaction rates in both $\mathcal{K}_{\ddot{\boldsymbol{u}}}(\cdots)$ and $\mathcal{K}_{\ddot{\mathbf{r}}}(\cdots, \boldsymbol{A})$ that become subspaces of $\mathbb{R}^{N}$. In this situation, we now seek for growing dynamic solutions in the neighborhood of the quasistatic path in the form :

$$
\mathbf{u}(t)=\mathbf{u}^{0}(t)+\operatorname{Re}[\alpha(t) \boldsymbol{A}], \quad \boldsymbol{r}(t)=\boldsymbol{r}^{0}(t)+\operatorname{Re}[\beta(t) \boldsymbol{B}],
$$

with

$$
\boldsymbol{A} \in \mathcal{V}_{\ddot{u}}^{\mathbb{C}}, \quad \boldsymbol{B} \in \mathcal{V}_{\ddot{\mathrm{r}}}^{\mathbb{C}},
$$

where $\mathcal{V}_{\ddot{u}}^{\mathbb{C}}$ and $\mathcal{V}_{\ddot{r}}^{\mathbb{C}}$ are subspaces of admissible complex second order displacement and reaction rates.

For each $(\boldsymbol{A}, \mathbf{v}) \in \mathcal{V}_{\dot{\mathbf{u}}}^{\mathbb{C}} \times \mathcal{V}_{\dot{u}}^{\mathbb{C}}$, we define the following sesquilinear forms :

$$
\begin{gathered}
m^{\sharp}(\boldsymbol{A}, \mathbf{v}) \stackrel{\text { def }}{=} M \boldsymbol{A} \cdot \boldsymbol{v}-\sum_{p \in \mathcal{P}_{z s} \cup \mathcal{P}_{s s}} \mu \sigma\left(\dot{u}_{T p}^{0+}(\tau)\right)[M \boldsymbol{A}]_{N p} \bar{v}_{T p} \\
a^{\sharp}(\boldsymbol{A}, \mathbf{v}) \stackrel{\text { def }}{=} K \boldsymbol{A} \cdot \boldsymbol{v}-\sum_{p \in \mathcal{P}_{z s} \cup \mathcal{P}_{s s}} \mu \sigma\left(\dot{u}_{T p}^{0+}(\tau)\right)[K \boldsymbol{A}]_{N p} \bar{v}_{T p},
\end{gathered}
$$

where $\mathcal{P}_{z s} \cup \mathcal{P}_{s s}$ are particles in contact with a non-vanishing tangential velocity and a nonvanishing reaction on the friction cone, or a vanishing reaction and a non-vanishing reaction rate on the friction cone.

In these definitions, the term $A . v$ defines the inner product of complex vectors.

Then, we have the following result : 
Proposition 2. If (105) holds and if $\exists \lambda \in \mathbb{C}$ with $\operatorname{Re}(\lambda)>0$ such that one of the following equivalent conditions holds :

$$
\text { (i) } \exists \boldsymbol{A} \in \mathcal{V}_{\ddot{\mathbf{u}}}^{\mathbb{C}}, \boldsymbol{A} \neq 0 \text {, such that } \quad\left(\lambda^{2} M+K\right) \boldsymbol{A} \in \mathcal{V}_{\ddot{\mathbf{r}}}^{\mathbb{C}}
$$

(ii) $\exists \boldsymbol{A} \in \mathcal{V}_{\ddot{\mathbf{u}}}^{\mathbb{C}}, \boldsymbol{A} \neq 0$, such that $\quad \lambda^{2} m^{\sharp}(\boldsymbol{A}, \boldsymbol{A})+a^{\sharp}(\boldsymbol{A}, \boldsymbol{A})=0, \quad \forall \boldsymbol{A} \in \mathcal{V}_{\ddot{\mathbf{u}}}^{\mathbb{C}}$

then, there exists a dynamic solution of the form (106) in $[\tau, \tau+\Delta \tau[$, with

$$
\begin{gathered}
\boldsymbol{B}=\left(\lambda^{2} M+K\right) \boldsymbol{A}, \\
\beta(t)=\alpha(t)=\alpha(\tau) \cosh [\lambda(t-\tau)]+\left[\frac{\dot{\alpha}(\tau)}{\lambda}\right] \sinh [\lambda(t-\tau)],
\end{gathered}
$$

with $\Delta \tau \leq \Delta^{0} \tau$, positive and sufficiently small, for all arbitrary, sufficiently small $|\alpha(\tau)|>0$ and $|\dot{\alpha}(\tau)|>0$.

The statements of Propositions 1 and 2 are not interpreted as instability results because they only refer to a portion of a quasistatic evolution that is also a dynamic solution in some possibly finite time interval $\left[\tau, \tau+\Delta^{0} \tau\right.$. An extra hypothesis is then necessary to obtain the following stability result.

Proposition 3. If $\left(u^{0}(t), r^{0}(t)\right)$ given by (88-89) solves the quasistatic problem and consequently the dynamic problem for all $t \in[\tau,+\infty[$ and, if the assumptions of Proposition 1 and 2 hold, then the solution $\left(u^{0}(t), r^{0}(t)\right), t \geq \tau$ is dynamically unstable. If $\operatorname{Im}(\lambda)=0$, we have a divergence and if $\operatorname{Im}(\lambda) \neq 0$ we have flutter.

\section{d - Construction of the mass and stiffness matrices involved in the stability analysis}

We will now interpret the above necessary and sufficient conditions for divergence instabilities in terms of the properties of mass and stiffness matrices introduced before.

In practice because of the space and time discretization involved in the numerical resolution of the quasistatic problem and because of the finite digit accuracy, condition (105) holds and the following sets of particles are empty :

- the set $\mathcal{P}_{z}\left(u^{0}(\tau), \dot{u}^{0+}(\tau)\right)$ of particles in contact with a zero reaction (grazing contact),

- the set of particles in contact with non-zero reaction on the friction cone and zero tangential velocity.

In that case there is no distinction between $m^{* *}$ and $m^{\sharp}$ and between $a^{* *}$ and $a^{\sharp}$. Then the analysis needs the construction of matrices $M^{*}$ and $K^{*}$ associated to these bilinear forms and defined below.

We have now three remaining sets of contact particles :

- contact particles currently free (separated) $\mathcal{S}_{f}$,

- contact particles currently in contact with reactions strictly inside the friction cone (stuck) $\mathcal{P}_{d}$, 
- contact particles currently in contact with non-vanishing reactions on the friction cone and a non-vanishing tangential velocity (sliding) $\mathcal{P}_{s s}$.

In order to construct $M^{*}$ and $K^{*}$, we start by eliminating the non active degrees of freedom, which are :

- both degrees of freedom of the nodes with prescribed displacements (component $i \in S_{D}$ ),

- both degrees of freedom of the contact nodes that are stuck,

- the normal degree of freedom of the contact nodes that are sliding.

Let us denote by $S^{*}$ the set of degrees of freedom that may be right active and by $N^{*}$ the corresponding total number of degrees of freedom. The matrices $M^{*}$ and $K^{*}$ are then defined by :

For all $j \in S^{*}:$

- $M_{i, j}^{*}=M_{i, j}$ and $K_{i, j}^{*}=K_{i, j}$ for all degrees of freedom $i \in S^{*}$ that are not tangential degrees of freedom of particles $p$ in $\mathcal{P}_{s s}$,

- and for all particles $p$ in $\mathcal{P}_{s s}$ (set of the sliding particles) :

$$
M_{T p, j}^{*}=M_{T p, j}-\mu \sigma\left(\dot{u}_{T p}^{0+}(\tau)\right) M_{N p, j}, \quad K_{T p, j}^{*}=K_{T p, j}-\mu \sigma\left(\dot{u}_{T p}^{0+}(\tau)\right) K_{N p, j}(.1
$$

The symmetrized matrices $M_{S}^{*}$ and $K_{S}^{*}$ will be useful for the necessary condition. They are given by :

$$
M_{S}^{*}=\frac{1}{2}\left(M^{*}+M^{* T}\right), \quad K_{S}^{*}=\frac{1}{2}\left(K^{*}+K^{* T}\right)
$$

e - The various conditions for dynamic growth : the generalized eigenvalue problem The conditions given in Propositions 1, 2 and 3 will now be interpreted in terms of properties of matrices $M^{*}, K^{*}, M_{S}^{*}$ and $K_{S}^{*}$. Details about the establishment of the above propositions can be found in Martins et al (1999).

Proposition 4. Necessary condition for divergence

$$
\begin{array}{r}
\text { If } M_{S}^{*} \text { is positive semi-definite, and } \\
\text { if } K_{S}^{*} \text { is positive definite, }
\end{array}
$$

then growing perturbed dynamic solution of the form (90)) cannot occur.

\section{Proposition 5. Sufficient condition for divergence}

$$
\begin{array}{r}
\text { If } \exists \lambda \geq 0 \text { and } \boldsymbol{V}^{*} \in \mathcal{K}_{\ddot{u}}^{*}(\cdots), \boldsymbol{V}^{*} \neq 0, \\
\text { such that }\left(\lambda^{2} M^{*}+K^{*}\right) \boldsymbol{V}^{*}=0,
\end{array}
$$

then (101) holds with the same value of $\lambda$ and

$$
\boldsymbol{V}=\left\{\begin{array}{l}
\boldsymbol{V}_{D} \\
\boldsymbol{V}_{d} \\
\boldsymbol{V}_{s N} \\
\boldsymbol{V}^{*}
\end{array}\right\}=\left\{\begin{array}{c}
0 \\
0 \\
0 \\
\boldsymbol{V}^{*}
\end{array}\right\}
$$


In the above proposition, the components $V_{D}, V_{d}$ and $V_{s N}$ are respectively the components of $\mathcal{S}_{D}$, both components of particles in $\mathcal{P}_{d}$, and the normal component of particles in $\mathcal{P}_{s s}$ that have been suppressed in the construction of $M^{*}$ and $K^{*}$.

Proposition 6.

If (105) holds and if $\exists \lambda \in \mathbb{C}$ with $\operatorname{Re}(\lambda)>0$ the condition :

$$
\text { (iii) } \exists \boldsymbol{A}^{*} \in \mathbb{C}^{N^{*}}, \boldsymbol{A}^{*} \neq 0 \text {, such that }\left[\lambda^{2} M^{*}+K^{*}\right] \boldsymbol{A}^{*}=0 \text {; }
$$

is equivalent to conditions (i) and (ii) of proposition 2.

\section{f - Analysis of the various conditions for dynamic growth/instability}

\section{- Divergence}

\section{- Necessary condition}

When the masses are concentrated, $M$ is diagonal and $M_{S}^{*}=M^{*}$ is positive definite. The analysis of the conditions given in Proposition 4 reduces to the analysis of the eigenvalues of $K_{S}^{*}$. The existence of a negative eigenvalue of $K_{S}^{*}$ is a necessary (but not sufficient) condition for divergence.

\section{- Sufficient condition}

Proposition 5 shows that the existence of a positive real eigenvalue of the generalized eigenvalue problem $\left(\lambda^{2} M^{*}+K^{*}\right) \boldsymbol{V}^{*}=\boldsymbol{O}$ is a sufficient condition for divergence.

With both conditions, we have to check the compatibility of the associated eigenvector $\boldsymbol{V}^{*}$. In the present simplified context, it reduces to check the sign of the active tangential components :

$$
\sigma\left(V_{T p}^{*}\right)=-\sigma\left(r_{T p}^{0}\right) \text { for all } p \in P_{s s} .
$$

\section{- Flutter}

The existence of complex eigenvalues $\Lambda$ of the generalized eigenvalue problem $K^{*} \boldsymbol{A}^{*}=\Lambda M^{*} \boldsymbol{A}^{*}$ obviously implies the existence of a complex value $\lambda$ with a positive real part (since $\lambda^{2}=-\Lambda$ ) i.e. implies flutter. The analysis will focus on the modes of the structure that may be excited for the corresponding eigenvalue $\lambda$ (complex with a real part positive).

Note.

For divergence, the sufficient condition cannot be fulfilled before the necessary condition because :

- the smallest eigenvalue of $K_{S}^{*}$ is lower than or equal to the smallest real part of any eigenvalue of $K^{*}$,

- the smallest real part of any eigenvalue of $K^{*}$ is lower than or equal to any real eigenvalue of $K^{*}$ corresponding to a real compatible eigenvector $\boldsymbol{V}^{*}$. 


\subsection{Computation of the conditions and analysis}

\section{a - Construction of the matrices}

To check the stability along the quasistatic evolution, matrices $M^{*}, K^{*}, M_{S}^{*}, K_{S}^{*}$ have to be constructed at any change in the contact status.

Matrices $M^{*}$ and $K^{*}$ are constructed from the mass matrix $M$ and the stiffness matrix $K$ as previously shown (4.1.c) by removing specific equations and performing modifications (114) on specific tangential components.

Matrices $K^{*}$ and $M^{*}$ are therefore non symmetrical and depend on $\mu$.

\section{b - Computation of the lowest eigenvalue of $K_{S}^{*}$ (necessary condition for divergence)}

In order to check the necessary condition for divergence, we have to check whether $K_{S}^{*}$ is no longer definite positive. This is done by three different methods. Note that the same methods can be used to check if $M_{S}^{*}$ is positive when the mass matrix $M$ is consistent.

All methods give the same results :

1. minimizing the bilinear form $a^{*}(\mathbf{v}, \mathbf{v})$ under constraints (121) and $\|\mathbf{v}\|=1$ and checking that the minimum is negative,

2. applying Cholesky's method to $K_{S}^{*}$ and when it fails, $K_{S}^{*}$ is no longer positive definite,

3. computing the smallest eigenvalue of $K_{S}^{*}$, checking its sign and the compatibility (121) of the corresponding eigenvector.

Method 2 is a direct one and therefore the fastest and should be prefered when used for checking if $M_{S}^{*}$ is positive. Nevertheless, we use mostly the last method because it gives the eigenvector of $K_{S}^{*}$ corresponding to the smallest eigenvalue. This method consists in computing the smallest eigenvalue of $K_{S}^{*}$ (and not the one with smallest modulus, as usually done) :

- using the Power Method, we first compute the eigenvalue $\Lambda_{L}$ of $K_{S}^{*}$ with the largest modulus,

- if $\Lambda_{L}$ is negative, we have the desired result,

- if $\Lambda_{L}>0$, we shift all the eigenvalues towards the negative axis by constructing the modified matrix $K_{S}^{*}-\Lambda_{L} I$ (where $I$ denotes the matrix identity) and using the Power Method again, we compute the eigenvalue $\Lambda_{M}$ with the largest modulus of this modified matrix. The smallest eigenvalue $\Lambda_{m}$ of $K_{S}^{*}$ is then obtained as $\Lambda_{m}=\Lambda_{M}+\Lambda_{L}$ and we check its sign.

c - Computation of the generalized eigenvalue problem (sufficient condition for divergence, flutter)

In order to check the sufficient condition for divergence or for flutter, we have to determine all the eigenvalues of the generalized eigenvalue problem

$$
K^{*} \boldsymbol{V}^{*}=\Lambda M^{*} \boldsymbol{V}^{*} \quad\left(\Lambda=-\lambda^{2}\right) .
$$

A Lanczos algorithm (Rajakumar-Rogers (1991)) is used along with the double QR algorithm Ralston-Rabinowicz (1978).

The usual Lanczos method can be extended to non symmetric matrices (with real coefficients) and to generalized eigenvalue problems. It progressively constructs a non symmetrical Rayleigh matrix (tri-diagonal matrix), the order of which increases at every iterate. It is then possible to 
compute only a part of the spectrum in a given range (the eigenvalues with the smallest modulus in our case). The double QR method is then used for computing the eigenmodes and the eigenvectors of the tri-diagonal matrix .

Most of these computations were performed with the consistent mass matrix $M^{*}$. In order to speed up some of the computations for the most refined meshes, a diagonal mass matrix $M$ was used, which also leads to a diagonal matrix $M^{*}$.

\section{d - Synthesis of the analysis of the dynamic growth/instability conditions}

Let us now summarize how to check the various conditions given in this section.

\section{- Necessary condition for divergence}

Here, we only have to check whether matrix $K_{S}^{*}$ (symmetric) has a negative eigenvalue.

- Sufficient condition for divergence

We just have to check whether the generalized eigenvalue problem (122) has any real negative eigenvalue $\Lambda$ which corresponds to real $\lambda= \pm \sqrt{-\Lambda}$. With the problems studied so far, we have never observed this situation.

\section{- Sufficient condition for flutter}

Unless all the eigenvalues $\Lambda$ of the generalized eigenvalue problem (122) are real, a complex $\lambda$ with a positive real part always exists (keeping in mind that $\Lambda=-\lambda^{2}$ ). It is likely that significant consequences will arise only when some lower mode is affected by flutter and when the growth rate (the real part of the complex eigenvalue) is large enough.

Moreover, it can be noted that :

- with the necessary condition for divergence, the compatibility condition (121) has always been satisfied by the computed eigenvectors,

- matrices $M^{*}$ and $M_{S}^{*}$ were never found to be singular ( $M_{S}^{*}$ was always positive definite) in all the computations involving finite element discretizations performed in the course of this study.

\subsection{Application to the block sliding on a plane}

In this section, stability analysis is conducted on the example of a block of Polyurethane sliding on an Araldite plane. The quasistatic solution of this problem was given in Section 1. The following computations were conducted by S. Barbarin (see Barbarin (1997) and Martins et al (1999)). Experiments performed by T. Zeghloul and B. Villechaise (see Zeghloul-Villechaise (1996)) showed that jumps occur in both the tangential displacement and the friction force during the sliding. Stress waves were also observed experimentally when jumps occurred. In Section 1, a model of the experiment was presented and the various steps of the quasistatic solution were given. In the present section, the analysis of the stability is conducted along these various steps of the solution in order to check the possible occurrence of an instability.

\section{a - Analysis of the eigenvalues}

We examine the solution (on the mesh of Fig. 4 when the prescribed tangential displacement $U_{T}$ gradually increases (see Fig. 3). Construction of all matrices and computation of the eigenvalues of $K_{S}^{*}$ and $M^{*-1} K^{*}$ are performed only when a change occurs in the contact condition (sticking, 
sliding or separate nodes). In Table 3, at each value of the prescribed tangential displacement $U_{T}$ (the applied velocity is constant and equal to $0.103 \mathrm{~mm} / \mathrm{s}$, a first period of $15 \mathrm{~s}$ is dedicated to the normal loading), the total number of stick, slip or no-contact nodes is given together with the minimum real eigenvalues of $K_{S}^{*}$ and $M^{*-1} K^{*}$ and the lowest mode number corresponding to a complex eigenvector of $M^{*-1} K^{*}$.

The results given in Table 3 show that :

- the necessary condition for divergence is first satisfied at $U_{T}=-1.35 \mathrm{~mm}$ when a second node begins to slide,

- the sufficient condition for divergence is never satisfied,

- flutter occurs first for very high modes. Flutter occurs for a low order mode (the 3rd mode) exactly when the necessary condition for divergence is first satisfied at $U_{T}=-1.35 \mathrm{~mm}$. In other examples, it was found to occur nearly at the same time or a little earlier. Table 3 also shows that the steady sliding state reached at the end of the theoretical quasistatic solution is still unstable by flutter (see the last row in the relevant table).

\begin{tabular}{|c|c|c|c|c|c|c|c|c|}
\hline Time & $U_{T}$ & \multicolumn{2}{|c|}{ Contact Nodes } & \multicolumn{2}{|c|}{ Min Real E.V. of } & Flutter & Experimental \\
\cline { 3 - 8 }$(\mathrm{s.})$ & $(\mathrm{mm})$ & Stick & Slip & Separ. & $K_{S}^{*}$ & $M^{*-1} K^{*}$ & (Mode) & data \\
\hline 23.4 & -0.87 & 20 & 1 & 0 & 0.076 & $8.1910^{6}$ & 57 & - \\
\hline 25.2 & -1.05 & 19 & 1 & 1 & 0.071 & $7.9110^{6}$ & 11 & - \\
\hline 26.6 & -1.20 & 18 & 1 & 2 & 0.068 & $7.6910^{6}$ & 10 & - \\
\hline 28.1 & -1.35 & 17 & 2 & 2 & $\mathbf{- 0 . 1 3 0}$ & $7.4310^{6}$ & $\mathbf{3}$ & - \\
\hline 29.1 & -1.45 & 16 & 2 & 3 & $\mathbf{- 0 . 1 0 6}$ & $7.0910^{6}$ & $\mathbf{3}$ & - \\
\hline 31.0 & -1.65 & 14 & 4 & 3 & $\mathbf{- 0 . 5 0 2}$ & $6.2610^{6}$ & $\mathbf{3}$ & - \\
\hline 32.0 & -1.75 & 12 & 6 & 3 & $\mathbf{- 0 . 6 7 6}$ & $5.4710^{6}$ & $\mathbf{3}$ & - \\
\hline 32.3 & -1.78 & 10 & 8 & 3 & $\mathbf{- 0 . 7 6 0}$ & $6.7610^{6}$ & $\mathbf{3}$ & - \\
\hline 32.7 & -1.82 & 7 & 11 & 3 & $\mathbf{- 0 . 8 2 1}$ & $5.3710^{6}$ & $\mathbf{3}$ & - \\
\hline 34.4 & -2.00 & 4 & 14 & 3 & $\mathbf{- 0 . 8 5 1}$ & $3.3410^{6}$ & $\mathbf{3}$ & \\
\hline 36.4 & -2.20 & 3 & 15 & 3 & $\mathbf{- 0 . 8 5 8}$ & $3.0810^{6}$ & $\mathbf{3}$ & $1^{\text {st }}$ small jump \\
\hline 38.8 & -2.45 & 2 & 16 & 3 & $\mathbf{- 0 . 8 6 4}$ & $2.7810^{6}$ & $\mathbf{3}$ & - \\
\hline 41.3 & -2.71 & 2 & 16 & 3 & $\mathbf{- 0 . 8 6 4}$ & $2.7810^{6}$ & $\mathbf{3}$ & $2^{\text {nd }}$ jump \\
\hline 41.7 & -2.75 & 2 & 15 & 4 & $\mathbf{- 0 . 8 5 9}$ & $2.6810^{6}$ & $\mathbf{5}$ & - \\
\hline 48.0 & -3.40 & 1 & 16 & 4 & $\mathbf{- 0 . 8 6 8}$ & $2.3010^{6}$ & $\mathbf{5}$ & - \\
\hline 75.2 & -6.20 & 0 & 17 & 4 & $\mathbf{- 0 . 9 7 4}$ & $1.2310^{6}$ & $\mathbf{5}$ & - \\
\hline
\end{tabular}

Table 3. Results of the stability analysis and comparison with experimental measurements.

\section{b - Analysis of the eigenmode shapes}

In this section, we plot the shape of the eigenvectors associated with the various fulfilled conditions shown in Table 3.

\section{Modes associated with the negative real eigenvalue of $K_{S}^{*}$}

The eigenvectors associated with this negative eigenvalue of $K_{S}^{*}$ are plotted in Fig. 25. They give a direction in which an unstable evolution would be energetically admissible, i.e. an evolution with loss of energy into some external sink. These mode shapes are distinctly reminiscent of the 


$$
U_{T}=-0.87 \mathrm{~mm}
$$

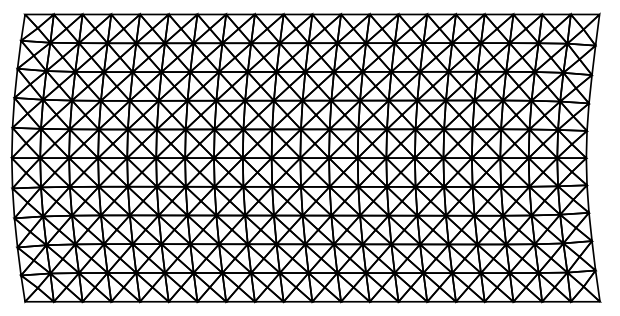

$$
U_{T}=-1.55 \mathrm{~mm}
$$

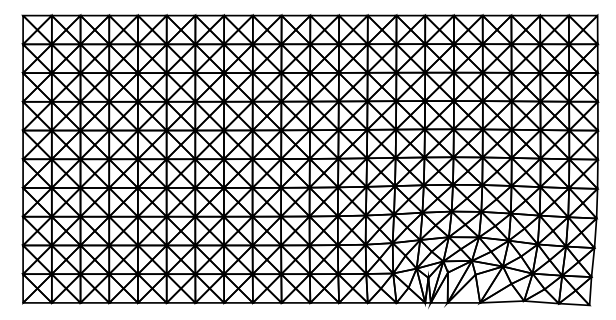

$U_{T}=-1.35 \mathrm{~mm}$

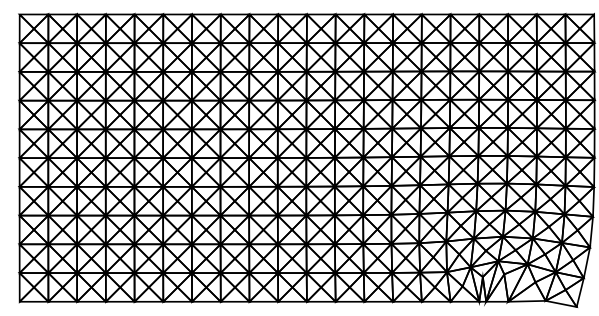

$U_{T}=-1.78 \mathrm{~mm}$

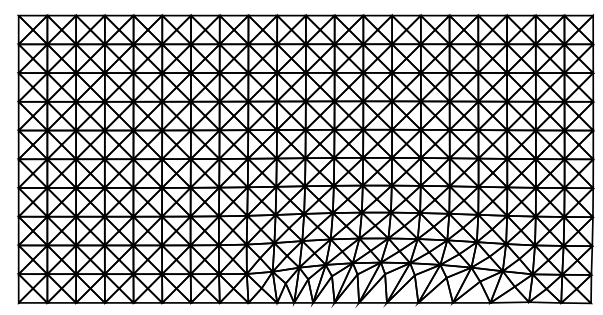

Figure 25. Eigenvector associated with the smallest eigenvalue of $K_{S}^{*}$ for various values of $U_{T}$.

waves observed experimentally in the Polyurethane block when a jump occurs. However, as can be seen in Table 3, the sufficient condition that would guarantee the occurrence of a divergence growth of the dynamic solution (a negative eigenvalue of $M^{*-1} K^{*}$ ) is in fact never satisfied.

\section{Modes associated with the flutter}

In Fig. 26, we have plotted the shapes of the eigenvectors associated with the lowest mode which can be excited by flutter, and that at various values of the prescribed displacement of the support. The deformation of the mesh represents the real part of the direction of the eigenvector and the arrows, the real part of its derivative (the directions were computed with $M^{*-1} K^{*}$ ), for two values of $\mathrm{U}_{T}:-0.87 \mathrm{~mm}$ (high mode, 57 ) and $-1.4 \mathrm{~mm}$ (low mode, 3 ). 


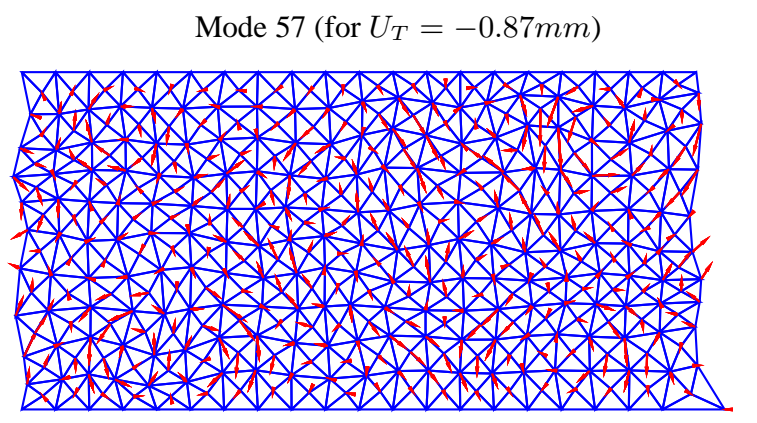

Mode 3 (for $U_{T}=-1.4 \mathrm{~mm}$ )

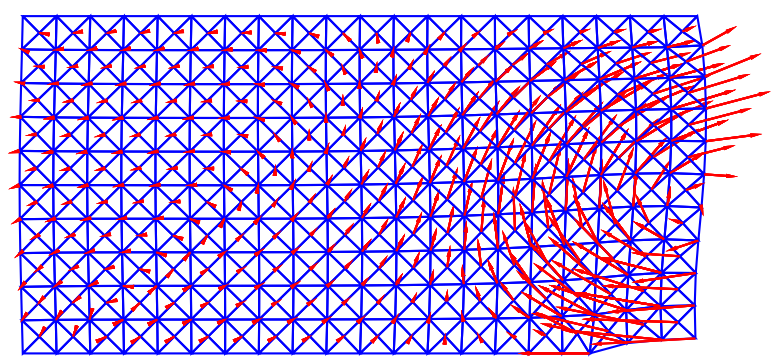

Figure 26. Eigenmode corresponding to the lowest flutter frequency for various values of $U_{T}$.

\section{c - Influence of the mass on the stability}

Fig. 27 gives the evolution of the lowest eigenvalue of $M^{*-1} K^{*}$ when the displacement prescribed on the plate increases. The sufficient condition for divergence instability is that $\Lambda_{\text {min }}$ becomes negative. In this case, the criterion is never fulfilled, but the sharp decrease in $\Lambda_{\min }$ is worth noting. In Fig. 27, the analysis is conducted on the three following cases :

- case $1: M^{*}$ is taken to denote the identity matrix (criterion then based only on the stiffness matrix),

- case 2 : diagonalization of the mass matrix by concentrating of the consistent mass matrix,

- case 3 : consistent mass matrix.

As it could be expected, the various choices have no significant influence on the evolution of $\Lambda_{\min }$, and actually, they have no influence on the transition to instability at $\Lambda_{\min }=0$. We can therefore use either case 1 or case 2, where the computations are easier (diagonal matrix).

When dealing with the sufficient condition for flutter, we considered two cases : the diagonal mass matrix and the consistent mass matrix. In Fig. 28, the evolution of the modes excited by flutter when the prescribed displacement of the plate increases is given in both cases. In Fig. 29, the spectrum of the flutter eigenvalues is given at a given step of the loading in both cases. It can be observed that the choice of the mass matrix has an effect on the high frequency modes. When performing the flutter analysis, we are interested in the low frequency modes and the use of a diagonal mass matrix is therefore again recommended. 


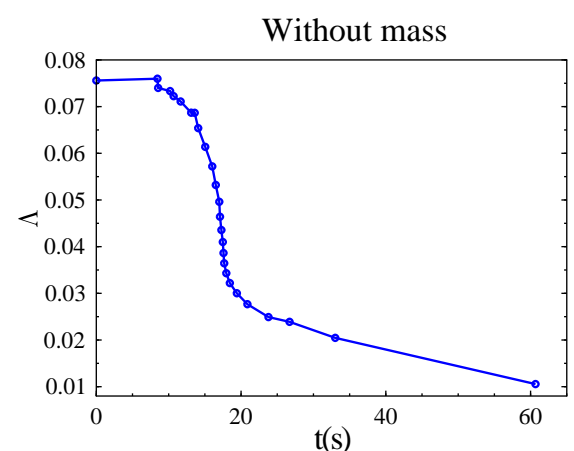

$\mathrm{t}(\mathrm{s})$

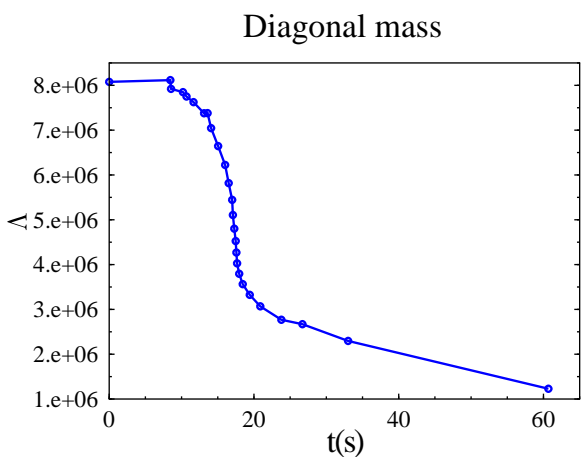

$\mathrm{t}(\mathrm{s})$

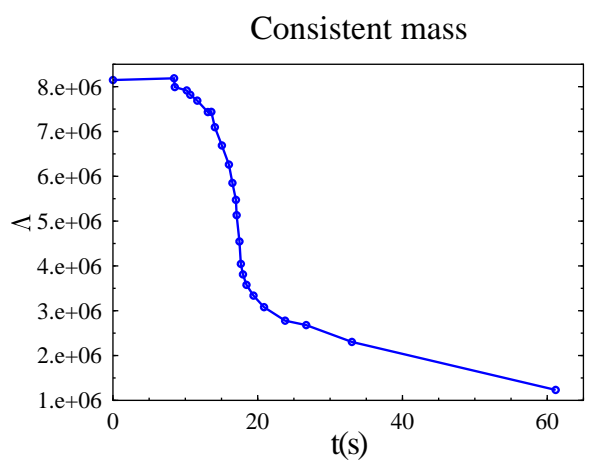

Figure 27. Comparison between the sufficient condition for divergence (the lowest eigenvalue of $M^{*-1} K^{*}$ ) with three choices of mass matrix.

Diagonal mass

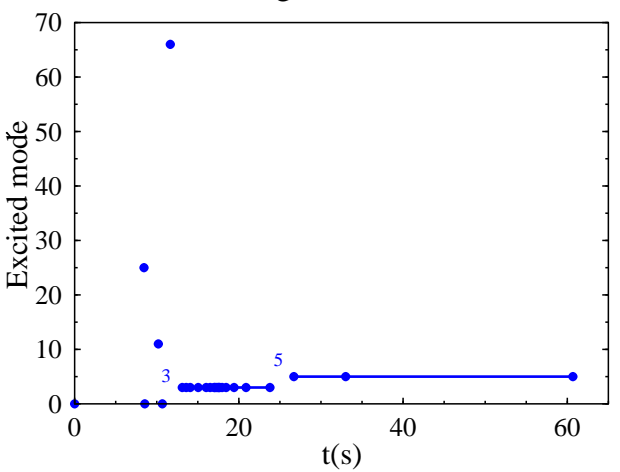

Consistent mass

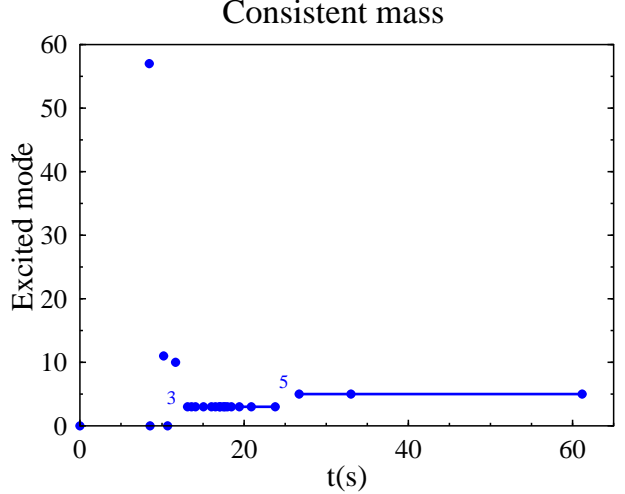

Figure 28. Effects of mass on the flutter modes during the loading. 


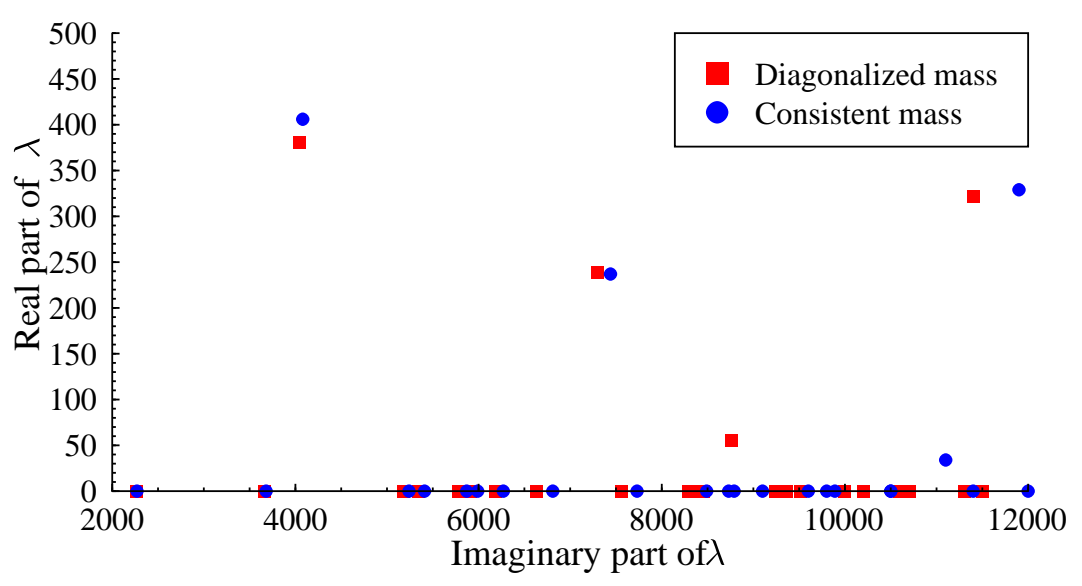

Figure 29. Effect of mass on the first 25 flutter modes.

\section{d - Effects of the mesh}

The various criteria were computed for the various meshes presented in Fig. 30.

\section{Effects of the mesh on the necessary and on the sufficient condition for divergence}

The evolution of the lowest eigenvalue of $K_{S}^{*}$ is given in Fig. 31. The evolution of the frequency of the the first flutter mode (eigenvalue of $M^{*-1} K^{*}$ ) is given in Fig. 32. These results show that with a given shape of the mesh, the refinement has no effect, but that a slight change in the eigenvalues is observed when the finite element shape is changed.

\section{Effects of the mesh on the sufficient condition for flutter}

Fig. 33 gives, with the various meshes, the number of the lowest flutter mode which can be excited when the loading increases. Fig. 34 gives the total spectrum for two meshes (15 and 25 contact nodes) at a given loading step. It can be seen that the mesh refinement influences the capture of the modes and that the mesh acts more or less as a filter. It can be seen from Fig. 33, that the mode 5 is caught sooner when the mesh is more refined. In Fig. 34, when the mesh has been refined (15 and 25 contact nodes), it can be seen that the frequencies of the lowest flutter mode (mode 3) are the same, although a difference occurs in the growth rate, and, on the contrary, the higher modes are different. That means that the mesh has to be sufficiently refined in order to catch the lowest modes associated with the frequencies involved in the mechanical phenomena under investigation. 


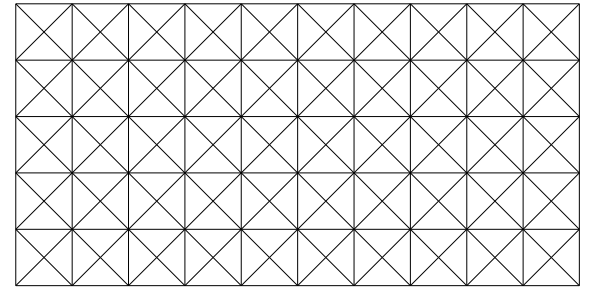

232 dof

$11 \mathrm{cn}$

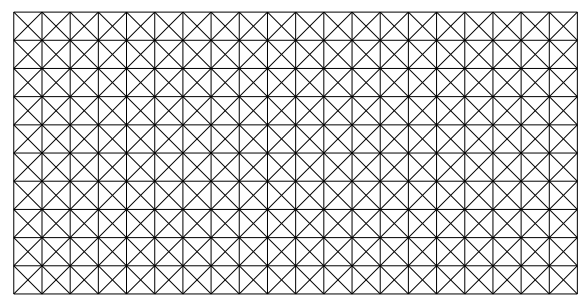

862 dof

$21 \mathrm{cn}$

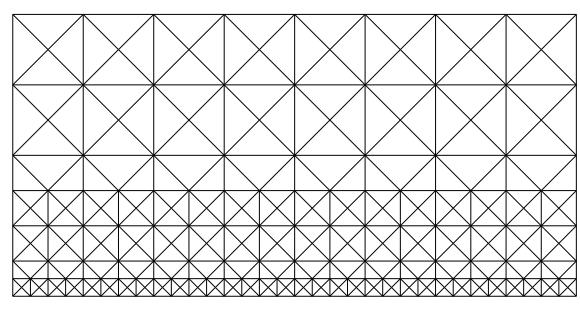

448 dof

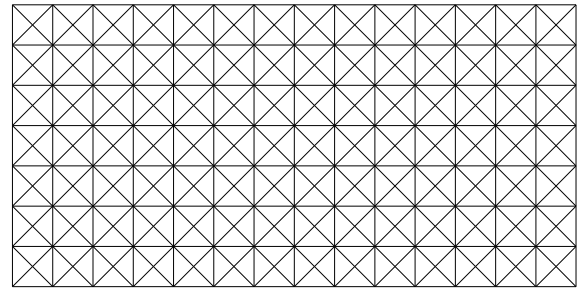

536 dof

$15 \mathrm{cn}$

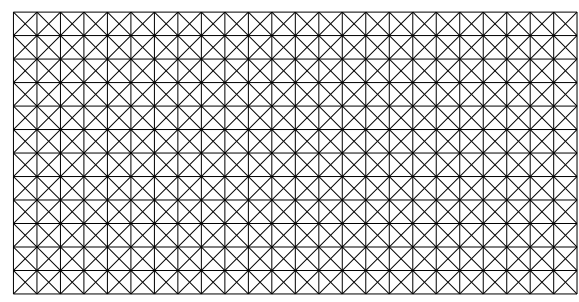

1226 dof

$25 \mathrm{cn}$

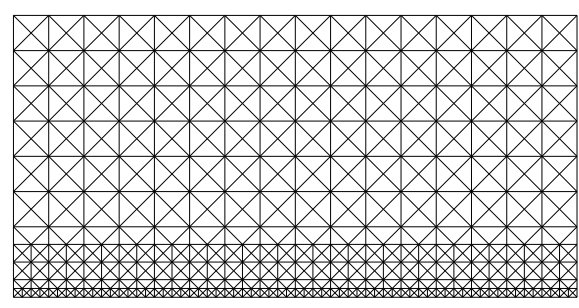

1144 dof $\quad 65 \mathrm{cn}$

Figure 30. The various meshes. 

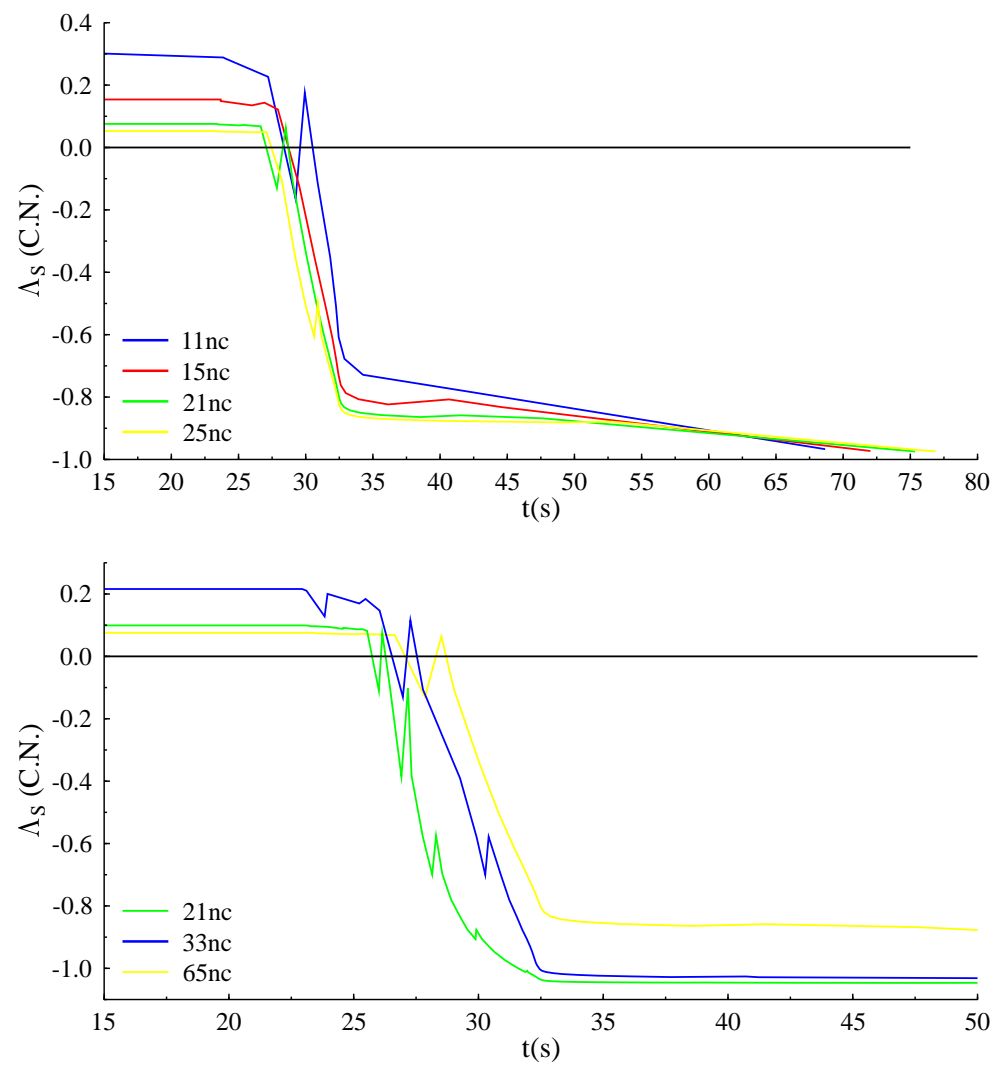

Figure 31. Influence of mesh refinement on the necessary condition for divergence.
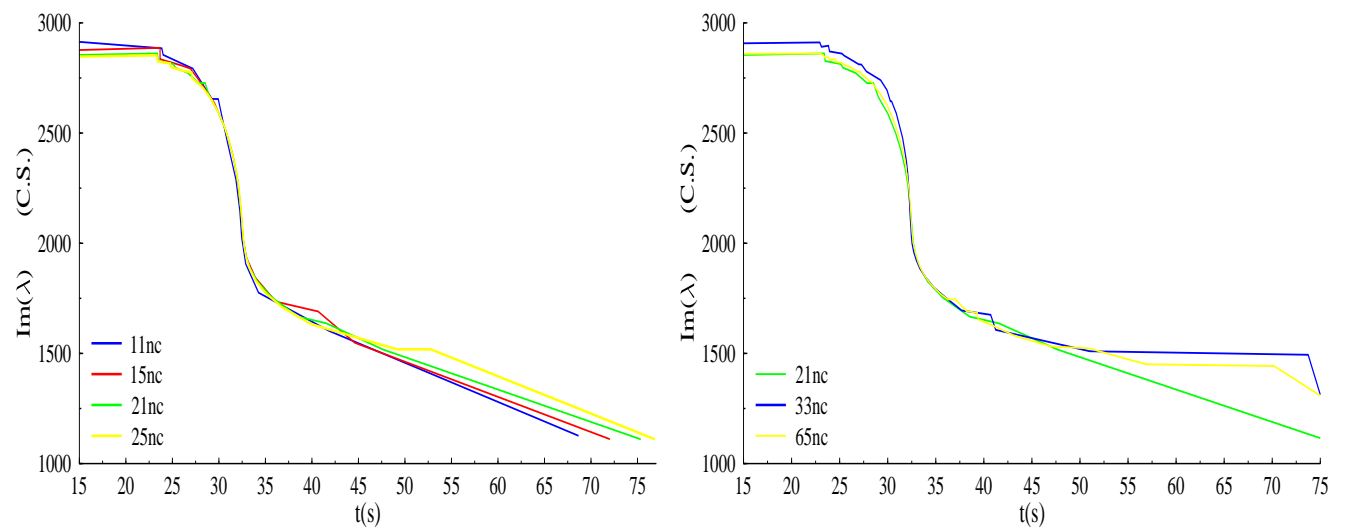

Figure 32. Influence of mesh refinement on the sufficient condition for divergence. 

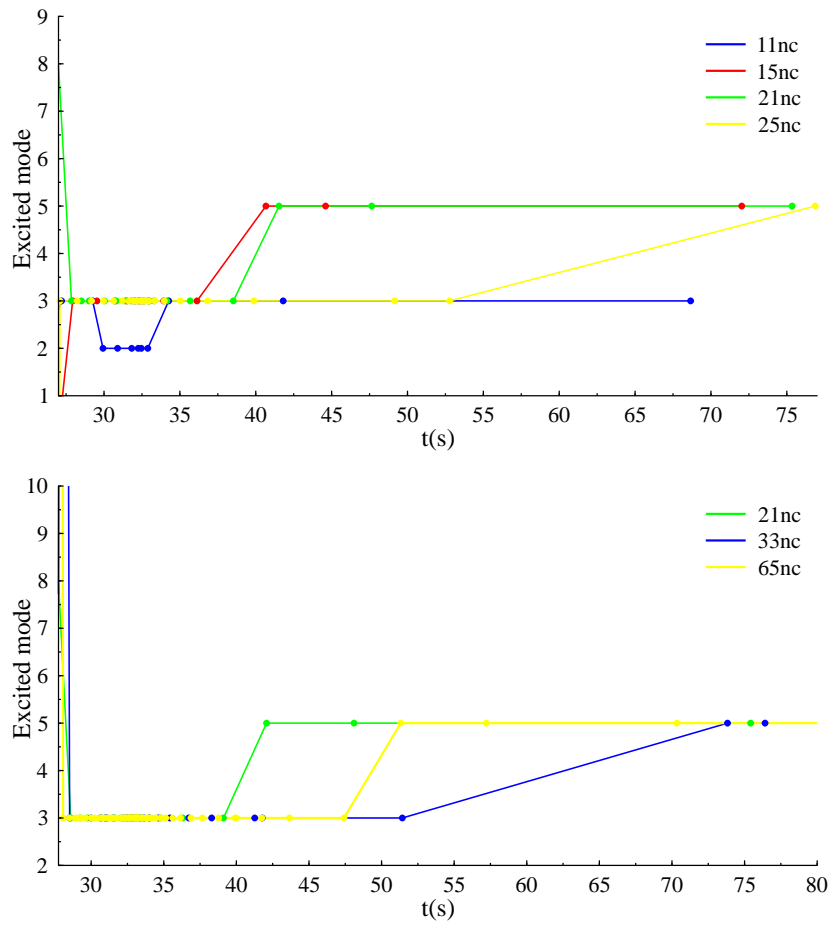

Figure 33. Influence of mesh refinement on the sufficient condition for flutter.

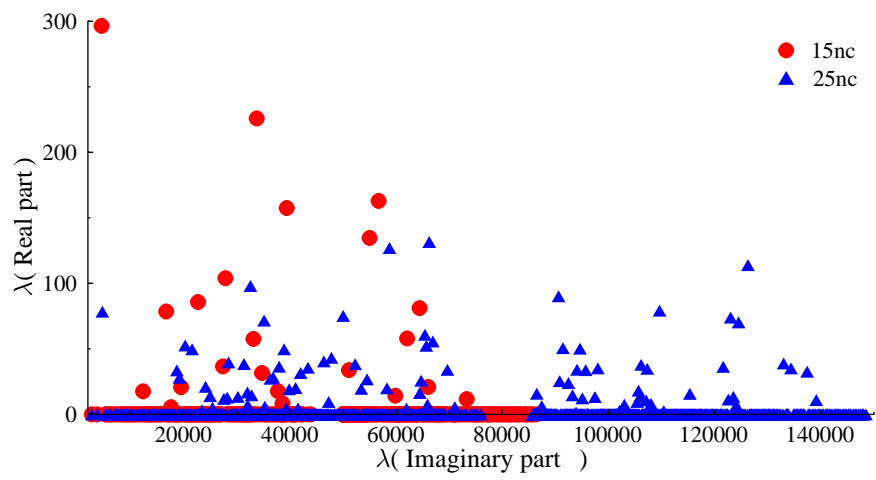

Figure 34. Influence of mesh refinement on the flutter : the complete spectrum at a given loading step. 


\section{e - Comparison with experimental results}

In Fig. 35, the changes with respect to time in both the experimental and theoretical tangential forces are plotted. On the theoretical curve, the dot indicates the time at which the necessary condition for divergence instability and the sufficient condition for growing flutter-type oscillations are first satisfied.

These conditions are satisfied earlier than the first experimental jumps observed : both conditions are satisfied at $U_{T}=-1.35 \mathrm{~mm}$, while the first experimental small jump is observed at $U_{T}=-$ $2.2 \mathrm{~mm}$, and a second jump is observed at $U_{T}=-2.71 \mathrm{~mm}$ (see also Table 3). In the following section, we present a preliminary study on the possible effects of viscous damping on the stability analysis.

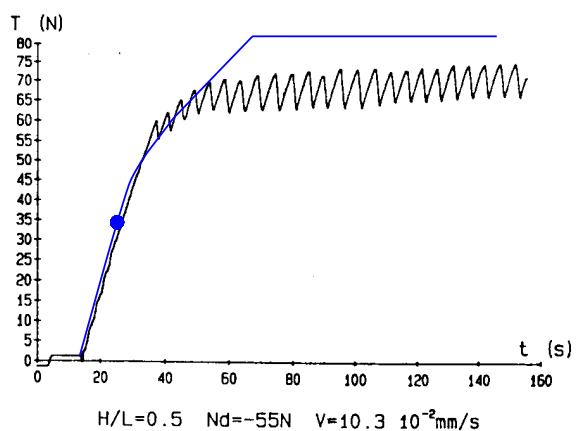

Figure 35. Variations with respect to time of the experimental and theoretical total tangential forces.

\section{f - Effects of viscous damping}

As seen above, flutter occurs too early in the model in comparison with the experiment. A simplified way of introducing viscous damping effects into the problem is to consider a damping matrix of the Rayleigh type $\alpha M+\beta K$ in the dynamic equations of motion and the dynamic stability analysis. In this manner, it is possible to delay the flutter occurrence (and even to eliminate it with a sufficiently strong damping effect).

The frequencies of the first 18 natural modes of the structure range between $454 \mathrm{~Hz}$ and $1530 \mathrm{~Hz}$ in the initial phase of elastic behaviour, i.e., when all the nodes are stuck. The damping ratio $\xi$ is defined as $\frac{-\operatorname{Re}(\lambda)}{|\operatorname{Im}(\lambda)|}$, where $\lambda$ is the eigenvalue solution of (123), when all the contact nodes are stuck. It depends on the frequencies and is known to be decreasing in that range in the case of Polyurethane. We therefore compute several values of $\alpha$ and $\beta$ (see Clough-Penzien (1975)) so as to obtain the various decreasing evolutions of the damping ratio given in Fig. 36. Stability has been studied with six of these different damping levels (characterized by the value of $\xi$ at $454 \mathrm{~Hz}$ given in Fig. 36). The eigenvalues are now computed from :

$$
\left(\lambda^{2} M^{*}+\lambda\left(\alpha M^{*}+\beta K^{*}\right)+K^{*}\right) \boldsymbol{V}^{*}=0 .
$$


The values of the imposed tangential displacement $U_{T}$ at which flutter occurs are given on Fig. 37 for the six damping levels. The sudden change in $U_{T}$ occurring between the values of $\xi=0.1475$ and $\xi=0.15$ on Fig. 37 is due to the change in the excited mode.

The first value corresponds to mode 3 and the second one to mode 5 . This means that when the damping is strong enough, mode 3 is no longer excited by flutter.

It can therefore be seen from Fig. 37 that the occurrence of instability is greatly delayed by the introduction of viscous damping. Flutter mode 3 appears at $U_{T}=-1.35 \mathrm{~mm}$ in the no-viscosity case and only at $U_{T}=-6.2 \mathrm{~mm}$ with the maximum damping level considered here. To assess the occurrence of flutter more exactly when viscosity is taken into account, it would be necessary to measure exactly the loss angle of the material in the appropriate frequency range.

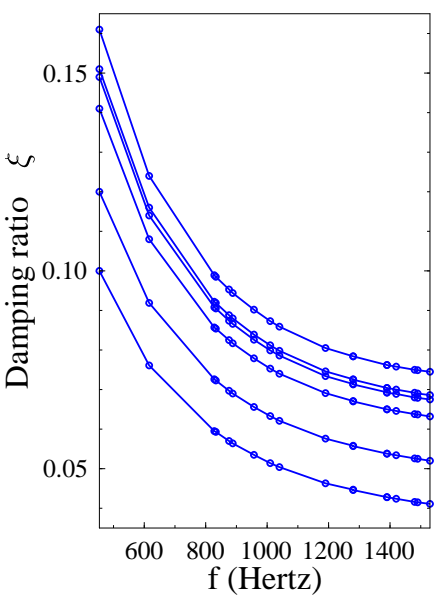

Figure 36. Frequency dependence of the damping ratios obtained for the six values of $\alpha$ and $\beta$ considered.

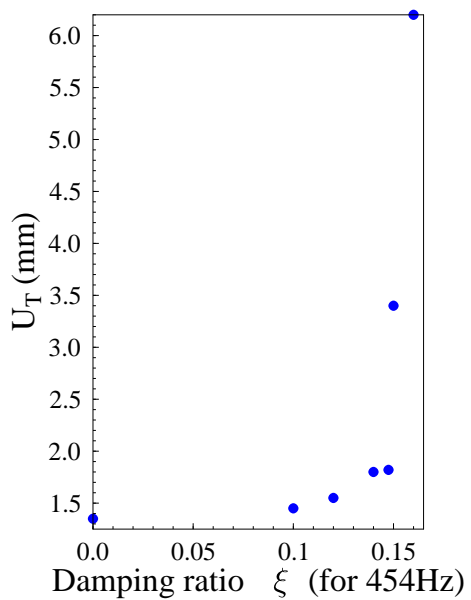

Figure 37. Prescribed tangential displacement at which a low order flutter mode occurs for the various damping levels given in Fig. 36.

\section{g - Conclusions on the analysis of the block sliding on a plane}

The steady sliding solution of the block is found to be unstable by flutter. During the tangential quasistatic loading process, we observe that :

- the necessary condition for divergence is satisfied but it occurs earlier than the first experimental jump, and the corresponding eigenmode suggests the onset of the waves experimentally observed,

- the sufficient condition for divergence is never satisfied,

- growing flutter-type oscillations occur here for the lower order modes at the same time as the necessary condition for divergence is satisfied,

- introducing damping into the stability analysis delays the occurrence of flutter and may give better agreement with the experimental results. A hyper-visco-elastic model is suggested for the behaviour of the Polyurethane. 


\section{Stability analysis of a steady sliding solution in finite deformations}

We will first recall the main results of the stability analysis for hyperelastic finite dimensional problems given in Vola-Raous-Martins (1999), Martins-Pinto da Costa (2000) and Vola-Pinto da Costa-Barbarin-Martins-Raous (1999). Here, we will focus on the numerical discretization and the computation of the various conditions as well as the flutter solutions. An application to the industrial problem of a waist seal sliding on a car window will be presented.

As in the previous section, the results given here are based on a property which looks like that of Liapunov, stating that instability of the linearized system implies that of the nonlinear one. But, in the present problem, such a property had to be proved because the problem is not differentiable, and Liapunov's results no longer applies.

It should be noted that it is more simple to study the instability of steady sliding than to investigate the one of the complete quasistatic solutions (see Martins-Pinto da Costa (2000)) : in the present case, the nodes are either sliding or separate and the friction force is $r_{T}=\mu r_{N} \operatorname{sgn}\left(v_{T}\right)$.

\subsection{The discrete dynamics problem}

As described above in Section 3, the discrete dynamic problem can be written as :

$$
\begin{array}{r}
\widetilde{M} \widetilde{\boldsymbol{u}}+\widetilde{\boldsymbol{F}}_{\text {int }}(\mathbf{u})=\widetilde{\boldsymbol{F}}_{\text {ext }}+\mathbf{r}, \\
\forall \mathbf{r}_{n}^{*} \in \mathcal{C}_{n}^{h} \quad\left(\mathbf{r}_{n}^{*}-\mathbf{r}_{n}\right)^{T} \boldsymbol{\omega}_{n}(\mathbf{u}) \geq 0, \\
\forall \mathbf{r}_{t}^{*} \in \mathcal{C}_{t}^{h}\left(\mathbf{r}_{n}\right) \quad\left(\mathbf{r}_{t}^{*}-\mathbf{r}_{t}\right)^{T} \dot{\boldsymbol{\omega}}_{t}(\dot{\boldsymbol{u}}) \geq 0,
\end{array}
$$

where :

- $\widetilde{M}=H M H^{T}, \widetilde{\boldsymbol{F}}_{\text {int }}=H \boldsymbol{F}_{\text {int }}, \widetilde{\boldsymbol{F}}_{\text {ext }}=H \boldsymbol{F}_{\text {ext }}, \widetilde{\ddot{\boldsymbol{u}}}=H \ddot{\boldsymbol{u}}$ and $\widetilde{\boldsymbol{u}}=H \mathbf{u}$,

- $\boldsymbol{\omega}_{n}(\boldsymbol{u})$ is the current gap and, $\dot{\boldsymbol{\omega}}_{t}(\dot{\boldsymbol{u}})$ and $\dot{\omega}_{n_{i}}(\mathbf{v})=H_{n_{i}} \mathbf{v}$ are the current relative tangential and normal velocity ( $\boldsymbol{G}$ is the initial distance in the reference configuration) :

$$
\omega_{n_{i}}(\mathbf{u})=H_{n_{i}} \mathbf{u}+G_{i}, \quad \dot{\omega}_{t_{i}}(\dot{\boldsymbol{u}})=H_{t_{i}} \dot{\boldsymbol{u}}-v_{o b s} \quad \forall i \in 1, . ., n_{c},
$$

- and where $H, \mathcal{C}_{n}^{h}$ and $\mathcal{C}_{t}^{h}\left(\boldsymbol{r}_{n}\right)$ are defined by Eq. (62) and Eq. (64) of Section 3.2

\subsection{Instability of the steady sliding solution}

\section{a - Notations}

In the case of a steady sliding solution, the problem is simpler. The contact nodes are :

- either in sliding contact $\left(r_{n_{i}}^{0}>0, u_{n_{i}}^{0}+G_{i}=0, r_{t_{i}}^{0}=\mu \operatorname{sgn}\left(v_{o b s}\right) r_{n_{i}}^{0}\right.$ and $\left.\dot{u}_{t_{i}}^{0} \neq v_{o b s}\right)$,

- or strictly separated $\left(r_{t_{i}}^{0}=r_{n_{i}}^{0}=0\right.$ and $\left.u_{n_{i}}^{0}+G_{i}>0\right)$,

where $\square^{0}$ denotes the value of $\square$ in the steady sliding equilibrium configuration.

Linearization assumption : we assume that it is possible to find perturbed dynamic solutions in the neighborhood of this steady sliding state, such that the contact state of each contact candidate node is preserved ; i.e. each node in sliding contact remains in sliding contact $\left(u_{n_{i}}+G_{i}=\dot{u}_{n_{i}}=0, r_{t_{i}}=\mu \operatorname{sgn}\left(r_{t_{i}}^{0}\right) r_{n_{i}}\right.$ and $\left.\dot{u}_{t_{i}} \neq v_{o b s}\right)$, and each node not in contact 


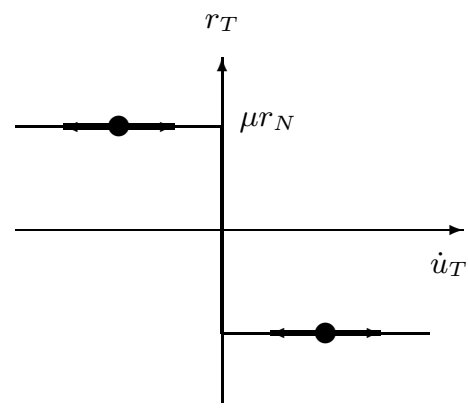

Figure 38. Linearization assumption presented on the graph of the Coulomb law (case where $v_{o b s}=0$ ).

remains so with $\left(r_{t_{i}}=r_{n_{i}}=0\right.$ and $u_{n_{i}}+G_{i}>0$ ) (see Fig. 38). We use the subscript $f$ (free) to denote the degrees of freedom corresponding to the displacement components of nodes which are not concerned by the contact or contact nodes not currently in contact. The subscripts $n$ (respectively $t$ ) denote those that correspond to normal (respectively tangential) displacements of sliding nodes. The dynamical problem can be then written :

$$
\left[\begin{array}{lll}
M_{f f} & M_{f n} & M_{f t} \\
M_{n f} & M_{n n} & M_{n t} \\
M_{t f} & M_{t n} & M_{t t}
\end{array}\right]\left[\begin{array}{l}
\ddot{\mathbf{u}}_{f} \\
0 \\
\ddot{\mathbf{u}}_{t}
\end{array}\right]+\left[\begin{array}{l}
\boldsymbol{F}_{\text {int }_{f}}\left(\mathbf{u}_{f}, \mathbf{u}_{t}\right) \\
\boldsymbol{F}_{\text {int }_{n}}\left(\mathbf{u}_{f}, \mathbf{u}_{t}\right) \\
\boldsymbol{F}_{\text {int }_{t}}\left(\mathbf{u}_{f}, \mathbf{u}_{t}\right)
\end{array}\right]=\left[\begin{array}{l}
0 \\
\boldsymbol{r}_{n} \\
\mu s \boldsymbol{r}_{n}
\end{array}\right],
$$

where $s=\operatorname{sgn}\left(v_{\text {obs }}\right)$.

Using the equations of motion corresponding to the normal degrees of freedom of the sliding nodes $(n)$ to eliminate the non vanishing reactions, the equations that govern the smooth dynamic evolution of the structure in the neighborhood are :

$$
\underbrace{\left[\begin{array}{ll}
M_{f f} & M_{f t} \\
M_{t f}^{*} & M_{t t}^{*}
\end{array}\right]}_{M^{*}}\left[\begin{array}{l}
\ddot{\boldsymbol{u}}_{f} \\
\ddot{\boldsymbol{u}}_{t}
\end{array}\right]+\left[\begin{array}{l}
\boldsymbol{F}_{\text {int }_{f}}\left(\mathbf{u}_{f}, \mathbf{u}_{t}\right) \\
\boldsymbol{F}_{\text {int }_{t}}^{*}\left(\mathbf{u}_{f}, \mathbf{u}_{t}\right)
\end{array}\right]=\left[\begin{array}{l}
\mathbf{0} \\
\mathbf{0}
\end{array}\right],
$$

where $\boldsymbol{F}_{\text {int }_{t}}^{*}=\boldsymbol{F}_{\text {int }_{t}}-\mu s \boldsymbol{F}_{\text {int }_{n}}$ and $M_{t \square}^{*}=M_{t \square}-\mu s M_{n \square}$. If the matrix $M^{*}$ is non-singular, the second order differential system (129) is regular and the normal displacements of the free contact candidate nodes, as well as the tangential velocities and the normal reactions of the nodes in sliding contact, will depend continuously on the initial conditions and on time. Consequently, in a sufficiently small neighborhood of the steady sliding state, the solution of (129) and (128) for perturbed initial conditions arbitrarily close to $\boldsymbol{u}_{f}^{0}, \boldsymbol{u}_{t}^{0}$ and $\dot{\boldsymbol{u}}_{f}^{0}=\dot{\boldsymbol{u}}_{t}^{0}=\mathbf{0}$ leads to a "smooth" solution of the general system (130) to (133).

$$
\begin{gathered}
M d \dot{\boldsymbol{u}}+\boldsymbol{F}_{\text {int }}(\mathbf{u}) d \tau-\boldsymbol{F}_{\text {ext }} d \tau-H^{T} \mathbf{r} d \nu=\mathbf{0}, \\
\int_{] a, b]} d \dot{\boldsymbol{u}}=\dot{\boldsymbol{u}}^{+}(b)-\dot{\boldsymbol{u}}^{+}(a)
\end{gathered}
$$




$$
\begin{gathered}
\forall \boldsymbol{r}_{n}^{*} \in C_{n}^{h} \quad\left(\boldsymbol{r}_{n}^{*}-\boldsymbol{r}_{n}\right)^{T}\left(H_{n} \boldsymbol{u}+\boldsymbol{G}\right) \geq 0, \\
\forall \boldsymbol{r}_{t}^{*} \in C_{t}^{h}\left(\boldsymbol{r}_{n}\right) \quad\left(\boldsymbol{r}_{t}^{*}-\boldsymbol{r}_{t}\right)^{T}\left(H_{t} \dot{\boldsymbol{u}}-\boldsymbol{v}_{o b s}\right) \geq 0 .
\end{gathered}
$$

The equations of motion (129) are linearized :

$$
M^{*} \delta \ddot{\boldsymbol{u}}^{*}(t)+K^{*} \delta \mathbf{u}^{*}(t)=\mathbf{0},
$$

where :

- $\delta \mathbf{u}^{*}(t)$ denotes a small increment in the active displacements,

- $K^{*}$ is a non-symmetric matrix with dimension $N^{*}(\leq N)$ constructed from the stiffness matrix in the same way as $M^{*}$ (see 129). $K^{*}$ depends on the friction coefficient.

\section{b - Stability analysis}

The important point is that if the steady sliding equilibrium state is dynamically unstable for the linearized system (134), it will also be unstable for the non-linear system (129).

The analysis of the dynamic stability of the system (134) leads, as it was shown in Section 4, to the generalized eigenvalue problem :

$$
\left(\lambda^{2} M^{*}+K^{*}\right) \boldsymbol{V}^{*}=0
$$

The occurrence of a non-trivial eigenvector $V^{*}$ for some $\lambda$ with a strictly positive real part implies the instability of the steady sliding state. It will be :

- a divergence instability if $\operatorname{Im}(\lambda)=0$ (non-oscillatory),

- a flutter instability if $\operatorname{Im}(\lambda) \neq 0$ (oscillatory).

\section{c - Computation of the eigenvalue spectrum}

As previously noted, $M^{*}$ and $K^{*}$ are non symmetric. Their dimension $N^{*}$ is large (only a few equations were eliminated in (129)). We therefore have to compute the whole spectrum of the complex eigenvalues of the generalized eigenvalue problem (135). This problem is solved using the Lanczos algorithm (see Rajakumar-Rogers (1991)) along with the double QR method.

\subsection{Discussion of the stability analysis on a simplified waist seal geometry (presented in Section 3.6)}

In this section, the stability analysis is conducted on the simplified waist seal geometry presented in Section 3 (see Fig. 18) : on one hand, the eigenvalue spectrum characterizing the flutter instabilities is computed, on the other hand, the flutter oscillations including normal shocks and stick-slip are computed using the dynamic formulation given in Section 3. The influence of various parameters on the stability analysis will also be presented. The computations were carried out by D. Vola (Vola (1998)). 


\section{a - General comments on the stability analysis}

The analysis of the sufficient condition for instability is conducted on the eigenvalue spectrum. As mentioned above, when the algorithm used to compute the steady sliding solution was presented, those solutions were computed and analyzed here for a set of friction coefficient values because the friction coefficient plays a key role in the stability. The analysis of the sufficient conditions is conducted as follows :

\section{- Divergence instability}

It would be characterized by the existence of a real positive eigenvalue. In the various examples, divergence was never obtained.

\section{- Flutter instability}

Because of the form of the generalized eigenvalue problem (135) (and the formulation with $\lambda^{2}$ ), there always exists complex eigenvalues with a positive real part. The possibility of flutter instability occurring is analyzed relatively to the modes and the eigenvalues. The frequency of the flutter oscillations is given by the imaginary part of the eigenvalue, and the growth rate by its real part. Flutter instability may occur if the mode and the frequency are low enough (for energetic reasons) and if the growth rate is large enough. This will be discussed on the various examples given below.

\section{b - Analysis of the flutter instability on a simplified waist seal geometry (see Fig. 18)}

The eigenvalue spectrum is given in Fig. 39 with friction coefficient values ranging from 0 to 1.2. In order to analyze the problem more closely, the growth rate corresponding to $\mu=0.5$ and $\mu=0.9$ are given in Fig. 40 and 41 .

Considering the lowest mode, it can be noted that :

- at $\mu=0.05$, this mode is a high mode, with a frequency of approximatively $4000 \mathrm{~Hz}$ and with a small growth rate,

- at $\mu=0.45$ and 0.5 , the two lowest modes are again high modes, one corresponding to a frequency of approximatively $6000 \mathrm{~Hz}$ with a small growth rate $(\tau=0.5)$, and the other one to a frequency of approximatively $6500 \mathrm{~Hz}$ with a larger growth rate $(\tau=2.3)$ (see Fig. 40),

- between $\mu=0.8$ and $\mu=1.2$, the lowest mode is the mode 4 with a frequency of $990 \mathrm{~Hz}$ and a growth rate of $\tau=2$ (see Fig. 41).

In this case, it can be concluded that a flutter instability may occur when $\mu$ is larger than 0.8 . Mode 4 may be excited with a frequency of $990 \mathrm{~Hz}$. This mode will be more easily excited than the higher mode which occurs at $6000 \mathrm{~Hz}$ with $\mu=0.5$ although the growth rates are similar in both cases. 


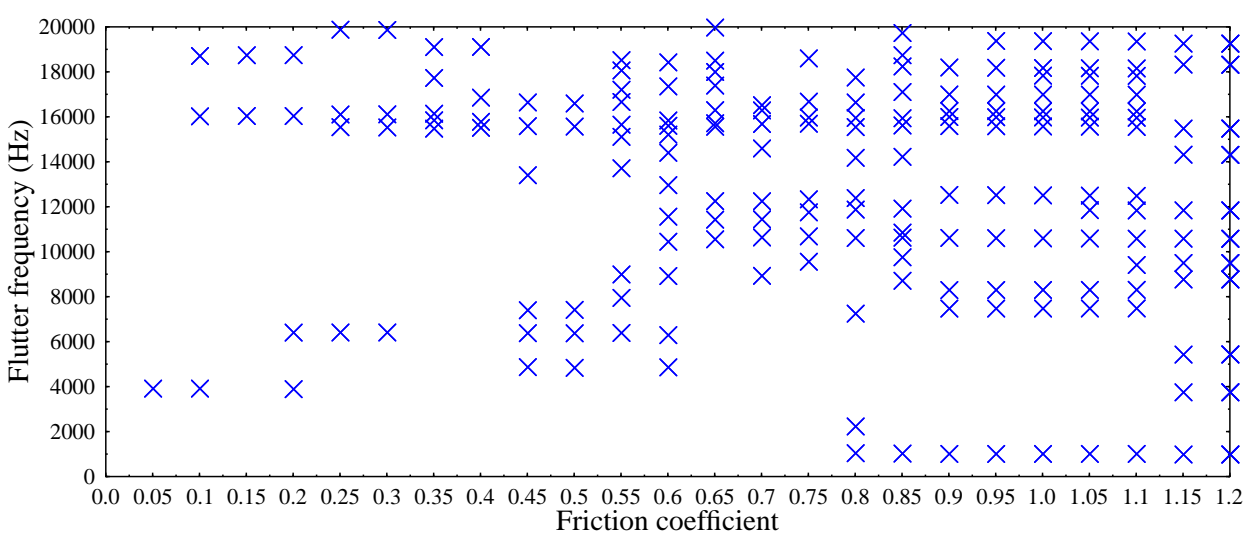

Figure 39. Flutter frequencies, $\mu=[0,1.2]$.

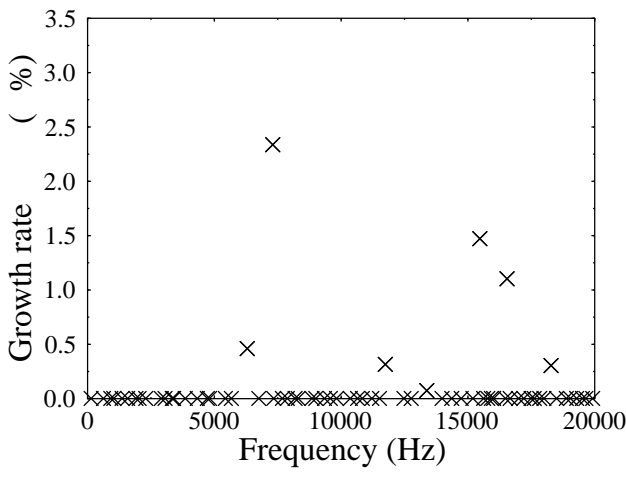

Figure 40. Eigenvalues of $\left(M^{*}\right)^{-1} K^{*}, \mu=0.5$.

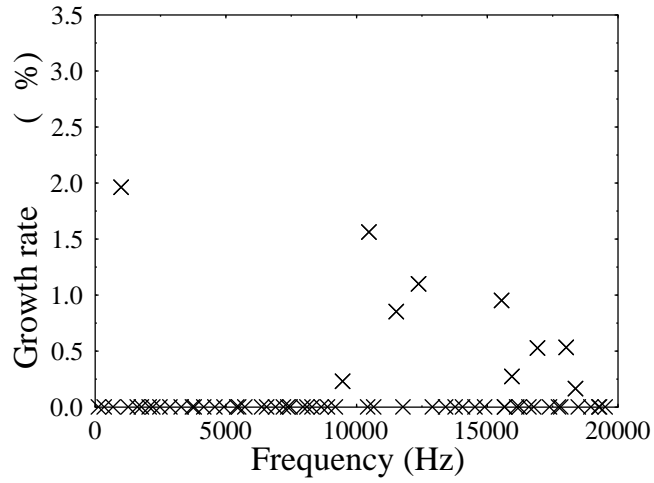

Figure 41. Eigenvalues of $\left(M^{*}\right)^{-1} K^{*}, \mu=0.9$.

\section{c - How do the eigenvalues evolve when the friction coefficient increases ?}

As shown in Fig. 42, a flutter frequency results from the coalescence of two imaginary eigenvalues. In Fig. 42, the real (growth rate) and imaginary (frequency) parts of the eigenvalues found around $1100 \mathrm{~Hz}$ have been plotted when the friction coefficient increased from 0.7 to 0.85 . It can be observed that two separate imaginary eigenvalues were obtained up to $\mu=0.7505$. These two eigenvalues then coalesce, giving two conjugate complex eigenvalues. One of them (with positive growth rate) corresponds to the possibility of flutter instability noted in Fig. 39 for $\mu=0.8$ (in fact, we give here a more accurate evaluation of the friction limit value which may give the instability : $\mu=0.7505$ ). In Fig. 42, the various steps are due to the successive loss of contact of some nodes. 

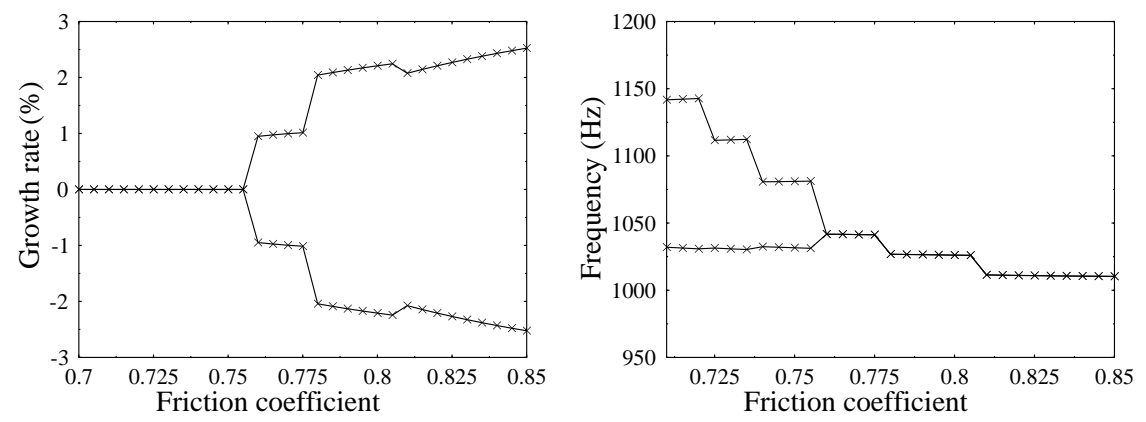

Figure 42. Coalescence of two pure imaginary eigenvalues giving a flutter instability.

\section{d - Effects of the mesh refinement}

The mesh given in Fig. 17 has been refined by cutting each element into 4 and then into 9 parts. The analysis of the sufficient conditions on the various meshes shows, as previously noted, that the refinement mainly affects the high modes in the spectrum. The mesh acts mainly as a filter : the finer it is, the higher the modes which are captured will be. Nevertheless, we are still working on this point, which is a key one in the numerical approximations.

\section{e - Effects of the compressibility}

The influence of the compressibility tolerance is an important point which needs to be checked because the stiffness matrix depends strongly on the value of coefficient $\kappa$, and this could affect the instability analysis when the eigenvalues will be computed.

The analysis shows that the effects of $\kappa$ are small as long as the incompressibility is maintained :

- the effect can be seen to be small in Fig. 43 between $\kappa=200 M P a$ and $\kappa=1200 M P a$, but in these two cases, the values are sufficiently high to ensure that the compressibility is lower than $0.01 \%$ without requiring any Lagrangian augmentations,

- its effects are again small in Fig. 44 between $\kappa=10 M P a$ and $\kappa=1200 M P a$, but here, it was necessary to perform Lagrangian augmentations for $\kappa=10 M P a$ to maintain the incompressibility,

- however, a difference can be noted in Fig. 45 between $\kappa=10 M P a$ and $\kappa=1200 \mathrm{MPa}$; here, no augmentations have been performed and incompressibility is not satisfied in the case of the solutions indicated by a circle in Fig. 45.

In conclusion, the value of $\kappa$ itself is not very decisive, but the accuracy of the incompressibility is an important factor : either a large value of $\kappa$ must be taken, or a sufficiently large number of Lagrangian augmentations must be carried out. 


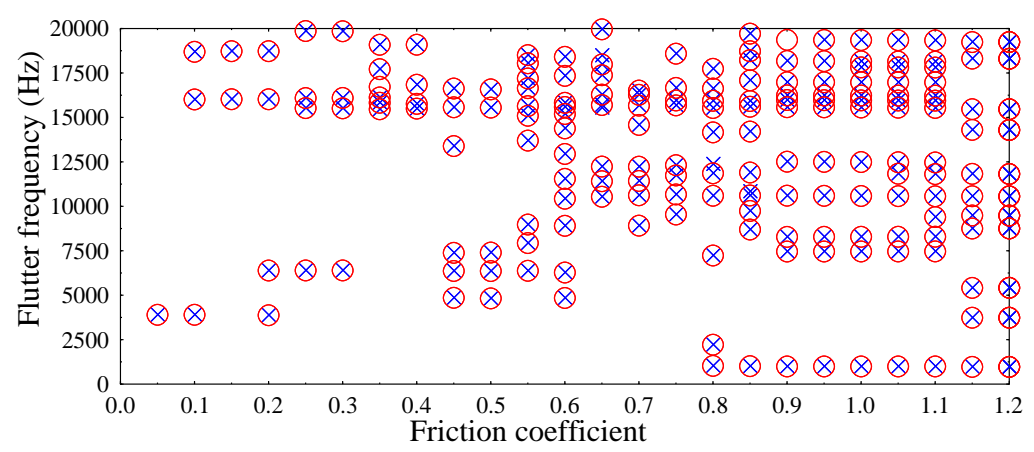

Figure 43. Comparison between the flutter frequencies obtained with $\kappa=200 M P a$ (circles) and $\kappa=1200 M P a$ (cross), $\mu=[0,1.2]$.

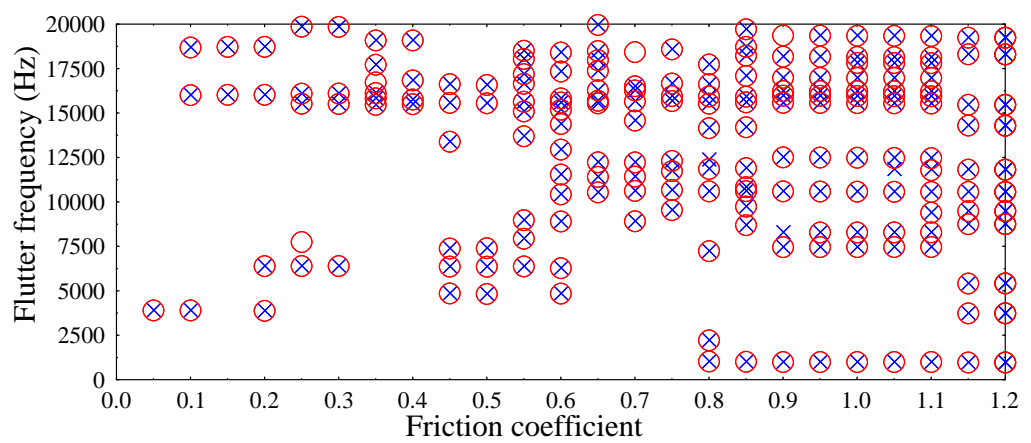

Figure 44. Comparison between the flutter frequencies obtained with $\kappa=10 M P a$ with Lagrangian augmentations (circles) and $\kappa=1200 M P a$ (cross), $\mu=[0,1.2]$.

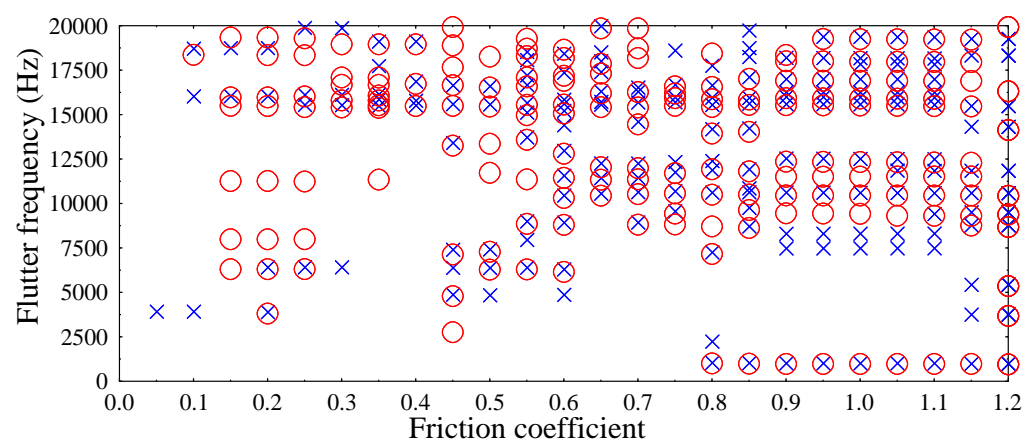

Figure 45. Comparison between the flutter frequencies obtained with $\kappa=10 M P a$ without any Lagrangian augmentations (circles) and with $\kappa=1200 M P a$ (cross), $\mu=[0,1.2]$. 


\section{f - Computation of the flutter solution}

The dynamic computation of the flutter is now carried out with the algorithm presented in Section 3. When a sufficient condition for instability is fulfilled, we compute the dynamic evolution of the solution when a small perturbation is prescribed. Due to the non smooth characteristics of the dynamics which may include normal shocks and stick-slip phenomena, the nonlinear problem is solved by using the specific numerical approach given in Section 3.

Taking the steady sliding solution as initial condition and the numerical round-off as initial perturbation, we compute then the dynamic solution. The results given in Fig. 46 were obtained with $\Delta t=10^{-4} s, T=1 s, \theta=0.55, \mu=0.9$. In Fig. 46, the solutions are given for four different initial perturbations. The first one is simply the round-off error. In the other three cases, the perturbation is taken as follows : $\epsilon v_{o b s} \Delta t\left(\operatorname{Re}\left(\mathbf{u}^{*}\right)\right)$ for the displacements, $\epsilon v_{o b s}\left(\operatorname{Re}(\lambda) \operatorname{Re}\left(\mathbf{u}^{*}\right)\right)$ for the velocities.

In two cases, $U^{*}$ is taken to be the eigenvector associated with the eigenvalue which characterizes the instability with two different values of $\epsilon(0.01$ and 0.1$)$. In the third case, $\mathbf{u}$ is a random vector and $\epsilon=0.1$.

In all the cases considered, increasing flutter was observed, with an oscillation frequency equal to $990 \mathrm{~Hz}$, which was the value predicted by the sufficient condition analysis. The flutter growth is limited by the numerical damping, depending on the choice of $\theta$ (the influence of $\theta$ will be discussed in the next section). It can be noted that the round-off error is sufficient for flutter to occur. Other choices, and especially that where the perturbation follows the direction of the eigenvector, will speed up the onset of the flutter, and save some computational time.
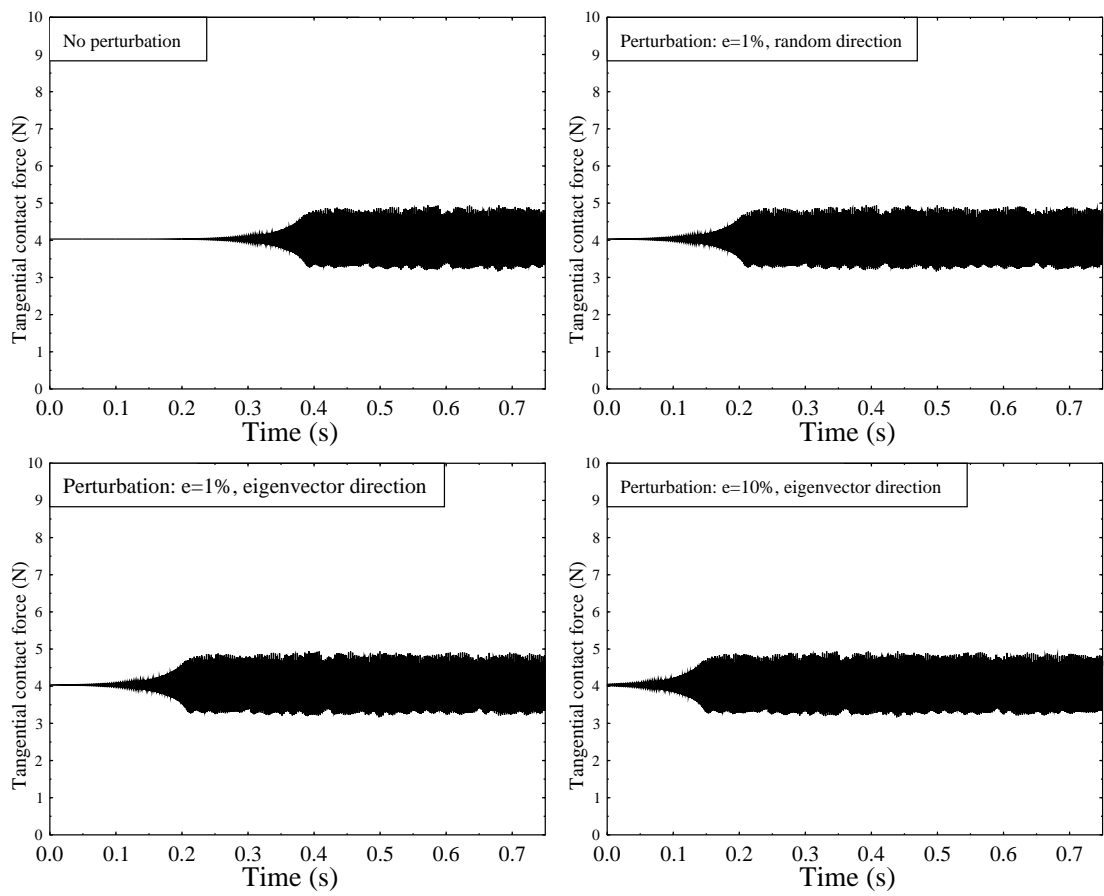

Figure 46. Effects of the magnitude of the initial perturbation. 


\subsection{Analysis of a waist seal : comparison between two geometries}

\section{a - Analysis of the flutter sufficient condition}

In this section, we propose to analyze the stability of the steady sliding solutions in the case of two kinds of waist seal sliding on a glass car window (see Vola-Raous-Martins (1999)). These steady sliding solutions were presented in Section 3 .

In Fig. 47 and 48, the total spectrum (frequency and growth rate) is given for $\mu=0.4$ in the case of the two geometries when the glass is moving down. In Fig. 49, 50, 51 and 52, the sufficient condition for flutter instability is presented along with the evolution of the lowest mode which can be excited, versus the friction coefficient values. The four figures relate geometry 1 and geometry 2, and glass moving up and glass moving down. These results show that :

- when the glass is moving up, the second geometry is more stable than the first one because a low flutter mode occurs at $\mu \geq 0.3$ in the first case, and only at $\mu \geq 0.45$ in the second one; in addition, this result is reinforced by the fact that the frequency is about $2000 \mathrm{~Hz}$ in the first case and about $6000 \mathrm{~Hz}$ in the second one,

- when the glass is moving down, the conclusion is similar but more details have to be given. As a matter of fact, from Fig. 51 and 52, it can be noted that a low flutter mode (at $3250 \mathrm{~Hz}$ in the first case and at about $3000 \mathrm{~Hz}$ in the second one) is obtained in both cases at similar values of the friction coefficient ( $\mu \geq 0.3$ in the first case and $\mu \geq 0.35$ in the second one). But, it can be checked from Fig. 47 and 48 that the corresponding growth rates differ between these two cases : at $\mu=0.4$, the growth rate is $\tau=2.4$ with geometry 1 , and $\tau=0.4$ with geometry 2. Again, the second geometry is more stable.

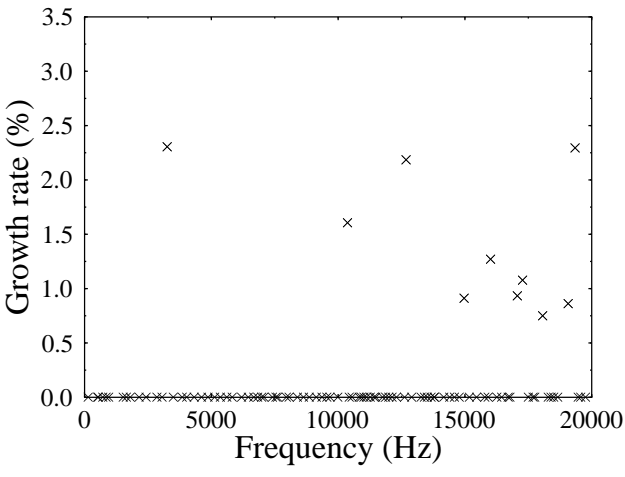

Figure 47. Eigenvalues of $M^{*-1} K^{*}$, geometry 1, $\mu=0.4$

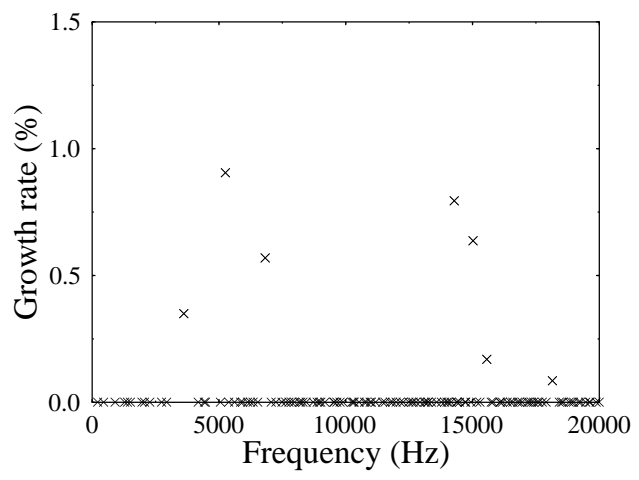

Figure 48. Eigenvalues of $M^{*-1} K^{*}$, geometry 2, $\mu=0.4$. 


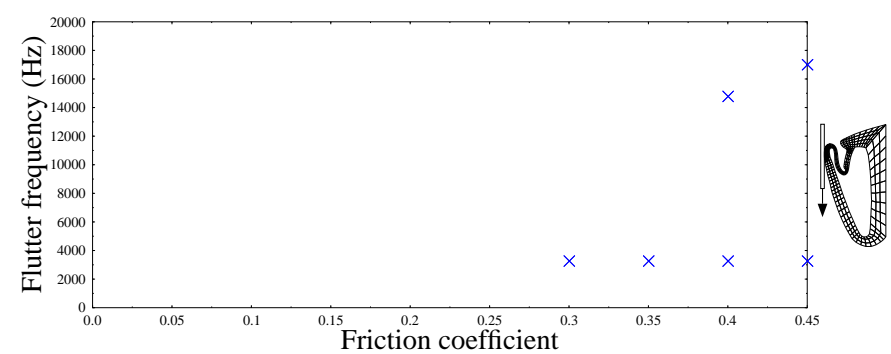

Figure 49. Flutter frequencies (geometry 1 , glass moving down, $\mu=[0,0.45]$ ).

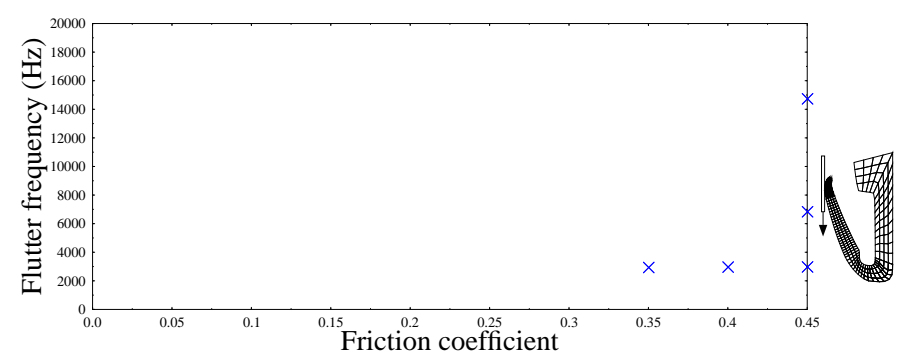

Figure 50. Flutter frequencies (geometry 2, glass moving down, $\mu=[0,0.45]$ ).

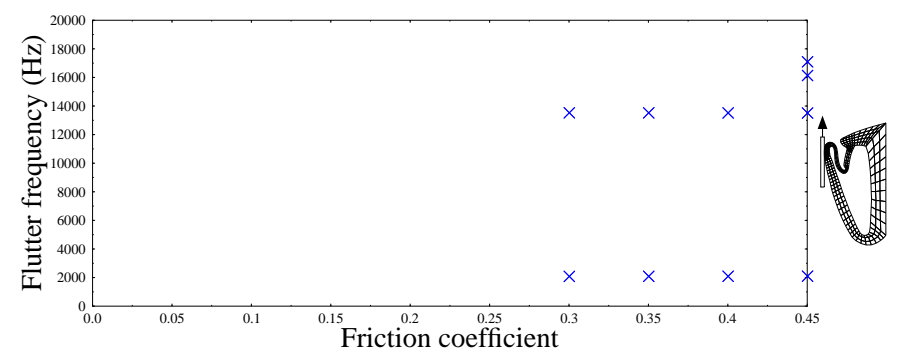

Figure 51. Flutter frequencies (geometry 1 , glass moving up, $\mu=[0,0.45]$ ).

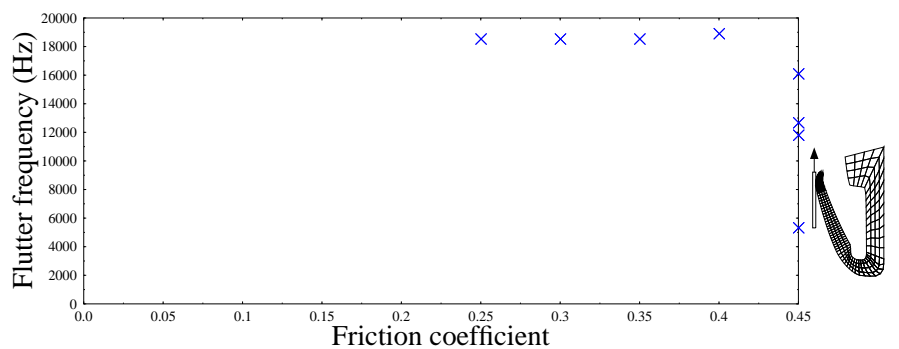

Figure 52. Flutter frequencies (geometry 2 , glass moving up, $\mu=[0,0.45]$ ). 


\section{b - Dynamic computation of the flutter}

The dynamic computation of the flutter is now carried out using the dynamic formulation presented in Section 3. This enables us to confirm the results given by the sufficient conditions of the stability analysis and to describe completely the behaviour of the structure during the flutter (normal shocks and stick-slip).

In Fig. 53 and 54, flutter is computed in the case of the two geometries with $\mu=0.4$ when the glass is moving down. In Fig. 53, the occurrence of flutter is confirmed and the predicted frequency $(3250 \mathrm{~Hz})$ is obtained. In Fig. 54, in line with our comment on the low value of the growth rate $\tau=0.4$, no flutter was observed.

Fig. 55 and 56 illustrate the influence of the numerical damping. When the numerical damping increases $(\theta=0.55$ in Fig. 55, and $\theta=0.60$ in Fig. 56), the onset of flutter is slightly delayed and the amplitude of the oscillations is reduced.

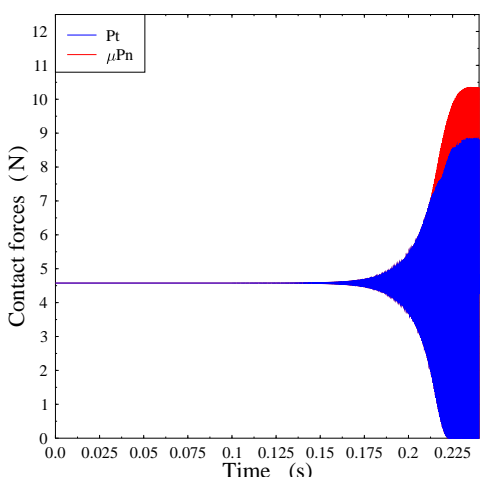

Figure 53. Evolution of the contact forces (geometry 1 , glass moving down, $\mu=0.4$, $\theta=0.55)$

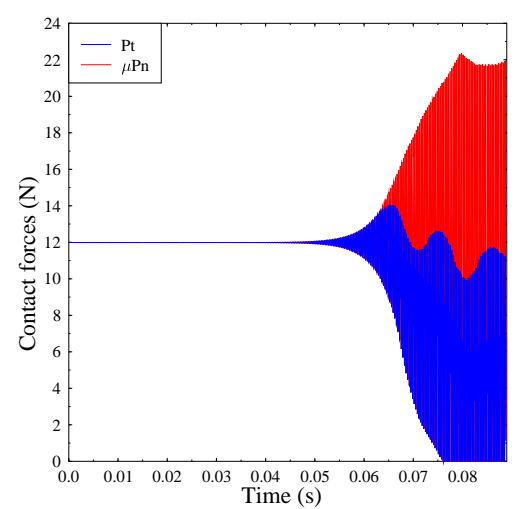

Figure 55. Evolution of the contact forces with $\mu=0.8, \theta_{1}=\theta_{2}=0.55$.

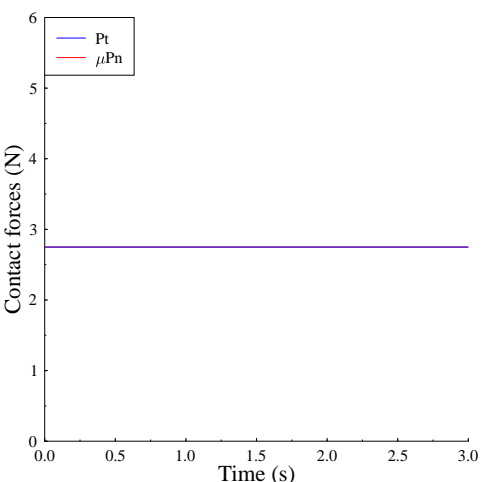

Figure 54. Evolution of the contact forces (geometry 2, glass moving down, $\mu=0.4$, $\theta=0.55)$.

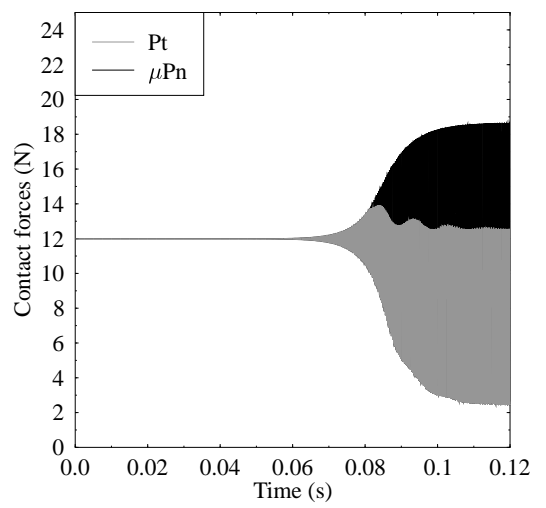

Figure 56. Evolution of the contact forces with $\mu=0.8, \theta_{1}=\theta_{2}=0.60$. 


\section{c - Analysis of the solution behaviour in the contact}

In Fig. 57 to 59, the evolution of the normal and tangential displacements is plotted using the phase plane representation $(u, \dot{u})$. In both cases, the movement converged after a while to a limit cycle.

The cycle associated with the tangential movement of node 1 (Fig. 58) reflects a stick-slip phenomenon : node 1 remains stuck during one period (the velocity of the glass is $75 \mathrm{~mm} / \mathrm{s}$ ) and slides during another one.

Fig. 59, which gives the normal movement of node 1 , shows the loss of contact and the presence of normal shocks including velocity discontinuities.

This analysis gives a good understanding of the source of the noise : the tangential and normal micro-shocks in the rubber/glass contact generate vibrations of the glass which acts as a loud speaker membrane. Computations of the eigenmodes of the glass showed that in the acoustic frequency range, the number of eigenmodes is large and there will always exist a mode which will be close enough to the excitation frequency to be triggered.

In Fig. 60, the tangential movements of all the 9 contact nodes are given with respect to time. This shows that the movement is not a global movement but that accordion effects occur in the contact : the nodes do not stick all at the same time.

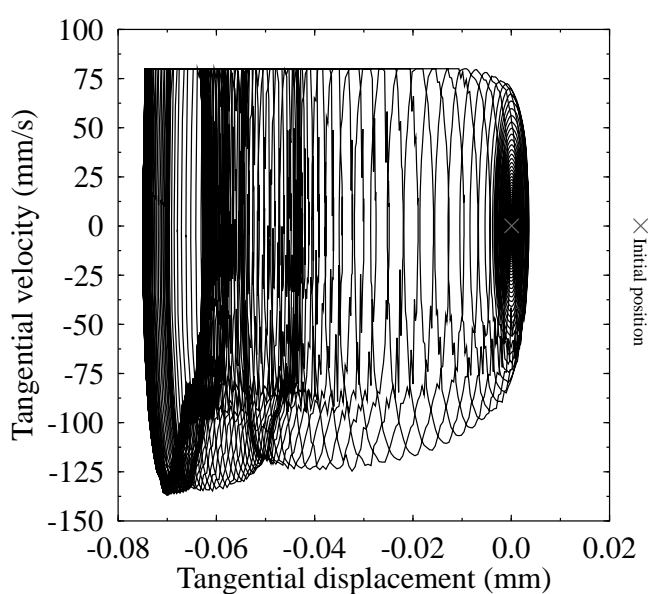

Figure 57. Tangential movement of node 1, time interval : $\tau \in[0 ; 0.25]$.

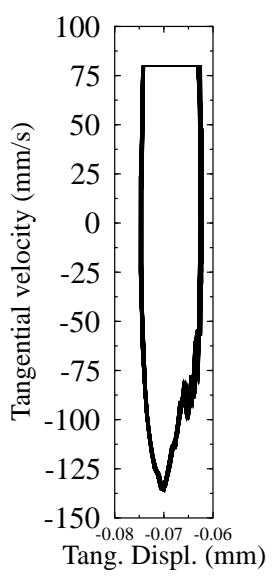

Figure 58. Limit cycle corresponding to Fig. 57, $\tau \in[0.2 ; 0.25]$. 


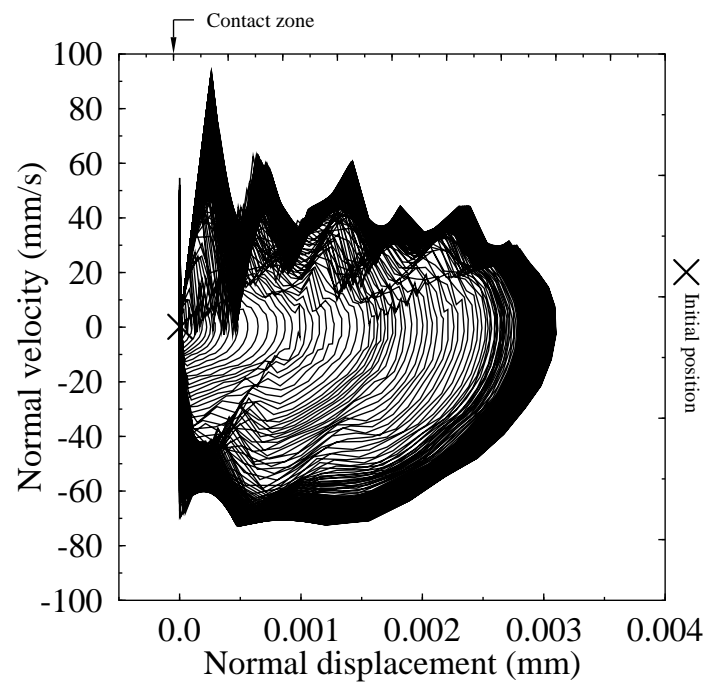

Figure 59. Normal movement of node 1 , time interval : $\tau \in[0 ; 0.25]$.

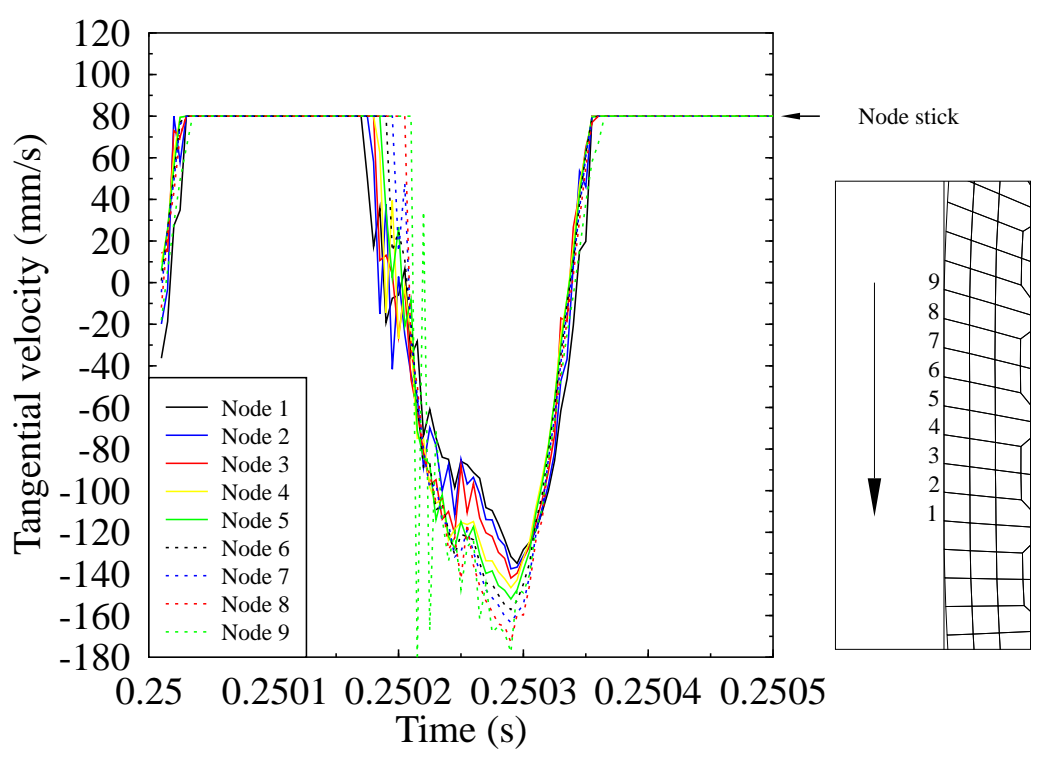

Figure 60. Evolution of the tangential velocity of the contact nodes with $\tau \in$ $[0.25 \mathrm{~s} ; 0.2505 \mathrm{~s}]$, the window velocity is of $80 \mathrm{~mm} / \mathrm{s}$. 


\section{References}

S. Barbarin (1997) Instabilité et frottement en élasticité linéaire. Application à un problème d'ondes de contrainte, Thesis, Université de Provence, Marseille.

G. Björkman, A. Klarbring, B. Sjödin, T. Larsson and M. Rönnqvist (1995). Sequential quadratic programming for non-linear elastic contact problems, Int. J. Numer. Meth. Engng., 38, 137-165.

P. Chabrand, F. Dubois and M. Raous (1998). Various numerical methods for solving unilateral contact problems with friction, Mathl. Comput. Modelling., 28, 97-108.

X. Chateau and Q.S. Nguyen (1991). Buckling of elastic structures in unilateral contact with or without friction, Eur. J. Mech., A/Solids, 10, 71-89.

R.W. Clough and J. Penzien (1975). Dynamics of structures, Int. Stud Eds, Mc Graw Hill, Kogakuska.

M. Cocu, E. Pratt and M. Raous (1996). Formulation and approximation of quasistatic frictional contact, Int. J. Engng. Sci., 34, 7, 783-798.

R. W. Cottle, J. S. Pang and R. Stone (1992). The linear complementarity problem, Computer Science and Scientific Computing, Academic press, New-York.

A. Curnier and P. Alart (1988). A generalized Newton method for contact problems with friction, J. Méca. Th. Appl., 7, 67-82.

B. Feeny, A. Guran, N. Hinrichs and K. Popp (1998). A historical review on dry friction and stick-slip phenomena, Appl. Mech. Rev., 51, 321-341.

M. S. Gadala (1986). Numerical solutions of nonlinear problems of continua - II. A survey of incompressibility constraints and software aspects, Comput. Struct., 22, 841-855.

Q. C. He, J. J. Telega and A. Curnier (1996). Unilateral contact of two solids subject to large deformations: formulation and existence results, Proc. R. Soc. Lond. A, 452, 2691-2717.

R.A. Ibrahim (1994). Friction-induced vibration, chatter, squeal and chaos: Part I - Mechanics of friction, Part II - Dynamics and modeling, Appl. Mech. Rev, 47, 209-253.

G. Isac (1992). Complementarity problems, Lecture notes in Mathematics, 1528, Springer Verlag.

M. Jean and J.J. Moreau (1987). Dynamics in the presence of unilateral contact and dry friction: a numerical approach, in Del Piero and Maceri (Eds.), Unilateral problems in structural analysis - 2, CISM lectures vol. 304, Springer Verlag, 151-196.

M. Jean (1999). The non smooth contact dynamics method, Comput. Meth. Appl. Mech. Engng.,177, 235257.

N. Josephy (1979). Newton's method for generalized equations, Report TSR 1965, Mathematics research center, University of Wisconsin.

A. Klarbring (1988). On discrete and discretized non-linear elastic structures in unilateral contact (stability, uniqueness and variational principles), Int. J. Solids Structures, 24, 459-479.

A. Klarbring (1990). Derivation and analysis of rate boundary-value problems of frictional contact, Eur. J. Mech., A/Solids, 9, 53-85.

A. Klarbring and G. Björkmann (1992). Solution of large displacement contact problems with friction using Newton's method for generalized equations, Int. J. Numer. Meth. Engng., 34, 249-269.

A. Klarbring (1997). Steady sliding and linear complementarity, in M. Ferris and J. C. Pang (Eds.), Complementarity and variational problems: state of the art, SIAM publication, Philadelphia, 132-147.

A. Klarbring (1999). Contact, friction, discrete mechanical structures and mathematical programming, in P. Wriggers - P. Panagiotopoulos (Eds.), New developments in contact problems, CISM Courses and Lectures, 384, Springer Verlag, 55-100.

A.M. Lang and D.A. Crolla (1991). Brake noise and vibration, the state of art, Vehicle Tribology, Tribologies series, 18, 165-173.

C.E. Lemke (1980). A survey of complementarity theory, in Cottle-Gianessi-Lions (Eds),Variational Inequalities and Complementarity Problems, John Wiley, New York, 213-235.

C. Licht, E. Pratt and M. Raous (1991). Remarks on a numerical method for unilateral contact including friction, Int. Series Num. Math., 101, 129-144. 
C. H. Liu, G. Hofstetter and H. A. Mang (1994). 3D finite element analysis of rubber-like materials at finite strains, Engng. comput., 11, 111-128.

J. A. C. Martins, J. T. Oden and F. M. F. Simões (1990). A study of static and kinetic friction, Int. J. Engng. Sci., 28, 29-92.

J. A. C. Martins, S. Barbarin, M. Raous and A. Pinto da Costa (1999). Dynamic stability of finite dimensional linearly elastic systems with unilateral contact and Coulomb friction, Comput. Meth. Appl. Mech. Engng., 177, n 3-4, 289-328.

J. A. C. Martins and A. Pinto da Costa (2000). Stability of finite dimensional systems with unilateral contact and friction: non-linear elastic behaviour and obstacle curvature, Int. Journal of Solids and Structures, 37, 2519-2564.

F. Moirot (1998). Etude de la stabilité d'un équilibre en présence de frottement de Coulomb, application au crissement des freins à disques, Ph.D. Thesis, Ecole Polytechnique, Paris, France.

J.-J. Moreau (1988a). Unilateral contact and dry friction in finite freedom dynamics, in Non Smooth Mechanics and Applications, CISM Courses and Lectures, 302 , J.-J. Moreau, P.D. Panagiotopoulos (Eds), Springer-Verlag, Wien, 1-82.

J.-J. Moreau (1988b). Bounded variation in time, In Moreau-Paniagiatopoulos-Strang (Eds), Topics in nonsmooth mechanics, Birkhauser Verlag, 1-74.

J.-J. Moreau (1994). Some numerical methods in multibody dynamics : application to granular materials, Eur. J. Mech. A/Solids, 13(4), 93-114.

Q.S. NGuyen (1994). Bifurcation and stability in dissipative media (plasticity, friction, fracture), Appl. Mech. Rev., 47, 1-31.

J.T. Oden and N. Kikuchi (1982). Finite element methods for constrained problems in elasticity, Int. J. Num. Meth. Eng., 19, 701-725.

J. T. Oden and J. A. C. Martins (1985). Models and computational methods for dynamic friction phenomena, Comput. Meth. Appl. Mech. Engng., 52, 527-634.

R.W. Ogden (1976). Volume change associated with the deformation of rubber-like solids, J. Mech. Phys. Solids, 24, 323-338.

S. Pandit and S. Deo (1982). Differential systems involving impulses, Lectures notes in mathematics, Springer Verlag.

A.M.F. Pinto da Costa (2001). Instabilidade e bifurcaçaões em sistemas de comportamento não-suave, Thesis, Istituto Supérior Tecnico, Lisbon, Portugal.

E. B. Pires and L. Trabucho (1990). The steady sliding problem with nonlocal friction, Int. J. Engng. Sci., 28, 631-641.

P. Rabier, J. A. C. Martins, J. T. Oden and L. Campos (1986). Existence and local uniqueness of solutions for contact problems with non-linear friction laws, Int. J. Engng. Sci., 24, 1755-1768.

C. Rajakumar and C. Rogers (1991). The Lanczos algorithm applied to unsymmetric generalized eigenvalue problem, Int. J. Numer. Meth. Engng., 32, 1009-1026.

A. Ralston and P. Rabinowitz (1978). A first course in numerical analysis, McGRAW-HILL, New-York.

M. Raous (Ed) (1988). Numerical methods in mechanics of contact involving friction, J. de Mécanique Théorique et Appliquée, Special Issue, Supp. 1 to vol. 7, Gauthier-Villars.

M. Raous, P. Chabrand and F. Lebon (1988). Numerical methods for solving unilateral contact problems with friction, in [Raous (Ed.), 1988], 111-128.

M. Raous and S. Barbarin (1992). Conjugate Gradient for Frictional Contact, In Curnier A.(Ed),Proceedings of Contact Mechanics International Symposium, Presses Polytech. et Univ. Romandes, Lausanne, 423432.

M. Raous, J.-J. Moreau and M. Jean (Eds) (1995). Contact Mechanics, Plenum Publisher, New York.

M. Raous, S. Barbarin, D. Vola and J.A.C. Martins (1995). Friction induced instabilities and sound generation, Proceed. ASME Design Engineering Technical Conference, 18-21 september 1995, Boston, USA, 799-802. 
M. Raous and S. Barbarin (1996). Stress waves in a sliding contact. Part 2 : modelling, in D. Dowson et al. (Eds.), Proceedings of the $22^{\text {nd }}$ Leeds Lyon Symposium on Tribology, Elsevier Science, 39-44.

M. Raous (1999). Quasistatic Signorini problem with Coulomb friction and coupling to adhesion, in P. Wriggers - P. Panagiotopoulos (Eds.), New developments in contact problems, CISM Courses and Lectures, 384, Springer Verlag, 101-178.

M. Raous (2001), Constitutive models and numerical methods for frictional contact, In J.Lemaitre (Ed.), Handbook of material behavior - Non linear models and properties, Section 8.5, Academic Press, 777786.

R. T. Rockafellar (1970). Convex analysis, Princeton University Press.

J. C. Simo and R. L. Taylor (1991). Quasi-incompressible finite elasticity in principal stretches. Continuum basis and numerical algorithms, Comput. Meth. Appl. Mech. Engng., 85, 272-310.

T. Sussman and K.J. Bathe (1987). A finite element formulation for nonlinear incompressible elastic and inelastic analysis, Comp. Stryct., 26, 1-2.

D. Vola (1998). Frottement et instabilités en dynamique : bruit de crissement, thesis, Université de la Méditerranée, Marseille, France.

D. Vola, E. Pratt, M. Jean and M. Raous (1998). Consistent time discretization for a dynamical frictional contact problem and complementarity techniques, Revue Européenne des Eléments Finis, 7, 149-162.

D. Vola, A. Pinto da Costa, S. Barbarin, J.A.C. Martins and M. Raous (1999). Bifurcations and instabilities in some finite dimensional frictional contact problems, in F. Pfeiffer, Ph. Glocker (Eds), Proceedings of 1998 IUTAM symposium: unilateral multibody dynamics, Kluwer, 179-190.

D. Vola, A. M. Raous and J.A.C. Martins (1999). Friction and instability of steady sliding : squeal of a rubber/glass contact, International Journal for Numerical Methods in Engineering, 46, 1699-1720.

P. Wriggers, T. Vu Van and E. Stein (1990). Finite element formulation for large deformation impact-contact problems with friction, Comp. Struct., 37 (3), 319-331.

T. Zeghloul and B. Villechaise (1996). Stress waves in a sliding contact. Part 1 : experimental study, in D. Dowson et al. (Eds.), Proceedings of the $22^{\text {nd }}$ Leeds Lyon Symposium on Tribology, Elsevier Science, 33-37. 